\title{
Silylstannylation of Allenes and Silylstannylation-Cyclization of Allenynes. Synthesis of Highly Functionalized Allyl Stannanes, Carbocyclic and Heterocyclic Compounds
}

\section{SUPPORTING INFORMATION}

\section{Table of Contents}

General Methods $\quad 3$

Synthesis of Reagents 3

Synthesis of Allenyne 1a 4

Synthesis of Allenyne 1b. 5

Synthesis of Allenyne 2 . 5

$\begin{array}{ll}\text { Synthesis of Allenyene } 3 & 6\end{array}$

$\begin{array}{ll}\text { Synthesis of Allenyne } 5 \text { (Scheme 3). } & 6\end{array}$

$\begin{array}{ll}\text { Synthesis of Allenyne } 6 \text { (Scheme 4) } & 7\end{array}$

Reduction of lactam $\mathbf{7}$ by $\mathrm{LiAlH}_{4}$ to $\mathbf{2 0}$ and $\mathbf{2 1}$ (Scheme 5). 8

Synthesis of Allenyne 8a (Scheme 6). 9

$\begin{array}{ll}\text { Synthesis of eneallene 8b (Scheme 6) } & 10\end{array}$

$\begin{array}{ll}\text { Silylstannylation-Cyclization Reactions (Table 1) } & 11\end{array}$

Reaction of 1a with trimethylsilyltributylstannane. Isolation of $\mathbf{2 5}$ (Entry 3, Table 1) 11

Conversion of $\mathbf{2 5}$ to $\mathbf{2 4}$ (Entry 2, Table 1) 11

Reaction of $\mathbf{1 b}$ with $\mathrm{Ph}_{3} \mathrm{Sn}-\mathrm{SiMe}_{2} \mathrm{Bu}^{\mathrm{t}}$ and $\mathrm{Bu}_{3} \mathrm{Sn}-\mathrm{SiMe}_{3}$ (Entry 11 Table 1 and eq 8). 12

Cyclization of Substrates 5 (Entry 4, Table 1) 12

Cyclization of Substrates $\mathbf{3}$ (Entry 5, Table 1) 13

Cyclization of Substrates 3 (Entry 6, Table 1) 13

Reactions of Cyclization Products with Electrophilic Reagents $\left(\mathrm{Br}_{2}\right.$ and $\left.\mathrm{H}^{+}\right)$.

Cyclization of Substrates 5 (Entry 8, Table 1). 14

Cyclization of Substrates 6 (Entry 9, Table 1). 15

Reaction of allenyne 5 with $\mathrm{Me}_{3} \mathrm{SiSnBu}_{3}$ at rt (Entry 5, Table 4, Eq 10). 15 
Addition of a borostannane to 1a (Entry 1, Table 3) 16

Addition of Distannane to Allenyne 1a (Entries 2/3, Table 3). 16

Addition of Hexamethylditin to $\mathbf{3}$ (Entry 4, Table 3). 17

Reaction of Allenyne 7 with Di-tin Reagents under Different Conditions 18

Reaction of Allenyne 7 with Tri-n-butyltinhydride. (Eq 13) 18

Synthesis of $\mathbf{4 8}$ and Addition of Hexamethylditin to $\mathbf{4 8}$ (Entry 6, Table 3, Eq 14). 18

Conversion of 1a to an acyclic allylstannanes 25 19

Reaction of 7 with $\mathrm{Ph}_{3} \mathrm{Sn}-\mathrm{SiMe}_{3}$ and n- $\mathrm{Bu}_{3} \mathrm{Sn}-\mathrm{SiMe}_{3} \quad 20$

Addition of $\mathrm{Bu}_{3} \mathrm{Sn}_{-} \mathrm{SiMe}_{3}$ to $\mathbf{4 8}$ (Entry 8, Table 4) 20

Synthesis of $\mathbf{5 5}$ (Scheme 9) 20

Addition of $\mathrm{Bu}_{3} \mathrm{Sn}_{-} \mathrm{SiMe}_{3}$ to 55 (Entry 10, Table 4) 21

Starting material 60 for Entry 11, Table 4 (Scheme 9) 21

Addition of $\mathrm{Bu}_{3} \mathrm{Sn}_{-\mathrm{SiMe}_{3}}$ to $\mathbf{6 0}$ (Entry 11, Table 4) 22

Reaction of [-lactam 8a with trimethylsilyltributylstannane (Entry12, Table 4) 23

Reaction of [-lactam 8b with trimethylsilyltributylstannane (Entry12, Table 4) 24

Cyclization of 61. Synthesis of Piperidines (Eq 17) 24

Table. Table of Chemical Shifts and Assignments of ${ }^{1} \mathrm{H}$ NMR of 64

Figure 1. nOe's of selected compounds 27

References $\quad 28$

$\begin{array}{ll}\text { NMR Spectra of selected compounds } & 29\end{array}$ 


\section{Silylstannylation of Allenes and Silylstannylation-Cyclization of Allenynes. Synthesis of Highly Functionalized Allyl Stannanes, Carbocyclic and Heterocyclic Compounds}

General Methods. Reactions were carried out in oven- or flame-dried glassware under a nitrogen atmosphere, unless otherwise noted. All solvents used were reagent grade. Diethyl ether, hexanes and tetrahydofuran (THF) were freshly distilled from sodium/benzophenone. Dichloromethane and toluene were freshly distilled from calcium hydride. Triethylamine was distilled from calcium hydride and stored over potassium hydroxide. Acetonitrile was dried over silica, distilled from calcium hydride and stored over 4 Å molecular sieves. Pyridine was distilled and stored over potassium hydroxide. Acetic anhydride was azeotroped with toluene, distilled and stored over $4 \AA$ molecular sieves. Anhydrous $N, N$-dimethylformamide was purchased from Fischer and used without purification. Except as otherwise indicated, all reactions were magnetically stirred and monitored by thin layer chromatography on E. Merck precoated $(0.25 \mathrm{~mm})$ silica gel $60 \mathrm{~F}_{254}$ plates. Flash chromatography was conducted by using silica gel 40 (Scientific Adsorbents Incorporated, Microns Flash). Yields, unless otherwise stated, refer to chromatographically and spectroscopically pure compounds. Melting points were determined on a Thomas Hoover uni-melt apparatus and are uncorrected. Chemical shift are reported relative to chloroform as standard at $\square=7.26$ for ${ }^{1} \mathrm{H}$ and $\square$ $=77.36$ for ${ }^{13} \mathrm{C}$. Coupling constants are reported in Hertz $(\mathrm{Hz})$. Compounds for which exact mass is reported exhibited no significant $\mathrm{m} / \mathrm{z}$ greater than the one of the parent peak.

Synthesis of Reagents. The synthesis of the silylstannane and borostannan reagents have been described before.

$\stackrel{\text { I }}{\mathrm{N}}$ Triethylamine $(27.9 \mathrm{~mL}, 200 \mathrm{mmol})$ was dissolved in $65 \mathrm{~mL}$ of dry hexane and the solution
$\left.\mathrm{N}_{\mathrm{N}}^{\mathrm{C}}\right]$ was cooled in an ice-bath. Boron trichloride $(100 \mathrm{~mL}$ of $1.0 \mathrm{M}$ solution in hex, $100 \mathrm{mmol})$ was added slowly with vigorous stirring, when a white precipitate came out. The mixture was warmed to RT and solution of $N, N$-dimethylethylenediamine (10.4 mL, $100 \mathrm{mmol})$ in $45 \mathrm{~mL}$ hexane was added slowly. The resulting mixture was refluxed $3 \mathrm{~h}$ and was filtered. The solid was washed with dry hexane and the solvent was stripped off the combined filtrate. The residue was distilled under reduced pressure $\left(42{ }^{\circ} \mathrm{C}, 11 \mathrm{mmHg}\right.$, lit. $72{ }^{\circ} \mathrm{C} .45 \mathrm{mmHg}$ ) to get $\sim 8.0 \mathrm{~g}$ of yellowish liquid. The product obtained releases fumes when exposed to atmosphere. A portion was 
redistilled to get $2.57 \mathrm{~g}(20 \%)$ of pure material. ${ }^{1} \mathrm{H} \mathrm{NMR}\left(400 \mathrm{MHz}, \mathrm{CDCl}_{3}\right): \square 2.63(\mathrm{~s}, 6 \mathrm{H}), 3.20$ (s, 4H); ${ }^{13} \mathrm{C} \mathrm{NMR}\left(100 \mathrm{MHz}, \mathrm{CDCl}_{3}\right)$ : $\square 33.4\left(\mathrm{CH}_{3}\right), 50.6\left(\mathrm{CH}_{2}\right) ;{ }^{11} \mathrm{~B} \mathrm{NMR}\left(128 \mathrm{MHz}, \mathrm{CDCl}_{3}\right)$ : $\square 27.0$.

$\mathrm{Me}_{3} \mathrm{Sn}-\mathrm{B}^{\mathrm{N}}{ }^{\mathrm{N}}$ The borostannane was prepared according to the literature ${ }^{\text {Error! Bookmark not defined. }}$ from the boron chloride from the previous experiment in $73 \%$ yield. ${ }^{1} \mathrm{H}$ NMR $\left(400 \mathrm{MHz}, \mathrm{C}_{6} \mathrm{D}_{6}\right)$ : $\square 0.25$ (s, 9H), $2.61(\mathrm{~s}, 6 \mathrm{H}), 2.96(\mathrm{~s}, 4 \mathrm{H}) ;{ }^{13} \mathrm{C} \mathrm{NMR}\left(100 \mathrm{MHz}, \mathrm{C}_{6} \mathrm{D}_{6}\right)$ : $\square \square 11.6,35.2\left(\mathrm{CH}_{3}\right), 52.6$ $\left(\mathrm{CH}_{2}\right) ;{ }^{11} \mathrm{~B}$ NMR $\left(128 \mathrm{MHz}, \mathrm{C}_{6} \mathrm{D}_{6}\right): \square 39.4\left(J_{\mathrm{Sn}-\mathrm{B}}=446 \mathrm{~Hz}\right) ;{ }^{119} \mathrm{Sn} \mathrm{NMR}\left(186 \mathrm{MHz}, \mathrm{C}_{6} \mathrm{D}_{6}\right): \square-110.7$ $\left(J_{\mathrm{Sn}-\mathrm{B}}=2088 \mathrm{~Hz}\right)$.

Synthesis of Diethyl 2-(buta-2,3-dienyl)-2-(prop-2-ynyl)malonate (1a). To a solution of diethylpropargyl malonate $(0.704 \mathrm{~g}, 3.14 \mathrm{mmol})$ in $\mathrm{CH}_{3} \mathrm{CN}(5 \mathrm{~mL})$ was added paraformaldehyde (151 mg, $5.02 \mathrm{mmol}), \mathrm{CuBr}(225 \mathrm{mg}, 1.57 \mathrm{mmol})$, and ${ }^{\mathrm{i}} \mathrm{Pr}_{2} \mathrm{NH}(0.53 \mathrm{~mL}, 3.8 \mathrm{mmol})$ and the mixture was heated to reflux for $6 \mathrm{~h}$. The solvent was evaporated and the residue was directly loaded on a silica gel column and eluted with EtOAc:Hex (1/10) to get a colorless oil (98 mg, $23 \%)$. Trace amount of bis-allene side product (resulting from diethyl dipropargylmalonate) can be cleanly separated (Alternatively, Gore, J. et al have provided the synthesis of this compound via the Pd catalyzed addition of malonate to 2,3-butadienyl phosphonate with complete suppression of multialkylation problem. ${ }^{3}$ ). Diethyl 2,3-butadienylo malonate: ${ }^{1} \mathrm{H}$ NMR (500 $\left.\mathrm{MHz}, \mathrm{CDCl}_{3}\right)$ : $\square 1.27$ (t, J $=7.2 \mathrm{~Hz}, 6 \mathrm{H}), 2.58(\mathrm{~m}, 2 \mathrm{H}), 3.46(\mathrm{t}, \mathrm{J}=7.5 \mathrm{~Hz}, \mathrm{H}), 4.20(\mathrm{dq}, \mathrm{J}=1.2,7.2 \mathrm{~Hz}, 4 \mathrm{H}), 4.70(\mathrm{~m}, 2 \mathrm{H})$, 5.14 (quintet, $\mathrm{J}=6.7 \mathrm{~Hz}, \mathrm{H}) ;{ }^{13} \mathrm{C} \mathrm{NMR}\left(125 \mathrm{MHz}, \mathrm{CDCl}_{3}\right)$ : $\square$ 13.9, 27.2, 51.4, 61.3, 76.0, 86.6, 168.7, 208.5; GC $\left(70{ }^{\circ} \mathrm{C}-5 \min -20{ }^{\circ} \mathrm{C} / \min -250{ }^{\circ} \mathrm{C}\right): \mathrm{t}_{\mathrm{r}}=10.94 \min (97 \%)$.

To a solution of diethyl-2,3-butadienyl malonate $(180 \mathrm{mg}, 0.849 \mathrm{mmol})$ in THF $(2 \mathrm{~mL})$ was added $\mathrm{KH}$ (44.3 mg, $1.10 \mathrm{mmol}$ ) portionwise. The mixture was stirred for $30 \mathrm{~min}$ at $\mathrm{rt}$ and propargyl bromide $(0.144 \mathrm{~mL}, 1.70 \mathrm{mmol})$ was added and the resulting mixture was stirred overnight. The reaction was quenched with saturated $\mathrm{NH}_{4} \mathrm{Cl}$ solution $(3 \mathrm{~mL})$ and normal extractive workup, followed by silica gel flash chromatography $($ EtOAc/Hex $=1 / 10)$ gave $190 \mathrm{mg}(89 \%)$ of $\mathbf{1 a}$ as a colorless oil. 1a: IR (thin film) 3291, 2123, 1956, $1732 \mathrm{~cm}^{-1},{ }^{1} \mathrm{H}$ NMR (500 MHz, $\mathrm{CDCl}_{3}$ ): $\square 1.25$ $\left(\mathrm{t}, J=7.1 \mathrm{~Hz}, 6 \mathrm{H}, \mathrm{CH}_{3} \mathrm{CH}_{2} \mathrm{O}\right), 2.00\left(\mathrm{t}, J=2.7 \mathrm{~Hz}, \mathrm{H}, \mathrm{CH}_{2} \mathrm{CCH}\right), 2.77(\mathrm{td}, J=2.4,8.0 \mathrm{~Hz}, 2 \mathrm{H}$, $\left.\mathrm{CH}_{2} \mathrm{CCH}\right), 2.85\left(\mathrm{~d}, J=2.7 \mathrm{~Hz}, 2 \mathrm{H}, \mathrm{CH}_{2} \mathrm{CH}=\mathrm{C}=\mathrm{C}\right), 4.20\left(\mathrm{q}, J=7.1 \mathrm{~Hz}, 4 \mathrm{H}, \mathrm{CH}_{3} \mathrm{CH}_{2} \mathrm{O}\right), 4.67(\mathrm{td}, J$ $\left.=2.4,6.7 \mathrm{~Hz}, 2 \mathrm{H}, \mathrm{RCH}=\mathrm{C}=\mathrm{CH}_{2}\right), 4.95\left(\mathrm{tt}, J=6.7,8.0 \mathrm{~Hz}, \mathrm{H}, \mathrm{RCH}=\mathrm{C}=\mathrm{CH}_{2}\right) ;{ }^{13} \mathrm{C} \mathrm{NMR}(125 \mathrm{MHz}$, $\left.\mathrm{CDCl}_{3}\right): \square 14.4,23.0,32.0,57.4,62.1,71.8,75.1,79.2,84.2,170.0,210.6$; GC $\left(70{ }^{\circ} \mathrm{C}-5\right.$ min-20 $\left.{ }^{\circ} \mathrm{C} / \min -250{ }^{\circ} \mathrm{C}\right): \mathrm{t}_{\mathrm{r}}=12.09 \min (98 \%)$. 
Synthesis of Allenyne 1b. To a solution of diethyl 2,3-butadienyl malonate (188 mg, $0.887 \mathrm{mmol}$ ) in THF ( $3 \mathrm{~mL})$ was added KH $(39.2 \mathrm{mg}, 0.976 \mathrm{mmol})$ portionwise. The mixture was stirred for $1 \mathrm{~h}$ at $\mathrm{rt}$ and 1-bromo-2-butyne $(0.144 \mathrm{~mL}, 1.698 \mathrm{mmol})$ was added and the resulting mixture was stirred overnight. The reaction was quenched with saturated $\mathrm{NH}_{4} \mathrm{Cl}$ solution $(5 \mathrm{~mL})$ and normal extractive workup, followed by silica gel flash chromatography (EtOAc/Hex $=1 / 10)$ gave $186 \mathrm{mg}$ (79 \%) of $\mathbf{1 b}$ as a colorless oil. 1b: IR (neat): 3250, 2983, 2935, 2245, 1956, 1732, 1628, 1445, 1367, 1284, 1205, 1077, 1019, $857 \mathrm{~cm}^{-1} ;{ }^{1} \mathrm{H}$ NMR $\left(500 \mathrm{MHz}, \mathrm{CDCl}_{3}\right)$ : $\square 1.25(\mathrm{t}, J=7.0 \mathrm{~Hz}, 6 \mathrm{H}$, $\left.\mathrm{CO}_{2} \mathrm{CH}_{2} \mathrm{CH}_{3}\right), 1.74\left(\mathrm{t}, J=2.5 \mathrm{~Hz}, 3 \mathrm{H}, \mathrm{C} \equiv \mathrm{C}-\mathrm{CH}_{3}\right), 2.75\left(\mathrm{dt}, J=2.4,7.9 \mathrm{~Hz}, 2 \mathrm{H}, \mathrm{CH}_{2} \mathrm{CH}=\mathrm{C}=\mathrm{C}\right)$, $2.78\left(\mathrm{q}, J=2.5 \mathrm{~Hz}, 2 \mathrm{H}, \mathrm{CH}_{2} \mathrm{C} \equiv \mathrm{C}-\mathrm{CH}_{3}\right), 4.19\left(\mathrm{q}, J=7.0 \mathrm{~Hz}, 4 \mathrm{H}, \mathrm{CO}_{2} \mathrm{CH}_{2} \mathrm{CH}_{3}\right), 4.63-4.67(\mathrm{~m}, 2 \mathrm{H}$, $\left.\mathrm{CH}=\mathrm{C}=\mathrm{CH}_{2}\right), 4.97\left(\mathrm{~m}, \mathrm{H}, \mathrm{CH}=\mathrm{C}=\mathrm{CH}_{2}\right) ;{ }^{13} \mathrm{C} \mathrm{NMR}\left(125 \mathrm{MHz}, \mathrm{CDCl}_{3}\right)$ : $\square 3.8,14.4,23.3,32.0,57.7$, 61.8, 73.7, 74.9, 79.2, 84.5, 170.3, 210.5; HRMS calcd for $[\mathrm{M}+\mathrm{H}]$ 287.1254, found 287.1277.

Synthesis of Allenyne 2. A solution of propargyl tosylamide $(2.09 \mathrm{~g}, 10.0 \mathrm{mmol})$, paraformaldehyde (360 mg, $12.0 \mathrm{mml}), \mathrm{CuBr}(0.717 \mathrm{mg}, 5.00 \mathrm{mmol})$, and ${ }^{i} \mathrm{Pr}_{2} \mathrm{NH}(1.68 \mathrm{~mL}, 12.0$ mmol) in $\mathrm{CH}_{3} \mathrm{CN}(30 \mathrm{~mL})$ was refluxed gently for $9 \mathrm{~h}$. The crude mixture was evaporated and directly loaded on a silica gel column and was eluted with EtOAc:Hex (1/3) to get a colorless oil of $N$-tosyl- $N$-2,3-butadienylamide (0.277 g, $12 \%$ ). IR (mineral oil): 3280, 1957, $1598 \mathrm{~cm}^{-1} ;{ }^{1} \mathrm{H}$ NMR (250 MHz, $\mathrm{CDCl}_{3}$ ): $\square 2.42$ (s, 3H), 3.53-3.64 (m, 2H), 4.67 (s br, H), 4.70-4.78 (m, 2H), 4.97-5.10

$(\mathrm{m}, \mathrm{H}), 7.29(\mathrm{~d}, J=8.0 \mathrm{~Hz}, 2 \mathrm{H}), 7.75(\mathrm{~d}, J=8.0 \mathrm{~Hz}, 2 \mathrm{H}) ;{ }^{13} \mathrm{C} \mathrm{NMR}\left(63 \mathrm{MHz}, \mathrm{CDCl}_{3}\right): \square 21.9,41.9$, 78.4, 87.6, 127.6, 130.1, 137.5, 143.9, 208.4.

To a solution of 2,3-butadienyl-( $p$-toluenesulfonyl)amide ( $98 \mathrm{mg}, 0.44 \mathrm{mmol}$ ) in THF ( $1 \mathrm{~mL}$ ) was added portionwise $\mathrm{KH}$ ( $21 \mathrm{mg}, 1.2$ equiv.) and the mixture was stirred at $\mathrm{rt}$ for $1 \mathrm{~h}$. To the reaction mixture was added propargyl bromide $(0.056 \mathrm{~mL}, 0.66 \mathrm{mmol})$ and the mixture was stirred overnight. The reaction was quenched with saturated $\mathrm{NH}_{4} \mathrm{Cl}$ solution $(3 \mathrm{~mL})$ and normal extractive workup, followed by silica gel flash chromatography (EtOAc/Hex $=1 / 6)$ gave $92 \mathrm{mg}(80 \%)$ of $\mathbf{2}$ as colorless oil. 2: IR (thin film) 3288, 2120, 1955, $1598 \mathrm{~cm}^{-1}$; ${ }^{1} \mathrm{H}$ NMR (400 MHz, $\mathrm{CDCl}_{3}$ ) $\square 2.03$ (t, $J=2.5 \mathrm{~Hz}, \mathrm{H}, \mathrm{CH}_{2} \mathrm{CCH}$ ), $2.42\left(\mathrm{~s}, 3 \mathrm{H}, \mathrm{Ar}-\mathrm{CH}_{3}\right), 3.88\left(\mathrm{td}, J=2.5,7.1 \mathrm{~Hz}, 2 \mathrm{H}, \mathrm{CH}_{2} \mathrm{CH}=\mathrm{C}=\mathrm{C}\right.$ ), 4.15 (d, $J=2.5 \mathrm{~Hz}, 2 \mathrm{H}, \mathrm{CH}_{2} \mathrm{CCH}$ ), $4.78\left(\mathrm{td}, J=2.5,6.6 \mathrm{~Hz}, 2 \mathrm{H}, \mathrm{RCH}=\mathrm{C}=\mathrm{CH}_{2}\right.$ ), 5.04 (quintet, $J=6.9$ $\left.\mathrm{Hz}, \mathrm{H}, \mathrm{RC} H=\mathrm{C}=\mathrm{CH}_{2}\right), 7.29(\mathrm{~d}, J=8.0 \mathrm{~Hz}, 2 \mathrm{H}), 7.7 .(\mathrm{d}, J=8.0 \mathrm{~Hz}, 2 \mathrm{H}) ;{ }^{13} \mathrm{C} \mathrm{NMR}(100 \mathrm{MHz}$, $\left.\mathrm{CDCl}_{3}\right): \square 21.4,35.7,45.5,73.4,76.3,85.3,127.5,129.4,136.0,143.4,209.7$; HRMS calcd for $[\mathrm{M}]$ 261.0824 , found 261.0824 . 
Synthesis of Allenyene 3. To a solution of 1-phenyl-2-propyn-1-ol (1.59 g, $12.0 \mathrm{mmol})$ in THF (50 mL) was added added paraformaldehyde (722 mg, $24.1 \mathrm{mmol}), \mathrm{CuBr}(863 \mathrm{mg}, 6.02 \mathrm{mmol})$, and ${ }^{i} \operatorname{Pr}_{2} \mathrm{NH}(2.53 \mathrm{~mL}, 18.0 \mathrm{mmol})$, and the mixture was heated to reflux for $10 \mathrm{~h}$. The crude mixture was evaporated and directly loaded on a silica gel column and eluted with EtOAc:Hex (1/4, $3 \%$ EtOH) to get a colorless oil (1.08 g, $61 \%)$.

To a solution of allene from the previous step $(512 \mathrm{mg}, 3.51 \mathrm{mmol})$ in DME (13 mL) at $\mathrm{rt}$ was added, portion-wise, $\mathrm{NaH}$ (183 mg, $4.56 \mathrm{mmol}, 60 \%$ in oil), and the mixture stirred for $1 \mathrm{~h}$. To the resulting mixture was added propargyl bromide $(0.89 \mathrm{~mL}, 10.5 \mathrm{mmol})$ and the mixture was further stirred at $\mathrm{rt}$ overnight. The reaction was quenched by addition of saturated solution of $\mathrm{NH}_{4} \mathrm{Cl}(10$ $\mathrm{mL})$. Normal extractive workup, followed by silica gel flash chromatography $(\mathrm{EtOAc} / \mathrm{Hex}=1 / 10)$ gave $567 \mathrm{mg}$ (88 \%) of $\mathbf{3}$ as a colorless oil. 3: IR (thin film): 3298, 3063, 3030, 3855, 2117, 1954 $\mathrm{cm}^{-1} ;{ }^{1} \mathrm{H} \mathrm{NMR}\left(400 \mathrm{MHz}, \mathrm{CDCl}_{3}\right): \square 2.44$ (t, $\left.J=2.4 \mathrm{~Hz}, \mathrm{H}, \mathrm{CH}_{2} \mathrm{CCH}\right), 4.17(\mathrm{dd}, J=2.4,15.7 \mathrm{~Hz}$, $\left.\mathrm{H}, \mathrm{CH}_{2} \mathrm{CCH}\right), 4.25$ (dd, $\left.J=2.4,15.7 \mathrm{~Hz}, \mathrm{H}, \mathrm{CH}_{2} \mathrm{CCH}\right), 4.84(\mathrm{ddd}, J=1.5,6.6,11.2 \mathrm{~Hz}, \mathrm{H}$, $\left.\mathrm{CHCH}=\mathrm{C}=\mathrm{CH}_{2}\right), 5.14\left(\mathrm{td}, J=1.4,7.8 \mathrm{~Hz}, \mathrm{CHCH}=\mathrm{C}=\mathrm{CH}_{2}\right), 5.33(\mathrm{td}, J=6.6,7.8 \mathrm{~Hz}, \mathrm{H}, \mathrm{OCH}) ;{ }^{13} \mathrm{C}$ NMR (100 MHz, $\left.\mathrm{CDCl}_{3}\right) \square 55.3,74.3,76.6,78.5,79.5,91.9$, 126.7, 127.9, 128.3, 140.3, 208.8; HRMS calcd for [M] 184.0888, found 184.0855.

Synthesis of Allenyne 5 (Scheme 3). (a) 1-(3-Butynyl)-2,5-pyrrolidinedione (11). To a solution of mixture of 3-butyn-1-ol (764 \L, $10.1 \mathrm{mmol}, 1$ equiv.), succinimide (1 g, $10.1 \mathrm{mmol})$ and triphenylphosphine $(2.65,10.1 \mathrm{mmol}, 1$ equiv.) in $20 \mathrm{~mL}$ THF was added a solution of diethyl diazodicarboxylate $(1.59 \mathrm{~mL}, 10.1 \mathrm{mmol}, 1$ equiv.) in $10 \mathrm{~mL}$ of THF over a period of 30 minutes. The resulting solutions was stirred at room temperature for $3 \mathrm{~h}$, and the solvent was removed in vacuo. The resulting crude mixture was column chromatographed $\left(\mathrm{SiO}_{2}\right.$ : dichloromethane) to give $1.05 \mathrm{~g}(68 \%$ yield $)$ of a colorless oil. ${ }^{1} \mathrm{H} \mathrm{NMR}\left(400 \mathrm{MHz}, \mathrm{CDCl}_{3}\right): \square 3.56\left(\mathrm{~d}, \mathrm{~J}=9.5,2 \mathrm{H}, \mathrm{NCH}_{2}\right)$, $2.62\left(\mathrm{~s}, 4 \mathrm{H}, \mathrm{CCH}_{2}\right), 2.39\left(\mathrm{~m}, 2 \mathrm{H}, \mathrm{NCH}_{2} \mathrm{CH}_{2}\right), 1.88(\mathrm{t}, \mathrm{J}=2.6,1 \mathrm{H}, \mathrm{CCH}) ;{ }^{13} \mathrm{C} \mathrm{NMR}(100 \mathrm{MHz}$, $\left.\mathrm{CDCl}_{3}\right): \square 176.7,79.9,70.0,36.8,27.9,17.0$.

(b) 5-Ethoxy-1-(3-butynyl)-2-pyrrolidinone (12). To a solution of 1-(3-butynyl)-2,5-

pyrrolidinedione (400 mg, $2.57 \mathrm{mmol}$ ) in $10 \mathrm{~mL}$ of absolute ethanol cooled in an ice-water bath was added sodium borohydride (600 mg, $15.42 \mathrm{mmol}, 6$ equiv.). A 2.0 M solution of hydrogen chloride in absolute ethanol was added at a rate of 2 drops every 5 minutes over a period $2.5 \mathrm{~h}$. The reaction was then acidified with $2 \mathrm{M}$ hydrogen chloride in absolute ethanol to approximately $\mathrm{pH} 3$. The resulting mixture was stirred at $0^{\circ} \mathrm{C}$ for an additional hour and made basic by a saturated solution of 
sodium bicarbonate. The mixture was extracted 3 times by $20 \mathrm{~mL}$ of chloroform each. The organic layers were combined and washed with $10 \mathrm{~mL}$ of brine, dried $\left(\mathrm{MgSO}_{4}\right)$ and concentrated. The material was column chromatographed $\left(\mathrm{SiO}_{2}\right.$ : hexanes / ethyl acetate $\left.1 / 1\right)$ to give $240 \mathrm{mg}(50 \%$ yield) of a colorless oil. ${ }^{1} \mathrm{H}$ NMR $\left(250 \mathrm{MHz}, \mathrm{CDCl}_{3}\right)$ : $\square 5.01\left(\mathrm{~d}, \mathrm{~J}=4.8,1 \mathrm{H}, H^{5}\right), 3.52(\mathrm{~m}, 1 \mathrm{H}$, $\left.\mathrm{NCH}_{2}\right), 3.39\left(\mathrm{~m}, 2 \mathrm{H}, \mathrm{OCH}_{2}\right), 3.24\left(\mathrm{~m}, 1 \mathrm{H}, \mathrm{NCH}_{2}\right), 2.38\left(\mathrm{~m}, 2+1 \mathrm{H}, \mathrm{NCH}_{2} \mathrm{CH}_{2}+H^{4}\right), 2.21(\mathrm{~m}, 2$ $\left.\mathrm{H}, H^{4}+H^{3}\right), 1.90\left(\mathrm{t}, \mathrm{J}=2.6,1 \mathrm{H}, \mathrm{CC} H\right.$ overlapping $\left.\mathrm{m}, 1 \mathrm{H}, H^{3}\right), 1.12\left(\mathrm{t}, \mathrm{J}=7.0,3 \mathrm{H}, \mathrm{OCH}_{2} \mathrm{CH}_{3}\right)$; ${ }^{13} \mathrm{C}$ NMR (63 MHz, $\mathrm{CDCl}_{3}$ ): $\square 17487,89.5,81.4,69.5,61.5,39.2,28.6,24.6,17.7,15.1$.

(c) 5-Allenyl-1-(3-butynyl)-2-pyrrolidinone (5). A solution of 5-ethoxy-1-(3-butynyl)-2pyrrolidinone $(100 \mathrm{mg}, 0.54 \mathrm{mmol})$ in $5 \mathrm{~mL}$ of dichloromethane was treated with propargyltrimethylsilane (242 $\square \mathrm{L}, 1.62 \mathrm{mmol}, 3$ equiv.) and borontrifluoride etherate (205 $\square \mathrm{L}, 1.62$ mmol, 3 equiv.) at $0{ }^{\circ} \mathrm{C}$. The reaction was allowed to warm to room temperature over 30 minutes. The mixture was then poured onto $10 \mathrm{~mL}$ of brine. The aqueous layer was extracted three times by $10 \mathrm{~mL}$ of chloroform. The combined organic layers were washed with $5 \mathrm{~mL}$ of water, dried $\left(\mathrm{MgSO}_{4}\right)$ and concentrated. The material was column chromatographed $\left(\mathrm{SiO}_{2}\right.$ : hexanes / ethyl acetate $1 / 1)$ to give $85 \mathrm{mg}$ (88\% yield) of a colorless oil. ${ }^{1} \mathrm{H}$ NMR $\left(500 \mathrm{MHz}, \mathrm{C}_{6} \mathrm{D}_{6}\right): \square 4.57(\mathrm{~m}, 1$ $\mathrm{H}, \mathrm{CH}^{5} \mathrm{CH}$ ), $4.46\left(\mathrm{~m}, 2 \mathrm{H}, \mathrm{CHCCH}_{2}\right), 3.70$ (m overlapping quintuplet, $\mathrm{J}=7.7,1+1 \mathrm{H}, H^{5}+\mathrm{NCH}_{2}$ ), 2.97 (quintuplet, $\left.\mathrm{J}=7.1,1 \mathrm{H}, \mathrm{NCH}_{2}\right), 2.30\left(\mathrm{~m}, 1 \mathrm{H}, H^{4}\right), 2.18\left(\mathrm{~m}, 1 \mathrm{H}, H^{4}\right), 2.00(\mathrm{~m}, 1 \mathrm{H}$, $\left.\mathrm{NCH}_{2} \mathrm{CH}_{2}\right), 1.89\left(\mathrm{~m}, 1 \mathrm{H}, \mathrm{NCH}_{2} \mathrm{CH}_{2}\right), 1.72(\mathrm{t}, \mathrm{J}=2.6,1 \mathrm{H}, \mathrm{CCH}), 1.60\left(\mathrm{~m}, 1 \mathrm{H}, H^{3}\right), 1.18(\mathrm{~m}, 1 \mathrm{H}$, $\left.H^{3}\right) ;{ }^{13} \mathrm{C}$ NMR $\left(125 \mathrm{MHz}, \mathrm{C}_{6} \mathrm{D}_{6}\right): \square 208.6,173.4,91.6,81.9,76.7,70.1,57.8,39.8,29.6,26.1,17.7$; IR (film): $3308,1682 \mathrm{~cm}^{-1}$; HRMS calcd for $\mathrm{C}_{11} \mathrm{H}_{13} \mathrm{NO}:[\mathrm{M}]=175.0991$, found 175.0974 . Synthesis of Allenyne 6 (Scheme 4). (a) 1-(2,3-Butadienyl)-2,5-pyrrolidinedione (13). To a solution of mixture of 2,3-butadien-1-ol (1.10 g, 19.6 mmol, 1 equiv.), succinimide (1.94 g, 19.6 mmol) and triphenylphosphine $(5.15,19.6 \mathrm{mmol}, 1$ equiv.) in $20 \mathrm{~mL}$ THF was added a solution of diethyl azodicarboxylate (3.1 mL, $19.6 \mathrm{mmol}, 1$ equiv.) in $10 \mathrm{~mL}$ of THF over a period of 30 minutes. The resulting solutions was stirred at room temperature for $3 \mathrm{~h}$, and the solvent was removed in vacuo. The resulting crude mixture was column chromatographed $\left(\mathrm{SiO}_{2}\right.$ :

dichloromethane) to give $2.70 \mathrm{~g}$ (99\% yield) of a colorless oil. ${ }^{1} \mathrm{H}$ NMR (250 $\left.\mathrm{MHz}, \mathrm{CDCl}_{3}\right): \square 4.97$ $(\mathrm{m}, 1 \mathrm{H}, \mathrm{CCH}), 4.63\left(\mathrm{~m}, 2 \mathrm{H}, \mathrm{CCH}_{2}\right), 3.89\left(\mathrm{~m}, 2 \mathrm{H}, \mathrm{NCH}_{2}\right), 2.54\left(\mathrm{~s}, 4 \mathrm{H}, H^{3}\right) ;{ }^{13} \mathrm{C} \mathrm{NMR}(63 \mathrm{MHz}$, $\left.\mathrm{CDCl}_{3}\right): \square 207.8,176.5,85.1,77.3,36.2,27.7$.

(b) 5-Ethoxy-1-(2,3-butadienyl)-2-pyrrolidinone (14). To a solution of 1-(2,3-butadienyl)-2,5pyrrolidinedione (450 mg, $2.9 \mathrm{mmol}$ ) in $20 \mathrm{~mL}$ of absolute ethanol cooled in an ice-water bath was 
added sodium borohydride (660 mg, $17.4 \mathrm{mmol}, 6$ equiv.). A 2.0 M solution of hydrogen chloride in absolute ethanol was added at a rate of 2 drops every 5 minutes over a period $2.5 \mathrm{~h}$. The reaction was then acidified with $2 \mathrm{M}$ hydrogen chloride in absolute ethanol to approximately $\mathrm{pH} 3$. The resulting mixture was stirred at $0^{\circ} \mathrm{C}$ for an additional 30 minutes and made basic by a saturated solution of sodium bicarbonate. The mixture was extracted 3 times by $20 \mathrm{~mL}$ of chloroform each. The organic layers were combined and washed with $10 \mathrm{~mL}$ of brine, dried $\left(\mathrm{MgSO}_{4}\right)$ and concentrated. The material was column chromatographed $\left(\mathrm{SiO}_{2}\right.$ : hexanes / ethyl acetate $\left.1 / 1\right)$ to give $280 \mathrm{mg}$ (55\% yield) of a colorless oil. ${ }^{1} \mathrm{H}$ NMR (500 MHz, $\left.\mathrm{CDCl}_{3}\right)$ : $\square 5.08\left(\mathrm{~m}, 1 \mathrm{H}, H^{5}\right), 4.97(\mathrm{~m}, 1$ $\mathrm{H}, \mathrm{CCH}), 4.76\left(\mathrm{~m}, 2 \mathrm{H}, \mathrm{CCH}_{2}\right), 4.22\left(\mathrm{dm}, \mathrm{J}=14.0,1 \mathrm{H}, \mathrm{NCH}_{2}\right), 3,57$ (m, $\left.1 \mathrm{H}, \mathrm{NCH}_{2}\right), 3.46(\mathrm{q}, \mathrm{J}=$ 7.0, $\left.2 \mathrm{H}, \mathrm{OCH}_{2}\right), 2.50\left(\mathrm{~m}, 1 \mathrm{H}, H^{4}\right), 2.30\left(\mathrm{~m}, 1 \mathrm{H}, H^{4}\right), 2.03\left(\mathrm{~m}, 1 \mathrm{H}, H^{3}\right), 1.94\left(\mathrm{~m}, 1 \mathrm{H}, H^{3}\right), 1.19(\mathrm{t}, \mathrm{J}$ = 7.0, $\left.3 \mathrm{H}, \mathrm{OCH}_{2} \mathrm{CH}_{3}\right) ;{ }^{13} \mathrm{C} \mathrm{NMR}\left(125 \mathrm{MHz} \mathrm{CDCl}_{3}\right): \square 209.1,174.5,88.5,86.2,76.6,62.0,38.7$, 29.0, 25.0, 15.3.

(c) 1-(2,3-Butadienyl)-5-(2-propynyl)-2-pyrrolidinone (6). A solution of 5-ethoxy-1-(2,3butadienyl)-2-pyrrolidinone (180 $\mathrm{mg}, 0.97 \mathrm{mmol})$ in $5 \mathrm{~mL}$ of dichloromethane was treated with allenyltributylstannane (606 $\square \mathrm{L}, 2.04$ mmol, 2.1 equiv.) and trimethylsilyltriflate (335 $\square \mathrm{L}, 1.84$ mmol, 1.9 equiv.) at room temperature and stirred for 30 minutes. The reaction mixture was then poured onto $10 \mathrm{~mL}$ of brine. The aqueous layer was extracted three times by $10 \mathrm{~mL}$ of chloroform. The combined organic layers were washed with $5 \mathrm{~mL}$ of water, dried $\left(\mathrm{MgSO}_{4}\right)$ and concentrated. The material was column chromatographed $\left(\mathrm{SiO}_{2}\right.$ : hexanes / ethyl acetate $\left.1 / 1\right)$ to give $156 \mathrm{mg}(88 \%$ yield) of a colorless oil. ${ }^{1} \mathrm{H} \mathrm{NMR}\left(500 \mathrm{MHz}^{\mathrm{CDCl}}\right.$ ): $\square 5.03$ (quintuplet, $\left.\mathrm{J}=6.6,1 \mathrm{H}, \mathrm{CCH}\right), 4.76$ $\left(\mathrm{m}, 2 \mathrm{H}, \mathrm{CCH}_{2}\right), 4.27\left(\mathrm{dm}, \mathrm{J}=15.1,1 \mathrm{H}, \mathrm{NCH}_{2}\right), 3.78\left(\mathrm{~m}, 1 \mathrm{H}, H^{5}\right), 3.51(\mathrm{ddd}, \mathrm{J}=15.2,7.5,0.6,1$ $\left.\mathrm{H}, \mathrm{NCH}_{2}\right), 2.44\left(\mathrm{~m}, 2+1 \mathrm{H}, \mathrm{CH}^{5} \mathrm{CH}_{2}+H^{4}\right), 2.30\left(\mathrm{~m}, 1 \mathrm{H}, H^{4}\right), 2.16\left(\mathrm{~m}, 1 \mathrm{H}, H^{3}\right), 1.95(\mathrm{t}, \mathrm{J}=2.6,1$ $\left.\mathrm{H}, \mathrm{CH}^{5} \mathrm{CH}_{2} \mathrm{CCH}\right), 1.90\left(\mathrm{~m}, 1 \mathrm{H}, H^{3}\right) ;{ }^{13} \mathrm{C} \mathrm{NMR}\left(125 \mathrm{MHz}, \mathrm{CDCl}_{3}\right): \square 209.0,174.8,85.9,79.2,76.7$, 71.0, 55.7, 39.0, 30.0, 23.4, 23.1; IR (film): 3296, 1955, $1682 \mathrm{~cm}^{-1}$; HRMS calcd for $\mathrm{C}_{11} \mathrm{H}_{13} \mathrm{NO}$ : $[\mathrm{M}]=175.0991$, found 175.1004.

\section{Reduction of lactam 7 by $\mathrm{LiAlH}_{4}$ to Isomeric 1-(Hepta-1, 2-dien-4-yl)-2-(prop-2-}

ynyl)pyrrolidine 20 and 21 (Scheme 5). In a two-necked, round-bottomed flask equipped with a reflux condenser, rubber septum, stirring bar and a $\mathrm{N}_{2}$ inlet was placed $\mathrm{LiAlH}_{4}(1 \mathrm{mmol}, 38 \mathrm{mg})$ in 3 $\mathrm{mL}$ of ether at $0^{\circ} \mathrm{C}$. A solution of lactam $(0.53 \mathrm{mmol}, 116 \mathrm{mg})$ in $3 \mathrm{~mL}$ of ether was introduced through a syringe dropwise over a period of $10 \mathrm{~min}$. The resulting mixture was brought to room temperature and refluxed for $3.5 \mathrm{~h}$ to see a total disappearance of the starting material with the 
appearance of two non-polar spots on TLC analysis. Aqueous THF was added at $0^{\circ} \mathrm{C}$ to destroy the excess $\mathrm{LiAlH}_{4}$ and filtered through a pad of $\mathrm{MgSO}_{4}$. The $\mathrm{MgSO}_{4}$ pad was washed thoroughly with $30 \mathrm{~mL}$ of ether and the filtrate was concentrated. Purification by flash column chromatography (5, 8\% EtOAc in hexanes) afforded individual amines 20 and 21 (25 and 45 mg), and a mixture (20 $\mathrm{mg}$ ) with a combined yield of 82\%, as a light oil. Non-polar amine: ${ }^{1} \mathrm{H}$ NMR (500 MHz) $\square 5.04$ (q, $\mathrm{J}=7 \mathrm{~Hz}, 1 \mathrm{H}), 4.71-4.64(\mathrm{~m}, 2 \mathrm{H}), 3.35$ (q, J = 7.5 Hz, 1H), 2.96-2.92 (m, 1H), 2.84 (td, J = 8.5, 3

$\mathrm{Hz}, 1 \mathrm{H}), 2.54$ (q, J = 8 Hz, 1H), 2.4 (dq, J = 16, $3 \mathrm{~Hz}, 1 \mathrm{H}), 2.13$ (ddd, J = 16, 8, $1.5 \mathrm{~Hz}, 1 \mathrm{H}), 1.92$ (t, $\mathrm{J}=1.5 \mathrm{~Hz}, 1 \mathrm{H}), 1.95-1.9(\mathrm{~m}, 1 \mathrm{H}), 1.77-1.63(\mathrm{~m}, 3 \mathrm{H}), 1.59-1.34(\mathrm{~m}, 4 \mathrm{H}), 0.9(\mathrm{t}, \mathrm{J}=7 \mathrm{~Hz}, 3 \mathrm{H}) ;{ }^{13} \mathrm{C}$ NMR (125 MHz) $\square 209.1,87.2,83.2$, 74.6, 69.2, 59.2, 58.1, 46.4, 37.1, 30.9, 24.3, 22.9, 20.2, 14.3. Polar amine: ${ }^{1} \mathrm{H}$ NMR $(500 \mathrm{MHz}) \mathrm{d} 5.05$ (q, J = 7.5 Hz, 1H), 4.75-4.67 (m, 2H), 3.18-3.15 (m, 1H), 3.1-3.05 (m, 1H), 2.98-2.94 (m, 1H), 2.66 (qd, J = 9 Hz, $2.5 \mathrm{~Hz}, 1 \mathrm{H}), 2.33$ (dt, J = 16.5, 3.5 $\mathrm{Hz}, 1 \mathrm{H}), 2.13(\mathrm{ddd}, \mathrm{J}=16.5,9.5,3 \mathrm{~Hz}, 1 \mathrm{H}), 1.93$ (t, J = 1.5 Hz, 1H), 1.88-1.68 (m, 4H), 1.63-1.56 (m, 1H), 1.49-1.38 (m, 2H), 1.32-1.25 (m, 1H), 0.9 (t, J = 7 Hz, 3H); ${ }^{13} \mathrm{C}$ NMR (125 MHz) $\square 209.2$, $90.5,83.5,75.1,69.2,61.7,58.4,52,35.9,31.2,26.7,23.7,20.3,14.3$.

\section{Synthesis of Allenyne 8a (Scheme 6). (a) [3R(1'R,4R)-3-[1-(tert-Butyldimethylsilyloxy)ethyl]-} 4-(1,2-propadienyl)-azetidinone $(\mathbf{2 2})^{4}$ A solution of $\left[3 R\left(1^{\prime} R, 4 R\right)-4\right.$-acetoxy-3-[1-(tertbutyldimethylsilyloxy)ethyl]-azetidinone $(100 \mathrm{mg}, 0.35 \mathrm{mmol})$ in $5 \mathrm{~mL}$ of hexane was treated with propargyltrimethylsilane (234 $\square \mathrm{L}, 1.56 \mathrm{mmol}, 4.5$ equiv.) and borontrifluoride etherate (198 $\square \mathrm{L}$, $1.56 \mathrm{mmol}, 4.5$ equiv.) at $0{ }^{\circ} \mathrm{C}$. The reaction was allowed to warm to room temperature over 30 minutes and stirred at room temperature for $16 \mathrm{~h}$. The mixture was then poured onto $10 \mathrm{~mL}$ of brine. The aqueous layer was extracted three times by $10 \mathrm{~mL}$ of chloroform each. The combined organic layer was washed with $5 \mathrm{~mL}$ of water, dried $\left(\mathrm{MgSO}_{4}\right)$ and concentrated. The material was column-chromatographed $\left(\mathrm{SiO}_{2}\right.$ : hexanes / ethyl acetate 1/1) to give $80 \mathrm{mg}$ (85\% yield) of a white crystalline compound. m.p. $72-74^{\circ} \mathrm{C} ;{ }^{1} \mathrm{H}$ NMR (500 MHz, $\mathrm{CDCl}_{3}$ ): $\square 6.18$ (bs, $\left.1 \mathrm{H}, \mathrm{NH}\right), 5.24$ (q, J = 6.7, $\left.1 \mathrm{H}, \mathrm{CH}^{4} \mathrm{CH}\right), 4.84\left(\mathrm{~m}, 2 \mathrm{H}, \mathrm{CCH}_{2}\right), 4.17\left(2\right.$ overlapping $\left.\mathrm{m}, 1+1 \mathrm{H}, H^{3}+H^{4}\right), 2.93(\mathrm{~m}, 1 \mathrm{H}$, $\mathrm{CHOSi}), 1.16\left(\mathrm{~d}, \mathrm{~J}=6.2,3 \mathrm{H}, \mathrm{CH}_{3}\right), 0.85(\mathrm{~s}, 9 \mathrm{H}, \mathrm{Si} B u), 0.06\left(\mathrm{~s}, 6 \mathrm{H}, \mathrm{SiCH}_{3}\right) ;{ }^{13} \mathrm{C} \mathrm{NMR}(125 \mathrm{MHz}$, $\mathrm{CDCl}_{3}$ ): $\square 208.1,168.2,91.5,77.6,66.0,65.2,48.7,25.7,22.3,-5.1$; IR (film): $1756 \mathrm{~cm}^{-1}$. The material was fully characterized in the next step as allenyne 8a.

[3R(1'R,4R)-3-[1-(tert-Butyldimethylsilyloxy)ethyl]-4-(1,2-propadienyl)-1-N-(2-propynyl)azetidinone (8a). To a suspension of $\mathrm{NaH}$ (80\% in mineral oil, $12.3 \mathrm{mg}, 0.41 \mathrm{mmol}, 1.1$ equiv.) in $5 \mathrm{~mL}$ of $\mathrm{THF}$ at $0^{\circ} \mathrm{C}$ was added [3R(1'R, $\left.4 R\right)$-3-[1-(tert-butyldimethylsilyloxy)ethyl]-4-(1,2- 
propadienyl)-azetidinone 22 (100 mg, $0.37 \mathrm{mmol})$ in $2 \mathrm{~mL}$ THF over 15 minutes. The mixture was stirred at $0^{\circ} \mathrm{C}$ for an additionnal 15 minutes and was let to warm at room temperature. After 30 minutes, propargyl bromide ( $80 \%$ solution in toluene, $61 \mathrm{mg}, 0.41 \mathrm{mmol}, 1.1$ equiv.) was added dropwise. The reaction mixture was stirred at room temperature for 2 hours and then taken in 10 $\mathrm{mL}$ of ammonium chloride. The organic layer was washed with $10 \mathrm{~mL}$ of a saturated solution of sodium bicarbonate. The aqueous layer was extracted 3 times with $10 \mathrm{~mL}$ of diethyl ether each. The combined organic layers were dried $\left(\mathrm{MgSO}_{4}\right)$ and concentrated. The residue was purified by column chromatography $\left(\mathrm{SiO}_{2}\right.$ : hexanes / ethyl acetate 5/1) to give $111 \mathrm{mg}(94 \%$ yield) of a clear oil identified as 8a. ${ }^{1} \mathrm{H} \mathrm{NMR}\left(500 \mathrm{MHz}, \mathrm{CDCl}_{3}\right)$ : $\square 5.21$ (q, J = 6.7, $\left.1 \mathrm{H}, \mathrm{CH}^{4} \mathrm{CH}\right), 4.86(\mathrm{~m}, 2 \mathrm{H}$, $\left.\mathrm{CCH}_{2}\right), 4.25\left(\mathrm{dd}, \mathrm{J}=7.5,1.6,1 \mathrm{H}, H^{4}\right), 4.18\left(\mathrm{~m}, 1 \mathrm{H}, H^{3}\right)$ overlapping $4.16(\mathrm{dd}, \mathrm{J}=17.8,2.5,1 \mathrm{H}$, $\mathrm{NCH}_{2}$ ), 3.75 (dd, J = 17.8, 2.5, 1 H, NCH$\left.)_{2}\right), 2.93$ (m, $\left.1 \mathrm{H}, \mathrm{CHOSi}\right), 2.18$ (t, J = 2.5, $1 \mathrm{H}$, $\left.\mathrm{NCH}_{2} \mathrm{CCH}\right), 1.17\left(\mathrm{~d}, \mathrm{~J}=6.2,3 \mathrm{H}, \mathrm{CH}_{3}\right), 0.84(\mathrm{~s}, 9 \mathrm{H}, \mathrm{SiBu}), 0.04\left(\mathrm{~s}, 6 \mathrm{H}, \mathrm{SiCH}_{3}\right) ;{ }^{13} \mathrm{C} \mathrm{NMR}(125$ $\left.\mathrm{MHz}, \mathrm{CDCl}_{3}\right): \square 209.1,166.9,89.5,77.3,76.9,72.2,65.0,64.9,52.2,29.6,25.7,22.4,17.9$, -4.4, 5.0; IR (film): 3311, 1955, $1761 \mathrm{~cm}^{-1}$; HRMS calcd for $\mathrm{C}_{17} \mathrm{H}_{27} \mathrm{NO}_{2} \mathrm{SiNa}:[\mathrm{M}]=328.17032$, found 328.16976.

\section{Synthesis of eneallene 8b (Scheme 6). [3R(1'R,4R)-3-[1-(tert-Butyldimethylsilyloxy)ethyl]-4-} (1,2-propadienyl)-1-N-(2-propenyl)-azetidinone (8b). To a suspension of $\mathrm{NaH}(80 \%$ in mineral oil, $30 \mathrm{mg}, 1.03 \mathrm{mmol}, 1.1$ equiv.) in $5 \mathrm{~mL}$ of THF at $0^{\circ} \mathrm{C}$ was added [3R(1'R,4R)-3-[1-(tertbutyldimethylsilyloxy)ethyl]-4-(1,2-propadienyl)-azetidinone (22, $250 \mathrm{mg}, 0.93 \mathrm{mmol})$ in $2 \mathrm{~mL}$ THF over 15 minutes. The mixture was stirred at $0^{\circ} \mathrm{C}$ for an additionnal 15 minutes and was let to warm at room temperature. After 30 minutes, allyl bromide (89 $\square \mathrm{L}, 1.03 \mathrm{mmol}, 1.1$ equiv.) was added dropwise. The reaction mixture was stirred at room temperature for 2 hours and then taken in $10 \mathrm{~mL}$ of ammonium chloride. The organic layer was washed with $10 \mathrm{~mL}$ of a saturated solution of sodium bicarbonate. The aqueous layer was extracted 3 times with $10 \mathrm{~mL}$ of diethyl ether. The combined organic layers were dried $\left(\mathrm{MgSO}_{4}\right)$ and concentrated. The residue was purified by column chromatography $\left(\mathrm{SiO}_{2}\right.$ : hexanes / ethyl acetate 5/1) to give $120 \mathrm{mg}$ (42\% yield) of $\mathbf{8 b}$ as a clear oil. ${ }^{1} \mathrm{H}$ NMR (500 MHz, $\left.\mathrm{CDCl}_{3}\right)$ : $\square 5.70\left(\mathrm{q}, \mathrm{J}=6.7,1 \mathrm{H}, \mathrm{NCH}_{2} \mathrm{CH}\right), 5.18(\mathrm{dd}, \mathrm{J}=17.1,1.5,1$ $\mathrm{H}, \mathrm{NCH}_{2} \mathrm{CHCH}_{2}$ ), 5.12 (2 overlapping m, $\left.1+1 \mathrm{H}, \mathrm{NCH}_{2} \mathrm{CHCH}_{2}+\mathrm{CH}^{4} \mathrm{CH}\right), 4.80$ (dd, J = 7.0, 0.8, 2 $\left.\mathrm{H}, \mathrm{CH}^{4} \mathrm{CHCH}_{2}\right), 4.17\left(\mathrm{~m}, 1 \mathrm{H}, H^{3}\right), 4.09\left(\mathrm{dd}, \mathrm{J}=7.8,1.6,1 \mathrm{H}, H^{4}\right), 3.95(\mathrm{ddt}, \mathrm{J}=15.5,5.5,1.4,1 \mathrm{H}$, $\mathrm{NCH}_{2}$ ), 3.55 (ddd, J = 15.6, 7.0, 0.9, $\left.1 \mathrm{H}, \mathrm{NCH}_{2}\right) 2.89$ (m, $\left.1 \mathrm{H}, \mathrm{CHOSi}\right), 1.15$ (d, J = 6.3, $3 \mathrm{H}, \mathrm{CH}_{3}$ ), 0.83 (s, $9 \mathrm{H}, \mathrm{SiBu}), 0.04$ (s, $\left.3 \mathrm{H}, \mathrm{SiCH}_{3}\right), 0.02$ (s, $\left.3 \mathrm{H}, \mathrm{SiCH}_{3}\right) ;{ }^{13} \mathrm{C} \mathrm{NMR}\left(63 \mathrm{MHz}, \mathrm{CDCl}_{3}\right)$ : 
209.0, 167.2, 131.8, 118.3, 89.6, 77.0, 65.0,.64.6, 52.1, 43.1, 25.7, 22.4, 17.8, -4.5, -4.9; IR (film): 2930, 1955, $1758 \mathrm{~cm}^{-1}$; HRMS calcd for $\mathrm{C}_{17} \mathrm{H}_{29} \mathrm{NO}_{2} \mathrm{SiNa}:[\mathrm{M}]=330.18597$, found 330.18495 .

\section{Silylstannylation-Cyclization Reactions (Table 1)}

\section{Reaction of 1a with trimethylsilyltributylstannane. Isolation of the Uncyclized Allylstannanes Diethyl 2-((E)-4-(tributylstannyl)-3-(trimethylsilyl)but-2-enyl)-2-(prop-2-ynyl)malonate (25)} (Table 1, Entry 3). To a solution of $\mathrm{Pd}_{2}\left(\mathrm{dba}_{3}{ }_{3} \mathrm{CHCl}_{3}(2.6 \mathrm{mg}, 0.0050 \mathrm{mmol} \mathrm{Pd}), \mathrm{P}\left(\mathrm{C}_{6} \mathrm{~F}_{5}\right)_{3}(5.3 \mathrm{mg}\right.$, $0.0100 \mathrm{mmol}$ ), and $\mathrm{Bu}_{3} \mathrm{Sn}_{-} \mathrm{SiMe}_{3}(40.0 \mathrm{mg}, 0.110 \mathrm{mmol})$ in $1 \mathrm{~mL}$ of $\mathrm{C}_{6} \mathrm{D}_{6}$ at $\mathrm{rt}$ was added allenyne 1a $(25.0 \mathrm{mg}, 0.100 \mathrm{mmol})$ and the reaction mixture was kept in an NMR tube at $\mathrm{rt}$. Progress of the reaction was followed by ${ }^{1} \mathrm{H}$ NMR. After $2 \mathrm{~h}$ at $\mathrm{rt},{ }^{1} \mathrm{H}$ NMR spectrum indicated a clean conversion into an acyclic intermediate 25. The contents of the NMR tube was evaporated and the residual oil was purified by flash chromatography $\left(\mathrm{Hex} / \mathrm{Et}_{3} \mathrm{~N}=95 / 5\right)$ to get $51.0 \mathrm{mg}(83 \%)$ of product as a colorless oil. 25: IR (thin film): 3314, 2956, 2928, 2827, 2853, 2359, 2340, $1738 \mathrm{~cm}^{-1} ;{ }^{1} \mathrm{H}$ NMR (500 MHz, $\left.\mathrm{CDCl}_{3}\right): \square 0.02$ (s, 9H), 0.85-0.92 (m, 15H, SnBu $), 1.25$ (t, J = 7.1 Hz, 6H, $\mathrm{CH}_{3} \mathrm{CH}_{2} \mathrm{O}$ ), 1.29-1.55 (m, 12H, SnBu $), 1.91\left(\mathrm{~s}, \mathrm{~J}_{\mathrm{Sn}-\mathrm{H}}=64 \mathrm{~Hz}, 2 \mathrm{H}, \mathrm{CH}_{2} \mathrm{SnBu}_{3}\right), 1.96(\mathrm{t}, \mathrm{J}=2.6 \mathrm{~Hz}, \mathrm{H}$, $\mathrm{CH}_{2} \mathrm{CCH}$ ), 2.73 (d, J = 6.9 Hz, 2H, $\left.\mathrm{CH}_{2} \mathrm{CH}=\mathrm{CSi}\right), 2.78$ (d, J = 2.6 Hz, 2H, $\left.\mathrm{CH}_{2} \mathrm{CCH}\right), 4.19$ (dq, J = 1.3, $7.1 \mathrm{~Hz}, 4 \mathrm{H}), 5.18$ (t br, J $=6.9 \mathrm{~Hz}, \mathrm{H}, \mathrm{CH}_{2} \mathrm{CH}=\mathrm{CSi}$ ); Selected nOe data on which the assignments are based is shown in Figure 5. ${ }^{13} \mathrm{C}$ NMR $\left(125 \mathrm{MHz}, \mathrm{CDCl}_{3}\right)$ : $\square-1.2,10.5,13.4,14.1$, 14.5, 23.2, 27.8, 29.5, 31.7, 57.3, 61.9, 71.5, 80.1, 125.9, 146.3, 170.4; HRMS calcd for $\mathrm{C}_{29} \mathrm{H}_{54} \mathrm{O}_{4} \mathrm{SiSnNa}[\mathrm{M}+\mathrm{Na}]^{+}$637.2711, found 637.2719; nOe experiments confirmed the stereochemistry depicted above.

Table 1, Entry 2 (conversion of 25 to 24). To a solution of $\mathrm{Pd}_{2}(\mathrm{dba})_{3} \mathrm{CHCl}_{3}(2.6 \mathrm{mg}, 0.0050$ mmol Pd), $\mathrm{P}\left(\mathrm{C}_{6} \mathrm{~F}_{5}\right)_{3}(5.3 \mathrm{mg}, 0.0100 \mathrm{mmol})$, and $\mathrm{Bu}_{3} \mathrm{Sn}_{-}-\mathrm{SiMe}_{3}(40 \mathrm{mg}, 0.110 \mathrm{mmol})$ in $1 \mathrm{~mL}$ of $\mathrm{C}_{6} \mathrm{D}_{6}$ at $\mathrm{rt}$ was added allenyne $1 \mathrm{a}(25.0 \mathrm{mg}, 0.100 \mathrm{mmol})$ and the reaction mixture was kept in an NMR tube at rt. Progress of the reaction was followed by ${ }^{1} \mathrm{H}$ NMR. After $1.5 \mathrm{~h}$ at rt, ${ }^{1} \mathrm{H}$ NMR spectrum indicated a clean conversion into an acyclic intermediate 25 . This reaction mixture was heated at $80{ }^{\circ} \mathrm{C}$ for $8 \mathrm{~h}$. The ${ }^{1} \mathrm{H}$ NMR at this point indicates a clean conversion into a cyclic product (Z)-Diethyl 3-((tributylstannyl)methylene)-4-(1-(trimethylsilyl)vinyl)cyclopentane-1,1dicarboxylate (24) with no acyclic product remaining. The contents of the NMR tube were evaporated and the residual oil was purified by flash chromatography $\left(\mathrm{Hex} / \mathrm{Et}_{3} \mathrm{~N}=95 / 5\right)$ to get 59 
mg (92\%) of product as a colorless oil. 24. IR (film): 2956, 2926, 2872, 2854, 1732, 1615, 1463, 1376, 1368, $1249 \mathrm{~cm}^{-1}$; ${ }^{1} \mathrm{H}$ NMR (500 MHz, $\mathrm{CDCl}_{3}$ ): $\square 0.14$ (s, 9H), signals due to $\mathrm{SnBu}_{3}$ group and $\mathrm{CH}_{3} \mathrm{CH}_{2} \mathrm{O}$ are omitted, $1.99\left(\mathrm{dd}, \mathrm{J}=5.9,7.1 \mathrm{~Hz}, \mathrm{H}\right.$, ring $\left.\mathrm{CH}_{2} \mathrm{CH}\right), 2.80(\mathrm{dd}, \mathrm{J}=8.0,12.4 \mathrm{~Hz}, \mathrm{H}$, ring $\left.\mathrm{CH}_{2} \mathrm{CH}\right), 2.83\left(\mathrm{~d}, \mathrm{~J}=15.6 \mathrm{~Hz}, \mathrm{H}\right.$, ring $\left.\mathrm{CH}_{2}\right), 3.23\left(\mathrm{~d}, \mathrm{~J}=15.8 \mathrm{~Hz}, \mathrm{H}\right.$, ring $\left.\mathrm{CH}_{2}\right), 3.29(\mathrm{t} b r, \mathrm{~J}=$ $7.1 \mathrm{~Hz}, \mathrm{H}, \mathrm{CH}_{2} \mathrm{CH}$ ), 4.12-4.22 (m, 4H, $\mathrm{OCH}_{2} \mathrm{CH}_{3}$ ), 5.35 (s br, H, $\mathrm{CH}_{2}=\mathrm{CSi}$ ), 5.67 (t br, J = 1.9 Hz, $\left.\mathrm{H}, \mathrm{CH}_{2}=\mathrm{CSi}\right), 5.96\left(\mathrm{~s},{ }^{2} \mathrm{~J}_{\mathrm{Sn}-\mathrm{H}}=64 \mathrm{~Hz}, \mathrm{H}, \mathrm{CHSn}\right) ;{ }^{13} \mathrm{C} \mathrm{NMR}\left(125 \mathrm{MHz}, \mathrm{CDCl}_{3}\right)$ : $\square 0.00,10.3,14.1$, 14.4, 14.5, 27.8, 29.5, 41.3, 46.2, 50.0, 59.7, 61.8, 61.9, 123.8, 125.1, 153.9, 159.1, 171.9, 172.0;

${ }^{119} \mathrm{Sn}$ NMR (186 MHz, CDCl $)$ ]-61.4; HRMS (FAB) calcd for $\mathrm{C}_{29} \mathrm{H}_{54} \mathrm{O}_{4} \mathrm{SiSnNa}\left[(\mathrm{M}+\mathrm{Na})^{+}\right]$ 637.2717, found 637.2716.

\section{Reaction of $1 \mathrm{~b}$ with $\mathrm{Ph}_{3} \mathrm{Sn}-\mathrm{SiMe}_{2} \mathrm{Bu}^{\mathrm{t}}$ and $\mathrm{Bu}_{3} \mathrm{Sn}-\mathrm{SiMe}_{3}$ under the Standard Cyclization}

Conditions (Table 1, entry 11 and eq 8). To a solution of $\mathrm{Pd}\left(\mathrm{PhCN}_{2} \mathrm{Cl}_{2}(1.9 \mathrm{mg}, 0.0050 \mathrm{mmol}\right.$ $\mathrm{Pd}$ ), $\mathrm{P}\left(\mathrm{C}_{6} \mathrm{~F}_{5}\right)_{3}(5.3 \mathrm{mg}, 0.0100 \mathrm{mmol})$, and $\mathrm{Ph}_{3} \mathrm{Sn}_{-} \mathrm{SiMe}_{2} \mathrm{Bu}^{\mathrm{t}}(51 \mathrm{mg}, 0.110 \mathrm{mmol})$ in $1 \mathrm{~mL}$ of $\mathrm{C}_{6} \mathrm{D}_{6}$ at $\mathrm{rt}$ was added allenyne $\mathbf{1 b}(26.4 \mathrm{mg}, 0.100 \mathrm{mmol})$ and the reaction mixture was kept in an NMR tube at rt. Progress of the reaction was followed by ${ }^{1} \mathrm{H}$ NMR. After 2 days at $80^{\circ} \mathrm{C}$, starting $\mathbf{1 b}$ remained intact and even after 3 days at $80{ }^{\circ} \mathrm{C}$, starting material remained largely intact with less than $15 \%$ conversion into an unidentified mixture. The attempts to isolate the product failed.

When $\mathrm{Bu}_{3} \mathrm{Sn}-\mathrm{SiMe}_{3}$ was used instead, after 2 days at $80{ }^{\circ} \mathrm{C}$, the reaction mixture shows acyclic adduct 36 in a mixture with other side-products with no starting material remaining. An insitu ${ }^{1} \mathrm{H}$ NMR $\left(\mathrm{C}_{6} \mathrm{D}_{6}\right)$ indicated a peak at $\square 5.60(\mathrm{t}, J=7.0 \mathrm{~Hz})$ assigned to $\mathrm{C} \underline{\mathrm{H}}=\mathrm{C}\left(\mathrm{SiR}_{3}\right) \mathrm{CH}_{2}\left(\mathrm{SnR}_{3}\right)$ by analogy with the structure of 25 .

Table 1, Entry 4. To a solution of $\mathrm{Pd}_{2}(\mathrm{dba})_{3} \mathrm{CHCl}_{3}(2.6 \mathrm{mg}, 0.0050 \mathrm{mmol} \mathrm{Pd}), \mathrm{P}\left(\mathrm{C}_{6} \mathrm{~F}_{5}\right)_{3}(5.3 \mathrm{mg}$, $0.0100 \mathrm{mmol}$ ), and $\mathrm{Ph}_{3} \mathrm{Sn}-\mathrm{SiMe}_{2} \mathrm{Bu}^{\mathrm{t}}(51 \mathrm{mg}, 0.110 \mathrm{mmol})$ in $1 \mathrm{~mL}$ of $\mathrm{C}_{6} \mathrm{D}_{6}$ at $\mathrm{rt}$ was added allenyne $2(26.1 \mathrm{mg}, 0.100 \mathrm{mmol})$ and the reaction mixture was kept in an NMR tube at rt. Progress of the reaction was followed by ${ }^{1} \mathrm{H}$ NMR. After $24 \mathrm{~h}$ at $\mathrm{rt}$, a clean conversion into cyclic product 26 was observed. The contents of the NMR tube was evaporated and the residual oil was purified by flash chromatography $\left(\mathrm{EtOAc} / \mathrm{Hex}=1 / 10,3 \% \mathrm{Et}_{3} \mathrm{~N}\right)$ to get $30.0 \mathrm{mg}(41 \%)$ of product as a colorless oil. 26: IR (thin film) 3064, 2955, 2928, 2856, 1622, 1598, 1480, 1470, 1348, 1265, $1165 \mathrm{~cm}^{-1}$; ${ }^{1} \mathrm{H}$ NMR (500 MHz, $\left.\mathrm{CDCl}_{3}\right)$ : $\square-0.34$ ((s, 3H), -0.19 (s, 3H), 0.80 (s, 9H), 2.48 (s, 3H), 3.14 (dd, $J=6.6,9.1$ $\mathrm{Hz}, \mathrm{H}), 3.35(\mathrm{~d}$ br, $J=6.2 \mathrm{~Hz}, \mathrm{H}), 3.38(\mathrm{~d}, J=9.2 \mathrm{~Hz}, \mathrm{H}), 4.16(\mathrm{~d} \mathrm{br}, J=14.1 \mathrm{~Hz}, \mathrm{H}), 5.27(\mathrm{t}, J=$ $1.6 \mathrm{~Hz}, \mathrm{H}), 5.81(\mathrm{t}, J=1.8 \mathrm{~Hz}, \mathrm{H}), 6.28\left(\mathrm{~s},{ }^{2} J_{\mathrm{Sn}-\mathrm{H}}=80 \mathrm{~Hz}, \mathrm{H}\right), 7.30-7.70(\mathrm{~m}, 15 \mathrm{H}) ;{ }^{13} \mathrm{C}$ NMR $(125$ 
$\left.\mathrm{MHz}, \mathrm{CDCl}_{3}\right) \square-6.2,-4.2,16.5,21.4,50.4,53.8,54.7$; HRMS calcd for $[\mathrm{M}+\mathrm{Na}]$ 750.1860, found 750.1856; A selected nOe contacts confirmed the double bond configuration and ring structure depicted (Figure 1).

Table 1, Entry 5. To a solution of $\mathrm{Pd}(\mathrm{PhCN})_{2} \mathrm{Cl}_{2}(1.9 \mathrm{mg}, 0.0050 \mathrm{mmol} \mathrm{Pd}), \mathrm{P}\left(\mathrm{C}_{6} \mathrm{~F}_{5}\right)_{3}(5.3 \mathrm{mg}$, $0.0100 \mathrm{mmol}$ ), and $\mathrm{Ph}_{3} \mathrm{Sn}-\mathrm{SiMe}_{2} \mathrm{Bu}^{\mathrm{t}}$ (51 mg, $0.110 \mathrm{mmol}$ ) in $1 \mathrm{~mL}$ of $\mathrm{C}_{6} \mathrm{D}_{6}$ at $\mathrm{rt}$ was added allenyne 3 (18.4 $\mathrm{mg}, 0.100 \mathrm{mmol}$ ) and the reaction mixture was kept in an NMR tube at rt. Progress of the reaction was followed by ${ }^{1} \mathrm{H}$ NMR. After $2.5 \mathrm{~h}$ at $\mathrm{rt},{ }^{1} \mathrm{H}$ NMR of the mixture showed a mixture of 3 and product 27 (ratio 1:1). After $12 \mathrm{~h}$ at $\mathrm{rt}$, a clean conversion into cyclic product 27 was observed with less than $<5 \%$ of starting material remaining. Solvent was evaporated from the contents of the NMR tube and the residual oil was purified by flash chromatography $\left(\mathrm{Hex} / \mathrm{Et}_{3} \mathrm{~N}=95 / 5\right)$ followed by careful second chromatography to get $40 \mathrm{mg}(61 \%)$ of product as a colorless oil. 27: IR (thin film): 3063, 2956, 2928, 2855, 1954, 1877, 1819, 1727, 1626, 1579, 1463, 1429, $1265 \mathrm{~cm}^{-1} ;{ }^{1} \mathrm{H}$ NMR (500 MHz, $\left.\mathrm{CDCl}_{3}\right): \square-0.27$ (s, 3H, $\mathrm{SiCH}_{3}$ ), -0.14 (s, 3H, $\mathrm{SiCH}_{3}$ ), 0.89 (s, 9H, t-Bu), 3.53 (s br, $\left.\mathrm{H}, \mathrm{CH}(\mathrm{TBS}) \mathrm{C}=\mathrm{CH}_{2}\right), 4.50\left(\mathrm{dd}, J=1.6,13.5 \mathrm{~Hz}, \mathrm{H}, \mathrm{CH}_{2} \mathrm{C}=\mathrm{C}\right), 4.63(\mathrm{td}, J=1.6,13.5 \mathrm{~Hz}, \mathrm{H}$, $\left.\mathrm{CH}_{2} \mathrm{C}=\mathrm{C}\right), 5.03(\mathrm{~s}, \mathrm{H}, \mathrm{OCH}), 5.56\left(\mathrm{t}, J=1.6 \mathrm{~Hz}, \mathrm{SiC}=\mathrm{CH}_{2}\right), 5.94\left(\mathrm{t}, J=1.8 \mathrm{~Hz}, \mathrm{H}, \mathrm{SiC}=\mathrm{CH}_{2}\right), 6.24$ $\left(\mathrm{s},{ }^{2} J_{\mathrm{Sn}-\mathrm{H}}=74 \mathrm{~Hz}, \mathrm{H}, \mathrm{CHSn}\right), 7.24-7.40(\mathrm{~m}, 5 \mathrm{H}) ;{ }^{13} \mathrm{C} \mathrm{NMR}\left(125 \mathrm{MHz}, \mathrm{CDCl}_{3}\right): \square-5.0,-3.0,17.6$, 27.5, 58.4, 73.7, 85.5, 117.4, 126.2, 127.6, 128.7 ,129.0, 129.4, 129.5, 137.5, 138.8, 142.4, 150.0, 162.7; Anal. Calcd for $\mathrm{C}_{37} \mathrm{H}_{42} \mathrm{OSiSn}$ : C, 68.42; H, 6.52, found: $\mathrm{C}, 68.95 ; \mathrm{H}, 6.70$. As shown in Figure 1, nOe spectra confirmed the double bond geometry depicted.

Table 1, Entry 6. To a solution of $\mathrm{Pd}(\mathrm{PhCN})_{2} \mathrm{Cl}_{2}(1.9 \mathrm{mg}, 0.0050 \mathrm{mmol} \mathrm{Pd}), \mathrm{P}\left(\mathrm{C}_{6} \mathrm{~F}_{5}\right)_{3}(5.3 \mathrm{mg}$, $0.0100 \mathrm{mmol}$ ), and $\mathrm{Bu}_{3} \mathrm{Sn}_{-} \mathrm{SiMe}_{3}(40.0 \mathrm{mg}, 0.110 \mathrm{mmol})$ in $1 \mathrm{~mL}$ of $\mathrm{C}_{6} \mathrm{D}_{6}$ at $\mathrm{rt}$ was added allenyne 3 (18.4 mg, $0.100 \mathrm{mmol})$ and the reaction mixture was kept in an NMR tube at rt. Progress of the reaction was followed by ${ }^{1} \mathrm{H}$ NMR. After $14 \mathrm{~h}$ at $\mathrm{rt},{ }^{1} \mathrm{H}$ NMR of the mixture showed a mixture of 3 and product 28 (ratio 3:10) and no further progress until $22 \mathrm{~h}$ at $\mathrm{rt}$. Then the reaction mixture was heated at $80{ }^{\circ} \mathrm{C}$ for $5 \mathrm{~h}$, and ${ }^{1} \mathrm{H}$ NMR of the mixture showed a clean conversion into cyclic product. Solvent was evaporated from the contents of the NMR tube and the residual oil was purified by flash chromatography $\left(\mathrm{Hex} / \mathrm{Et}_{3} \mathrm{~N}=95 / 5\right)$ followed by a careful second chromatography to get 40 mg (72\%) of product as a colorless oil. 28. IR (thin film): 2956, 2924, 1622, 1518, 1484, 1417, 1376, 1292, 1248, 1092, 1050, 982, $933 \mathrm{~cm}^{-1} ;{ }^{1} \mathrm{H}$ NMR (500 MHz, $\left.\mathrm{CDCl}_{3}\right)$ : $\square 0.20$ (s, 9H), 0.75$0.92\left(\mathrm{~m}, \mathrm{Sn} B u_{3}\right), 1.20-1.50$ (m, SnBu $), 3.48$ (s br, H, CH(TMS)C=CH ), 4.47 (dd, $J=1.6,12.6 \mathrm{~Hz}$, $\left.\mathrm{H}, \mathrm{CH}_{2} \mathrm{C}=\mathrm{C}\right), 4.56\left(\mathrm{td}, J=1.6,12.6 \mathrm{~Hz}, \mathrm{H}, \mathrm{CH}_{2} \mathrm{C}=\mathrm{C}\right), 4.98(\mathrm{~d}, J=2.1 \mathrm{~Hz}, \mathrm{H}, \mathrm{OCH}), 5.57(\mathrm{dd}, J=$ 
$\left.0.9,2.3 \mathrm{~Hz}, \mathrm{SiC}=\mathrm{CH}_{2}\right), 5.75\left(\mathrm{t}, J=1.8 \mathrm{~Hz}, \mathrm{H}, \mathrm{SiC}=\mathrm{CH}_{2}\right), 5.96\left(\mathrm{~d}, J=1.5 \mathrm{~Hz},{ }^{2} J_{\mathrm{Sn}-\mathrm{H}}=56 \mathrm{~Hz}, \mathrm{H}\right.$, $\mathrm{CHSn}) ;{ }^{13} \mathrm{C}$ NMR (125 MHz, $\mathrm{CDCl}_{3}$ ): $\square 0.4,10.4,14.1,27.8,29.5,59.8,74.3,86.8,120.8,126.4$, 127.6, 127.8, 128.8, 142.8, 152.6, 159.3; HRMS (FAB) calcd for $\mathrm{C}_{28} \mathrm{H}_{48} \mathrm{OSnSi}\left[(\mathrm{M}+\mathrm{Na})^{+}\right]$ 571.2394, found 571.2395. For nOe data see Figure 1.

Reactions of Cyclization Products with Elctrophilic Reagents $\left(\mathrm{Br}_{2}\right.$ and $\left.\mathrm{H}^{+}\right)$. (a) Reaction with Bromine. To a solution of Sn-Si adduct $27(25.8 \mathrm{mg}, 0.03972 \mathrm{mmol})$ in $\mathrm{CH}_{2} \mathrm{Cl}_{2}(1 \mathrm{~mL})$ was added $\mathrm{N}$-bromosuccinimide (10.6 $\mathrm{mg},(0.05958 \mathrm{mmol}, 1.5$ equiv.) at rt. The reaction mixture was stirred for $1 \mathrm{~h}$ and concentrated, then was subject to column chromatography $(\mathrm{EtOAc} / \mathrm{Hex}=1 / 9)$ to get 11 mg of an oil (74 \%). 27-Br: ${ }^{1} \mathrm{H}$ NMR (500 MHz, $\mathrm{CDCl}_{3}$ ): $\square 0.10$ (s, 3H), 0.14 (s, 3H), 0.94 (s, 9H), $3.90(\mathrm{~s} \mathrm{br}, \mathrm{H}), 4.48$ (dd, $J=2.0,3.6 \mathrm{~Hz}, 2 \mathrm{H}), 4.90(\mathrm{~d}, J=2.7 \mathrm{~Hz}, \mathrm{H}), 5.60(\mathrm{~d}, J=1.7 \mathrm{~Hz}, \mathrm{H}), 5.78$ (t, $J=1.4 \mathrm{~Hz}, \mathrm{H}), 6.20$ (q, $J=1.9 \mathrm{~Hz}, \mathrm{H}), 7.27-7.50(\mathrm{M}, 5 \mathrm{H}) ;{ }^{13} \mathrm{C} \mathrm{NMR}\left(125 \mathrm{MHz}, \mathrm{CDCl}_{3}\right)$ : $\square-4.1$, $-4.4,17.7,27.4,57.4,71.6,87.5,98.5$, aromatic carbons are omitted, HRMS calcd for [M+Na] 401.0912, found: 401.0903.

(b) Reaction of 27 with Formic Acid. A crude reaction mixture of a solution of [Sn-Si]-adduct 27 (64.9 mg, $0.10 \mathrm{mmol}$, theory) in $\mathrm{CH}_{2} \mathrm{Cl}_{2}(1 \mathrm{~mL})$ was treated with $\mathrm{HCO}_{2} \mathrm{H}(23 \mathrm{mg}, 0.50 \mathrm{mmol})$ overnight. The solvent was evaporated and the residue was purified by flash column chromatography (EtOAc/Hex $=1 / 2)$ to get $23 \mathrm{mg}(78 \%)$ of an oil. $27-\mathrm{H} .{ }^{1} \mathrm{H}$ NMR $(400 \mathrm{MHz}$, $\mathrm{CDCl}_{3}$ ): $\square-0.42$ (s, 3H), -0.01 (s. $3 \mathrm{H}$ ), 0.78 (s, 9H), 3.32 (d br, $J=8.9 \mathrm{~Hz}, \mathrm{H}$ ), 4.57 (ddd, $J=2.1$, 4.3, 13.2 Hz, H), 4.65 (d, $J=8.9 \mathrm{~Hz}, \mathrm{H}), 4.76(\mathrm{ddd}, J=2.2,3.3,13.2 \mathrm{~Hz}, \mathrm{H}), 4.87$ (dd, $J=2.4,4.9$ $\mathrm{Hz}, \mathrm{H}), 5.00(\mathrm{dd}, J=2.1,4.8 \mathrm{~Hz}, \mathrm{H}), 5.75(\mathrm{~d}, J=2.2 \mathrm{~Hz}, \mathrm{H}), 5.97(\mathrm{~d}, J=2.1 \mathrm{~Hz}, \mathrm{H}) ;{ }^{13} \mathrm{C}$ NMR (100 MHz, $\left.\mathrm{CDCl}_{3}\right)$ : $\square-6.8,-5.8,17.0,26.5,57.9,71.7,88.4,105.3,125.7,127.7,128.1,128.4$, 130.9, 136.9, 137.1; HRMS calcd for [M+Na] 323.1807, found 323.1812.

\section{Cyclization of Substrate 5 (Table 1, Entry 8). trans-Z-[3,4]-Bicyclo-1-N-2-oxo-7-[(tri-n-} butylstannyl)methylene]-6-[(1-trimethylsilyl)ethene]-octane (30). To a solution of 5-allenyl-1(3-butynyl)-2-pyrrolidinone (15 mg, $0.08 \mathrm{mmol}$ ) in $1 \mathrm{~mL} \mathrm{C}_{6} \mathrm{D}_{6}$ were added $\mathrm{Bu}_{3} \mathrm{SnSiMe}_{3}(29 \square \mathrm{L}$, $0.08 \mathrm{mmol}, 1$ equiv.), $\mathrm{Pd}_{2}(\mathrm{dba})_{3}(1.5 \mathrm{mg}, 0.002 \mathrm{mmol}, 2 \mathrm{~mol} \%)$ and $\left.\mathrm{P}_{\left(\mathrm{C}_{6}\right.} \mathrm{F}_{5}\right)_{3}(5.0 \mathrm{mg}, 0.008 \mathrm{mmol}$, $10 \mathrm{~mol} \%$ ). The reaction was monitored by ${ }^{1} \mathrm{H} N M R$. After $18 \mathrm{~h}$ at $60^{\circ} \mathrm{C}$, the reaction was complete (100\% yield by NMR). The solvent was evaporated and the crude mixture purified by column chromatography $\left(\mathrm{SiO}_{2}\right.$ : hexanes / diethyl ether 1/1) to give $5 \mathrm{mg}$ (11\% isolated yield) of a colorless oil. The same product could be obtained in $41 \%$ yield by reaction of 5-(1-E-3-tri- $n$-butylstannyl-2trimethylsilylpropenyl)-1-(3-butynyl)-2-pyrrolidinone with $2 \mathrm{~mol} \%$ of $\mathrm{Pd}_{2}\left(\mathrm{dba}_{3}\right.$ and $10 \mathrm{~mol} \%$ of 
$\mathrm{P}\left(\mathrm{C}_{6} \mathrm{~F}_{5}\right)_{3}$ in $1 \mathrm{~mL}$ of $\mathrm{C}_{6} \mathrm{D}_{6} .{ }^{1} \mathrm{H} \mathrm{NMR}\left(500 \mathrm{MHz}, \mathrm{CDCl}_{3}\right)$ : $\square 5.81\left(\mathrm{~s}, \mathrm{~J}_{\mathrm{Sn}-\mathrm{H}}=28.0,1 \mathrm{H}, \mathrm{SnCH}\right), 5.67$ $\left(\mathrm{dm}, \mathrm{J}=2.5,1 \mathrm{H}, \mathrm{C}=\mathrm{CH}_{2}\right), 5.50\left(\mathrm{~d}, \mathrm{~J}=2.6,1 \mathrm{H}, \mathrm{C}=\mathrm{CH}_{2}\right), 3.82\left(\mathrm{ddd}, \mathrm{J}=8.6,6.7,1.3,1 \mathrm{H}, H^{5}\right), 3.64$ $\left(\mathrm{m}, 1 \mathrm{H}, H^{8}\right), 3.23\left(\mathrm{~m}, 1 \mathrm{H}, H^{9}\right), 2.98\left(\mathrm{~d}, \mathrm{~J}=8.6,1 \mathrm{H}, H^{9}\right), 2.71\left(\mathrm{~m}, 1 \mathrm{H}, H^{6}\right), 2.41\left(\mathrm{~m}, 1 \mathrm{H}, H^{8}\right), 2.34$ $\left(\mathrm{t}, \mathrm{J}=7.5,2 \mathrm{H}, H^{3}\right), 2.14\left(\mathrm{~m}, 1 \mathrm{H}, H^{4}\right), 1.62\left(\mathrm{~m}, 1 \mathrm{H}, H^{4}\right), 1.42\left(\mathrm{~m}, 6 \mathrm{H}, \mathrm{SnCH}_{2}\right), 1.27(\mathrm{~m}, 6 \mathrm{H}$, $\left.\mathrm{SnCH}_{2} \mathrm{CH}_{2}\right), 0.85\left(\mathrm{~m}, 15 \mathrm{H}, \mathrm{Sn}\left(\mathrm{CH}_{2}\right)_{2} \mathrm{CH}_{2} \mathrm{CH}_{3}\right), 0.13$ (s, $\left.9 \mathrm{H}, \mathrm{SiCH}_{3}\right) ;$ nOe : $\mathrm{SnCH} \square H^{8}: 3 \%$, $\mathrm{SnCH} \square H^{6}: 0 \%, H^{5} \square H^{6}: 0 \% ;{ }^{13} \mathrm{C} \mathrm{NMR}\left(125 \mathrm{MHz}, \mathrm{CDCl}_{3}\right): \square 174.2,153.9,150.5,129.1$, $128.9,60.3,58.6,41.0,36.1,30.5,29.1,27.4,25.3,13.7,10.6,0.4 ;{ }^{119} \mathrm{Sn}\left(186 \mathrm{MHz}, \mathrm{CDCl}_{3}\right) \square-$ 66.3; IR (film): 1671, 1520, $1422 \mathrm{~cm}^{-1}$; HRMS calcd for $\mathrm{C}_{26} \mathrm{H}_{49} \mathrm{NOSiSnNa:} \mathrm{[M]} \mathrm{=} \mathrm{562.24977,}$ found 562.25382. See Figure 1 for nOe data which supports the stereochemical assignments. Cyclization of Substrate 6 (Table 1, Entry 9). trans-Z-[3,4]-Bicyclo-1-N-2-oxo-8-[(1-dimethylt-silyl)ethene]-7-[(triphenylstannyl)methylene]-octane (31). To a solution of 1-(2,3-butadienyl)5-(2-propynyl)-2-pyrrolidinone (20 mg, $0.11 \mathrm{mmol})$ in $1 \mathrm{~mL} \mathrm{C}_{6} \mathrm{D}_{6}$ were added $\mathrm{Ph}_{3} \mathrm{SnSiMe}_{2}-t$-Bu (52 mg, $0.11 \mathrm{mmol}, 1$ equiv.), $\mathrm{Pd}_{2}(\mathrm{dba})_{3}(2 \mathrm{mg}, 0.002 \mathrm{mmol}, 2 \mathrm{~mol} \%)$ and $\mathrm{P}\left(\mathrm{C}_{6} \mathrm{~F}_{5}\right)_{3}(6 \mathrm{mg}, 0.011 \mathrm{mmol}$, $10 \mathrm{~mol} \%$ ). The reaction was monitored by ${ }^{1} \mathrm{H} \mathrm{NMR}$. After $18 \mathrm{~h}$ at $40^{\circ} \mathrm{C}$, the reaction was complete (100\% yield by NMR). The solvent was evaporated and the crude mixture purified by column chromatography $\left(\mathrm{SiO}_{2}\right.$ : hexanes / diethyl ether 2/1) to give $29 \mathrm{mg}$ (41\% isolated yield) of $\mathbf{3 1}$ as a colorless oil. The same product could be obtained by reaction of 5-(2-propynyl)-1-(2-E-4-tri- $n$ butylstannyl-3-trimethylsilylbutenyl)-2-pyrrolidinone (the corresponding acyclic adduct) with 2 mol\% of $\mathrm{Pd}_{2}(\mathrm{dba})_{3}$ and $10 \mathrm{~mol} \%$ of $\mathrm{P}\left(\mathrm{C}_{6} \mathrm{~F}_{5}\right)_{3}$ in $1 \mathrm{~mL}$ of $\mathrm{C}_{6} \mathrm{D}_{6} .{ }^{1} \mathrm{H} \mathrm{NMR}\left(500 \mathrm{MHz}, \mathrm{C}_{6} \mathrm{D}_{6}\right): \square 7.60$ $(\mathrm{dd}, \mathrm{J}=7.8,1.4,6 \mathrm{H}, \mathrm{Sn} P h(o)), 7.20(\mathrm{~m}, 9 \mathrm{H}, \mathrm{Sn} P h(m+p)), 6.04\left(\mathrm{~s}, \mathrm{~J}_{\mathrm{Sn}-\mathrm{H}}=28.0,1 \mathrm{H}, \mathrm{SnCH}\right), 5.84$ $\left(\mathrm{t}, \mathrm{J}=2.1,1 \mathrm{H}, \mathrm{SiCCH}_{2}\right), 5.45\left(\mathrm{t}, \mathrm{J}=2.1,1 \mathrm{H}, \mathrm{SiCCH}_{2}\right), 3.92\left(\mathrm{dd}, \mathrm{J}=13.2,6.7,1 \mathrm{H}, H^{9}\right), 3.57(\mathrm{~m}, 1$ $\left.\mathrm{H}, H^{8}\right), 3.50\left(\mathrm{~m}, 1 \mathrm{H}, H^{5}\right), 3.23\left(\mathrm{dd}, \mathrm{J}=13.2,5.2,1 \mathrm{H}, H^{9}\right), 2.52\left(\mathrm{dd}, \mathrm{J}=6.0,1.6,1 \mathrm{H}, H^{4}\right), 2.06-1.96$ (m, $2 \mathrm{H}, H^{6}$ overlapping m, $\left.1 \mathrm{H}, H^{4}\right), 1.34\left(\mathrm{~m}, 1 \mathrm{H}, H^{3}\right), 1.09\left(\mathrm{~m}, 1 \mathrm{H}, H^{3}\right), 0.75(\mathrm{~s}, 9 \mathrm{H}, \mathrm{Si} B u),-0.17$ $\left(\mathrm{s}, 3 \mathrm{H}, \mathrm{SiCH}_{3}\right),-0.24$ (s, $\left.3 \mathrm{H}, \mathrm{SiCH}_{3}\right)$; nOe : $\mathrm{SnCH} \square H^{6}: 3 \%, \mathrm{SnCH} \square H^{8}: 0 \%, H^{5} \square \mathrm{Si} B u$ : 1\%, $H^{5} \square H^{8}: 0 \%$; See Figure 1 for nOe data. $\left.{ }^{13} \mathrm{C} \mathrm{NMR} \mathrm{(125} \mathrm{MHz,} \mathrm{CDCl}_{3}\right): \square 173.4,156.8$, 151.9, 139.1, 137.4, 129.3, 129.0, 125.5, 53.6, 50.7, 44.0, 42.0, 31.1, 27.3, 26.8, 17.3, -3.4, -5.5; ${ }^{119} \mathrm{Sn}\left(186 \mathrm{MHz}, \mathrm{C}_{6} \mathrm{D}_{6}\right) \square-152.0$; IR (film): 1682, $1428 \mathrm{~cm}^{-1}$; HRMS calcd for $\mathrm{C}_{35} \mathrm{H}_{43} \mathrm{NOSiSnNa}$ : $[\mathrm{M}]=664.202829$, found 664.207532.

\section{Reaction of allenyne 5 with $\mathrm{Me}_{3} \mathrm{SiSnBu}_{3}$ (Table 4, Entry 5, Eq 10). 5-(1-E-3-Tri-n-} butylstannyl-2-trimethylsilylpropenyl)-1-(3-butynyl)-2-pyrrolidinone (38). To a solution of 5allenyl-1-(3-butynyl)-2-pyrrolidinone (15 mg, $0.08 \mathrm{mmol}$ ) in $1 \mathrm{~mL} \mathrm{C}_{6} \mathrm{D}_{6}$ were added $\mathrm{Bu}_{3} \mathrm{SnSiMe}_{3}$ 
(29 $\square \mathrm{L}, 0.08 \mathrm{mmol}, 1$ equiv.), $\mathrm{Pd}_{2}(\mathrm{dba})_{3}(1.5 \mathrm{mg}, 0.002 \mathrm{mmol}, 2 \mathrm{~mol} \%)$ and $\mathrm{P}\left(\mathrm{C}_{6} \mathrm{~F}_{5}\right)_{3}(5.0 \mathrm{mg}, 0.008$ mmol, $10 \mathrm{~mol} \%$ ). The reaction was monitored by ${ }^{1} \mathrm{H}$ NMR. After 15 minutes at room temperature, the reaction was complete ( $100 \%$ yield by NMR). The solvent was evaporated and the crude mixture purified by column chromatography $\left(\mathrm{SiO}_{2}\right.$ : hexanes / diethyl ether 1/1) to give $20 \mathrm{mg}(44 \%$ isolated yield) of a colorless oil. ${ }^{1} \mathrm{H} \mathrm{NMR}\left(500 \mathrm{MHz}, \mathrm{CDCl}_{3}\right)$ : $\square 5.20\left(\mathrm{q}, \mathrm{J}=9.1,1 \mathrm{H}, \mathrm{CH}^{5} \mathrm{CH}\right), 4.43$ $\left(\mathrm{m}, 1 \mathrm{H}, H^{5}\right.$ ), 3.52 (quintuplet, $\mathrm{J}=6.9,1 \mathrm{H}, \mathrm{NCH}_{2}$ ), 3.05 (quintuplet, $\mathrm{J}=7.2,1 \mathrm{H}, \mathrm{NCH}_{2}$ ), $2.46(\mathrm{~m}, 1$ $\left.\mathrm{H}, H^{4}\right), 2.35\left(\mathrm{~m}, 3 \mathrm{H}, H^{4}+\mathrm{NCH}_{2} \mathrm{CH}_{2}\right), 2.18\left(\mathrm{~m}, 1 \mathrm{H}, H^{3}\right), 2.11\left(\mathrm{dd}, \mathrm{J}=7.9,2.1,1 \mathrm{H}, H^{3}\right), 1.91(\mathrm{t}, \mathrm{J}=$ 2.7, $1 \mathrm{H}, \mathrm{CCH}), 1.74$ (d, J = 11.3, $\left.1 \mathrm{H}, \mathrm{SnCH}_{2} \mathrm{CSi}\right), 1.63$ (m, $\left.1 \mathrm{H}, \mathrm{SnCH}_{2} \mathrm{CSi}\right), 1.45$ (m, $6 \mathrm{H}$, $\left.\mathrm{SnCH}_{2}\right), 1.28\left(\mathrm{~m}, 6 \mathrm{H}, \mathrm{SnCH}_{2} \mathrm{CH}_{2}\right), 0.88\left(\mathrm{~m}, 15 \mathrm{H}, \mathrm{Sn}\left(\mathrm{CH}_{2}\right)_{2} \mathrm{CH}_{2} \mathrm{CH}_{3}\right), 0.05$ (s, $\left.9 \mathrm{H}, \mathrm{SiCH}_{3}\right) ;$ nOe : $\mathrm{CH}^{5} \mathrm{CH} \square \mathrm{SiMe}$ : 2\%; ${ }^{13} \mathrm{C} \mathrm{NMR}\left(125 \mathrm{MHz}, \mathrm{CDCl}_{3}\right): \square 175.0,146.6,131.8,81.6,69.6,56.2,39.8$, 30.3, 29.1, 27.4, 25.7, 17.9, 13.6, 12.9, 10.1, -1.6; ${ }^{119} \mathrm{Sn}\left(186 \mathrm{MHz}, \mathrm{CDCl}_{3}\right) \square-18.6$; IR (film): $3312,1681,1455,1417 \mathrm{~cm}^{-1}$. This compound was fully characterized as the bicyclic adduct 30.

Table 3, Entry 1. To a solution of $\mathrm{Pd}_{2}(\mathrm{dba})_{3} \mathrm{CHCl}_{3}(2.6 \mathrm{mg}, 0.0050 \mathrm{mmol} \mathrm{Pd}), \mathrm{P}\left(\mathrm{C}_{6} \mathrm{~F}_{5}\right)_{3}(5.3 \mathrm{mg}$, $0.010 \mathrm{mmol})$, and $\mathrm{Me}_{3} \mathrm{Sn}-\mathrm{B}\left(-\mathrm{N}(\mathrm{Me}) \mathrm{CH}_{2} \mathrm{CH}_{2}(\mathrm{Me}) \mathrm{N}-\right)(26.0 \mathrm{mg}, 0.100 \mathrm{mmol})$ in $1 \mathrm{~mL}$ of $\mathrm{C}_{6} \mathrm{D}_{6}$ at rt was added allenyne $1 \mathbf{a}(25.0 \mathrm{mg}, 0.100 \mathrm{mmol})$ and the reaction mixture was kept in an NMR tube. Progress of the reaction was followed by ${ }^{1} \mathrm{H}$ NMR. After $17 \mathrm{~h}$ at $\mathrm{rt}$, the ${ }^{1} \mathrm{H}$ NMR spectrum showed reasonable conversion into the product $\mathbf{4 2}$ along with some side-product with less than $10 \%$ of starting 1a remaining. However all attempts to isolate this product failed. The following data was extracted from the in situ ${ }^{1} \mathrm{H}$ NMR spectra taken in $\mathrm{C}_{6} \mathrm{D}_{6}$. 42. ${ }^{1} \mathrm{H}$ NMR (500 MHz, $\left.\mathrm{C}_{6} \mathrm{D}_{6}\right)$ : $\square 5.69$ (d, $J=2.4 \mathrm{~Hz}, J_{\mathrm{Sn}-\mathrm{H}}=$ not determined, $\left.\mathrm{H}, \mathrm{RC}(\mathrm{Sn})=\mathrm{CH}_{2}\right), 5.60(\mathrm{~s}, \mathrm{H}, \mathrm{C}=\mathrm{CHB}), 5.15\left(\mathrm{~d}, J=2.5 \mathrm{~Hz}, J_{\mathrm{Sn}-\mathrm{H}}\right.$ $\left.=64 \mathrm{~Hz}, \mathrm{H}, \mathrm{RC}(\mathrm{Sn})=\mathrm{CH}_{2}\right)$.

Addition of Distannane to Allenyne 1a (Table 3, Entries 2/3). To a solution of $\mathrm{Pd}_{2}(\mathrm{dba})_{3} \mathrm{CHCl}_{3}$ (1.3 mg, $0.0025 \mathrm{mmol} \mathrm{Pd}), \mathrm{P}\left(\mathrm{C}_{6} \mathrm{~F}_{5}\right)_{3}(2.7 \mathrm{mg}, 0.0050 \mathrm{mmol})$, and $\mathrm{Me}_{3} \mathrm{Sn}-\mathrm{SnMe}_{3}(18.0 \mathrm{mg}, 0.055$ $\mathrm{mmol})$ in $1 \mathrm{~mL}$ of $\mathrm{C}_{6} \mathrm{D}_{6}$ at $\mathrm{rt}$ was added allenyne $1 \mathrm{a}(12.5 \mathrm{mg}, 0.050 \mathrm{mmol})$ and the reaction mixture was kept in an NMR tube. Progress of the reaction was followed by ${ }^{1} \mathrm{H} \mathrm{NMR}$. After $22 \mathrm{~h}$ at $\mathrm{rt}$, the reaction mixture showed a clean conversion into the acyclic product 44 , which after 2 days at $45^{\circ} \mathrm{C}$, was converted into 43 (>90\%) with $<10 \%$ of starting 44 remaining. Solvent was evaporated from the contents of the NMR tube and the residual oil was purified by flash chromatography $\left(\mathrm{Hex} / \mathrm{Et}_{3} \mathrm{~N}\right.$ $=97 / 3)$ to get $13 \mathrm{mg}(46 \%)$ of product as a colorless oil. 44. ${ }^{1} \mathrm{H} \mathrm{NMR}\left(500 \mathrm{MHz}, \mathrm{CDCl}_{3}\right): \square 0.08$ $(\mathrm{s}, 9 \mathrm{H}), 0.11(\mathrm{~s}, 9 \mathrm{H}), 0.87(\mathrm{~s}, J=7.0 \mathrm{~Hz}, 3 \mathrm{H}), 1.67\left(\mathrm{t}, J=2.7 \mathrm{~Hz}, \mathrm{H}, \mathrm{CH}_{2} \mathrm{C} \_\mathrm{CH}\right), 2.23\left(\mathrm{~s}, J_{\mathrm{Sn}-\mathrm{H}}=72\right.$ 
$\left.\mathrm{Hz},\left(\mathrm{Me}_{3} \mathrm{Sn}\right) \mathrm{CH}_{2} \mathrm{C}\left(\mathrm{SnMe}_{3}\right)=\mathrm{CH}, 2 \mathrm{H}\right), 3.08\left(\mathrm{~d}, J=2.7 \mathrm{~Hz}, 2 \mathrm{H}, \mathrm{CH}_{2} \mathrm{C} \_\mathrm{CH}\right), 3.18(\mathrm{~d}, J=6.7 \mathrm{~Hz}, 2 \mathrm{H}$, $\left.\mathrm{C}\left(\mathrm{SnMe}_{3}\right)=\mathrm{CHCH}_{2}\right), 3.91(\mathrm{~m}, 4 \mathrm{H}), 5.34\left(\mathrm{t}, J=6.7 \mathrm{~Hz}, \mathrm{H}, \mathrm{C}\left(\mathrm{SnMe}_{3}\right)=\mathrm{CHCH}_{2}\right) .43 .{ }^{1} \mathrm{H} \mathrm{NMR}(500$ $\left.\mathrm{MHz}, \mathrm{CDCl}_{3}\right): \square 0.06\left((\mathrm{~s}, 9 \mathrm{H}), 0.17(\mathrm{~s}, 9 \mathrm{H}), 1.24\left(\mathrm{t}, J=7.0 \mathrm{~Hz}, 6 \mathrm{H}, \mathrm{OCH}_{2} \mathrm{CH}_{3}\right), 2.00\right.$ (dd, $J=7.8$, $13.2 \mathrm{~Hz}, \mathrm{H}$, ring $\mathrm{CH}_{2} \mathrm{CH}$ ), 2.72 (ddd, $J=1.6,8.6,13.2 \mathrm{~Hz}, \mathrm{H}$, ring $\mathrm{CH}_{2} \mathrm{CH}$ ), 2.90 (dd, $J=1.5,17.5$ $\mathrm{Hz}, \mathrm{H}$, ring $\mathrm{CH}_{2}$ ), 3.15 (td, $J=2.4,16.0 \mathrm{~Hz}, \mathrm{H}$, ring $\mathrm{CH}_{2}$ ), 3.45 (t br, $J=7.9 \mathrm{~Hz}, \mathrm{H}, \mathrm{CH}_{2} \mathrm{CH}$ ), 4.10$4.23\left(\mathrm{~m}, 4 \mathrm{H}, \mathrm{OCH}_{2} \mathrm{CH}_{3}\right), 5.20\left(\mathrm{dd}, J=2.2,0.9 \mathrm{~Hz},{ }^{3} J_{\mathrm{Sn}-\mathrm{H}}=68 \mathrm{~Hz}, \mathrm{H}, \mathrm{SnC}=\mathrm{CH}_{2}\right), 5.70(\mathrm{dd}, J=2.2$, $\left.1.3 \mathrm{~Hz},{ }^{3} J_{\mathrm{Sn}-\mathrm{H}}=150 \mathrm{~Hz}, \mathrm{SnC}=\mathrm{CH}_{2}\right), 5.91\left(\mathrm{~s},{ }^{2} J_{\mathrm{Sn}-\mathrm{H}}=66 \mathrm{~Hz}, \mathrm{CHSn}\right) ;{ }^{13} \mathrm{C} \mathrm{NMR}\left(125 \mathrm{MHz}, \mathrm{CDCl}_{3}\right)$ : $\square-$ 7.8, -7.7, 14.5, 14.4, 41.7, 46.2, 53.9, 59.6, 61.8, 61.9, 124.8, 126.0, 157.1, 159.2, 171.2, 171.9; ${ }^{119} \mathrm{Sn}$ NMR (186 MHz, $\mathrm{CDCl}_{3}$ ): $\square-31.1,-55.6$; HRMS (FAB) calcd for $\mathrm{C}_{20} \mathrm{H}_{36} \mathrm{O}_{4} \mathrm{Sn}_{2} \mathrm{Na}[(\mathrm{M}+\mathrm{Na})]$ 601.0558 , found 601.0548 .

Addition of Hexamethylditin to 3 (Table 3, Entry 4). To a solution of $\mathrm{Pd}(\mathrm{PhCN})_{2} \mathrm{Cl}_{2}(1.9 \mathrm{mg}$, $0.0050 \mathrm{mmol} \mathrm{Pd}), \mathrm{P}\left(\mathrm{C}_{6} \mathrm{~F}_{5}\right)_{3}(5.3 \mathrm{mg}, 0.010 \mathrm{mmol})$, and $\mathrm{Me}_{3} \mathrm{Sn}-\mathrm{SnMe}_{3}(36.3 \mathrm{mg}, 0.110 \mathrm{mmol})$ in 1 $\mathrm{mL}$ of $\mathrm{C}_{6} \mathrm{D}_{6}$ at $\mathrm{rt}$ was added allenyne $3(18.4 \mathrm{mg}, 0.100 \mathrm{mmol})$ and the reaction mixture was kept in

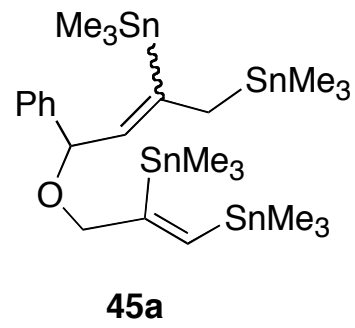
an NMR tube at rt. Progress of the reaction was followed by ${ }^{1} \mathrm{H}$ NMR. After $14 \mathrm{~h}$ at $\mathrm{rt},{ }^{1} \mathrm{H}$ NMR of the reaction mixture indicated a mixture of acyclic bisadduct $\mathbf{4 5 a}$ and cyclic product $\mathbf{4 5}$. Under more forcing conditions, the reaction did not progress further $\left(80^{\circ} \mathrm{C}, 5 \mathrm{~h}\right)$. Solvent was evaporated from the contents of the NMR tube and the residual oil was purified by flash chromatography $\left(\mathrm{Hex} / \mathrm{Et}_{3} \mathrm{~N}=97 / 3\right)$ to get $29 \mathrm{mg}(42 \%)$ of mixture of products as a colorless oil. Investigation of ${ }^{1} \mathrm{H}$ NMR spectrum of this sample indicated it to be a mixture of $\mathbf{4 5}$ and $\mathbf{4 5 a}$ (molar ratio of 1:1). The following ${ }^{1} \mathrm{H}$ NMR spectra for $\mathbf{4 5}$ and $\mathbf{4 5 a}$ were extracted from that of sample of the above mixture. Owing to the presence of multiple $\mathrm{SnMe}_{3}$ moiety, the $J_{\mathrm{Sn}-\mathrm{H}}$ coupling was not very clear. Because of incomplete characterization, the structure of the products must be considered tentative. 45a. ${ }^{1} \mathrm{H} \mathrm{NMR}\left(500 \mathrm{MHz}, \mathrm{CDCl}_{3}\right): \square 0.10$ (s, 9H), 0.11 (s, 9H), 0.16 (s, 9H), 0.18 (s, 9H), 2.11 (dd, $\left.J=0.9,11.2 \mathrm{~Hz}, \mathrm{H},(\mathrm{Sn}) \mathrm{CH}_{2} \mathrm{C}(\mathrm{Sn})=\mathrm{CHCH}(\mathrm{Ph}) \mathrm{O}\right), 2.32$ $\left(\mathrm{dd}, J=1.0,11.2 \mathrm{~Hz}, \mathrm{H},(\mathrm{Sn}) \mathrm{CH}_{2} \mathrm{C}(\mathrm{Sn})=\mathrm{CHCH}(\mathrm{Ph}) \mathrm{O}\right), 4.50\left(\mathrm{t}, J=2.0 \mathrm{~Hz}, \mathrm{H}, \mathrm{OCH}_{2} \mathrm{C}(\mathrm{Sn})\right.$ $=\mathrm{CH}(\mathrm{Sn})), 4.53\left(\mathrm{~d}, J=1.6 \mathrm{~Hz}, \mathrm{H}, \mathrm{OCH}_{2} \mathrm{C}(\mathrm{Sn})=\mathrm{CH}(\mathrm{Sn})\right), 5.02(\mathrm{~d}, J=7.8 \mathrm{~Hz}, \mathrm{H}$, $\left.(\mathrm{Sn}) \mathrm{CH}_{2} \mathrm{C}(\mathrm{Sn})=\mathrm{CHCH}(\mathrm{Ph}) \mathrm{O}\right), 5.52\left(\mathrm{~d}, J=7.8 \mathrm{~Hz}, \mathrm{H},(\mathrm{Sn}) \mathrm{CH}_{2} \mathrm{C}(\mathrm{Sn})=\mathrm{CHCH}(\mathrm{Ph}) \mathrm{O}\right), 6.86\left(\mathrm{~s}, J_{\mathrm{Sn}-\mathrm{H}}=\right.$ $\left.60 \mathrm{~Hz}, \mathrm{H}, \mathrm{OCH}_{2} \mathrm{C}(\mathrm{Sn})=\mathrm{CH}(\mathrm{Sn})\right)$. 45. ${ }^{1} \mathrm{H} \mathrm{NMR}\left(500 \mathrm{MHz} \mathrm{CDCl}_{3}\right): \square 0.02(\mathrm{~s}, 9 \mathrm{H}), 0.21(\mathrm{~s}, 9 \mathrm{H})$, $3.53\left(\mathrm{~d}, J=5.7 \mathrm{~Hz}, \mathrm{H}, \mathrm{CHC}(\mathrm{Sn})=\mathrm{CH}_{2}\right), 3.97\left(\mathrm{dd}, J=1.5,11.5 \mathrm{~Hz}, \mathrm{H}, \mathrm{CH}_{2} \mathrm{C}=\mathrm{C}(\mathrm{Sn}) \mathrm{H}\right), 4.14(\mathrm{dd}, J$ 
$\left.=1.5,11.5 \mathrm{~Hz}, \mathrm{CH}_{2} \mathrm{C}=\mathrm{C}(\mathrm{Sn}) \mathrm{H}\right), 4.79(\mathrm{~d}, J=5.7 \mathrm{~Hz}, \mathrm{PhCHO}), 5.35(\mathrm{~d}, J=2.3 \mathrm{~Hz}, \mathrm{H}, \mathrm{CHC}(\mathrm{Sn})=\mathrm{C}$ $\left.H_{2}\right), 5.69\left(\mathrm{dd}, J=0.8,2.3 \mathrm{~Hz}, \mathrm{H}, \mathrm{CHC}(\mathrm{Sn})=\mathrm{CH}_{2}\right), 5.87(\mathrm{dd}, J=1.9 \mathrm{~Hz}, \mathrm{H}, \mathrm{C}=\mathrm{C}(\mathrm{Sn}) H)$.

Reaction of Allenyne 7 with Di-tin Reagents under Different Conditions to Get 1-(Hept-1-en4-yl)-5-(prop-2-ynyl)pyrrolidin-2-one 47 (Table 3, Entry 5 and Eq 12)

Reaction of allenyne 7 (1 mmol, $217 \mathrm{mg})$ with $\mathrm{n}-\mathrm{Bu}_{3} \mathrm{Sn}-\mathrm{SnBu}_{3}-\mathrm{n}(1.2 \mathrm{mmol}, 694 \mathrm{mg})$ using $[(\text { allyl }) \mathrm{PdCl}]_{2}(0.05 \mathrm{mmol}, 18 \mathrm{mg})$ as the catalyst in refluxing THF over $24 \mathrm{~h}$ or with $\mathrm{Me}_{3} \mathrm{Sn}-\mathrm{SnMe}_{3}$ (1.2 mmol, $381 \mathrm{mg})$ employing $\mathrm{Pd}_{2}(\mathrm{dba})_{3} \cdot \mathrm{CHCl}_{3}(0.05 \mathrm{mmol}, 51 \mathrm{mg})$ and $\mathrm{P}\left(\mathrm{C}_{6} \mathrm{~F}_{5}\right)_{3}(53 \mathrm{mg}, 0.1$ $\mathrm{mmol}$ ) in benzene at $50^{\circ} \mathrm{C}$ for $48 \mathrm{~h}$, and subsequent treatment with CSA (4 mmol, $\left.928 \mathrm{mg}\right)$ in $10 \mathrm{~mL}$ of $\mathrm{CH}_{3} \mathrm{CN}$ and $0.5 \mathrm{~mL}$ of water for $4 \mathrm{~h}$, purification by flash column chromatography (15\% EtOAc in hexanes) afforded 47 (66 mg, 30\%) as an oil: ${ }^{1} \mathrm{H}$ NMR (500 MHz) $\square 5.8-5.66(\mathrm{~m}, 1 \mathrm{H})$, 5.16-5.01 $(\mathrm{m}, 2 \mathrm{H}), 3.87-3.65(\mathrm{~m}, 2 \mathrm{H}), 2.6-2.34(\mathrm{~m}, 4 \mathrm{H}), 2.3-2.12(\mathrm{~m}, 3 \mathrm{H}), 2.01(\mathrm{t}, \mathrm{J}=3 \mathrm{~Hz}, 1 \mathrm{H}), 2-1.94(\mathrm{~m}$, $1 \mathrm{H}), 1.76-1.59$ and 1.52-1.45 (m, 2H), 1.39-1.31 (m, 1H), 1.28-1.21 (m, 1H), $0.91(\mathrm{t}, \mathrm{J}=7.5 \mathrm{~Hz}$, $3 \mathrm{H}) ;{ }^{13} \mathrm{C}$ NMR (125 MHz) $\square 174.1$ and 175.9, 136 and 135.9, 117.5 and 117.4, 80.6, 71.3, 57.2 and 56.9, 53.9 and 53.6, 38,8 and 38.8, 36.4, 30.9 and 30.7, 25.7 and 25.5, 24.9 and 24.8, 20.5 and 20.5, 14.3 and 14.2; HRMS (EI/CI) calcd for $[\mathrm{M}]=\mathrm{C}_{14} \mathrm{H}_{21} \mathrm{NONa}^{+}$242.151532, found 242.149686.

\section{Reaction of Allenyne 7 with Tri-n-butyltinhydride. 1-(Hept-1-en-4-yl)-5-(prop-2-}

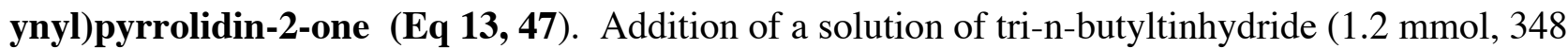
$\mathrm{mg})$ in toluene or benzene $(10 \mathrm{~mL})$ over $30 \mathrm{~min}$ to the mixture containing allenyne $(1 \mathrm{mmol}, 217$ $\mathrm{mg})$ and $\mathrm{Pd}(\mathrm{OAc})_{2}(0.05 \mathrm{mmol}, 11 \mathrm{mg})$ in toluene $(5 \mathrm{~mL})$ or $\mathrm{Pd}_{2}(\mathrm{dba})_{3} \cdot \mathrm{CHCl}_{3}(0.05 \mathrm{mmol}, 52 \mathrm{mg})$ in benzene $(5 \mathrm{~mL})$ at $\mathrm{rt}$, refluxing for $48 \mathrm{~h}$, concentration, protodestannylation as in previous cases employing CSA ( $4 \mathrm{mmol}, 928 \mathrm{mg}$ ) in $15 \mathrm{~mL}$ of $\mathrm{CH}_{3} \mathrm{CN}$ and $0.5 \mathrm{~mL}$ of water at rt over $4 \mathrm{~h}$ afforded $47(105 \mathrm{mg}, 48 \%)$ as an oil whose spectral data were in complete agreement with the data obtained for the product from the previous experiment.

Synthesis of 48 and Addition of Hexamethylditin to 48 (Table 3, Entry 6, Eq 14). (a) Synthesis of Allene 48 (Eq 14). To a solution of bis-THP ether $(2.54 \mathrm{~g}, 10.0 \mathrm{mmol})$ in ether $(20 \mathrm{~mL})$ was added LAH (455 mg, $12.0 \mathrm{mmol}$ ) and the resulting suspension was refluxed for $4 \mathrm{~h}$. The reaction mixture was quenched with $\sim 2 \mathrm{~g}$ of celite/ $\mathrm{Na}_{2} \mathrm{SO}_{4} /$ water $(3 / 3 / 1)$ mixture and was further stirred for 1 h. The insoluble material was filtered and the evaporation of solvent, followed by flash chromatography $($ EtOAc/Hex $=1 / 6)$ of the resulting residue gave $577 \mathrm{mg}(37 \%)$ of an oil. 48: ${ }^{1} \mathrm{H}$ NMR (500 MHz, $\left.\mathrm{CDCl}_{3}\right)$ : $\square 1.48-1.62$ (m, 4H), 1.70-1.78 (m, H), 1.80-1.88 (m, H), 3.48-3.53 (m, H), 3.82-3.89 (m, H), 4.00-4.07 (m, H), 4.19-4.25 (m, H), 4.68 (t, J = 3.6 Hz, H), 4.73-4.80 (m, 
2H), 5.20-5.30 (m, H); ${ }^{13} \mathrm{C}$ NMR (125 MHz, $\left.\mathrm{CDCl}_{3}\right) \square 19.8,25.8,31.0,62.6,65.2,76.1,88.2,98.1$, 209.7.

(b) Addition to 48. To a solution of $\mathrm{Pd}(\mathrm{PhCN})_{2} \mathrm{Cl}_{2}(3.8 \mathrm{mg}, 0.010 \mathrm{mmol} \mathrm{Pd}), \mathrm{P}\left(\mathrm{C}_{6} \mathrm{~F}_{5}\right)_{3}(10.6 \mathrm{mg}$, $0.020 \mathrm{mmol}$ ), and $\mathrm{Me}_{3} \mathrm{Sn}_{-} \mathrm{SnMe}_{3}(72.6 \mathrm{mg}, 0.220 \mathrm{mmol})$ in $1 \mathrm{~mL}$ of $\mathrm{C}_{6} \mathrm{D}_{6}$ at $\mathrm{rt}$ was added allenyne 48 (30.8 $\mathrm{mg}, 0.200 \mathrm{mmol})$ and the reaction mixture was kept in an NMR tube at rt. Progress of the reaction was followed by ${ }^{1} \mathrm{H}$ NMR. After $12 \mathrm{~h}$ at rt, the starting material completely converted into a mixture of $\mathrm{E} / \mathrm{Z}$ mixture of product (ratio of $\mathrm{E} / \mathrm{Z}=2.4 / 1.0$ ). Solvent was evaporated from the contents of the NMR tube and the residual oil was purified by flash chromatography (Hex/Et $3 \mathrm{~N}=$ 95/5) to get $94 \mathrm{mg}(97 \%)$ to get a mixture of $(E)$ and $(Z)-(\mathbf{4 9})$ as a colorless oil. (E/Z) ${ }^{1} \mathrm{H}$ NMR (400 MHz, $\mathrm{CDCl}_{3}$ ) $\square 0.08$ (s, 9H, Z-isomer), 0.10 (s, 9H, E-isomer), 0.11 (s, 9H, E-isomer), 0.18 (s, 9H, Z-isomer), 1.46-1.90 (m, 6H, from THP groups of both $E$ and $\mathrm{Z}$ isomers), 1.95-2.20 (m, 2H, from $\mathrm{CH}_{2} \mathrm{SnMe}_{3}$ of both $E$ and $\mathrm{Z}$ isomers), 3.50 (m, $E / Z$ ), 3.87 (m, $E / Z$ ), 4.01 (m, $E$-isomer), 4.20 (m, $E / Z$ ), 4.62 (m, E/Z), 5.46 (t br, $E$-isomer), 5.97 (t br, $Z$-isomer); ${ }^{13} \mathrm{C} \mathrm{NMR} \mathrm{(100} \mathrm{MHz,} \mathrm{CDCl}_{3}$ ): mixture of $\mathrm{E} / \mathrm{Z}$ isomers $\mathrm{C}-9.74,-9.68,-9.2,-8.3,19.42,19.45,24.3,25.4,30.6,30.7,62.05,62.15$, $63.7,68.9,97.4,98.0,130.2,130.5,130.7,146.6,148.9$.

Conversion of $1 \mathrm{a}$ to an acyclic allylstannanes 25. This reaction carried out using trimethylsilyltributylstannane at room temperature has already been discussed under cyclization studies (see above).

\section{Reaction of 7 with $\mathrm{Ph}_{3} \mathrm{Sn}-\mathrm{SiMe}_{3}$ and $\mathrm{n}-\mathrm{Bu}_{3} \mathrm{Sn}-\mathrm{SiMe}_{3}$ to get 1-(2-(trimethylsilyl)hept-1-en-4-yl)-} 5-(prop-2-ynyl)pyrrolidin-2-one 63 (Table 4, Entries 6 and 7 and Eq. 16). In a two-necked flask equipped with a reflux condenser, stirring bar, rubber septum and a $\mathrm{N}_{2}$ inlet were placed $\mathrm{Pd}_{2}(\mathrm{dba})_{3} \cdot \mathrm{CHCl}_{3}(0.125 \mathrm{mmol}, 129 \mathrm{mg})$ and $\mathrm{P}\left(\mathrm{C}_{6} \mathrm{~F}_{5}\right)_{3}(0.25 \mathrm{mmol}, 133 \mathrm{mg})$. A solution of allenyne $7(2.5 \mathrm{mmol}, 543 \mathrm{~g})$ in $20 \mathrm{~mL}$ of benzene followed by $1.088 \mathrm{~g}$ of $\mathrm{Me}_{3} \mathrm{Si}_{-}-\mathrm{SnBu}_{3}-\mathrm{n}$ or $1.266 \mathrm{~g}$ of $\mathrm{Me}_{3} \mathrm{Si}_{-} \mathrm{SnPh}_{3}(3 \mathrm{mmol})$ were added. The rubber septum was quickly replaced with a glass stopper and the dark mixture was heated at $45^{\circ} \mathrm{C}$ for $48 \mathrm{~h}$. Analysis by TLC indicated the completion of the reaction showing a non-polar spot. The reaction mixture was concentrated and passed through a short column of $\mathrm{SiO}_{2}$ eluting with $10 \%$ EtOAc in hexanes containing a few drops of $\mathrm{Et}_{3} \mathrm{~N}$, to get product 51 from $\mathrm{Me}_{3} \mathrm{Si}_{-} \mathrm{SnBu}_{3}-\mathrm{n}$ reaction $(912 \mathrm{mg}, 63 \%)$ and $\mathbf{5 2}$ from $\mathrm{Me}_{3} \mathrm{Si}_{-} \mathrm{SnPh}_{3}$ reaction (1.198 g, 75\%) as sticky materials. Individual treatment of $\mathbf{5 1}$ and $\mathbf{5 2}(1 \mathrm{mmol})$ with CSA (4 mmol, 928 $\mathrm{mg}$ ) in $20 \mathrm{~mL}$ of $\mathrm{CH}_{3} \mathrm{CN}$ and $0.5 \mathrm{~mL}$ of water followed by work up as in the case of $\mathbf{3 2 / 3 3}$ (vide 
supra, eq 10) afforded a crude material which was purified by flash column chromatography (15\% EtOAc in hexanes) to get diastereomeric mixture of $\mathbf{6 3}$ (128 mg, 44\%) as an oil: ${ }^{1} \mathrm{H}$ NMR (500 $\mathrm{MHz}) \square 5.6(\mathrm{t}, \mathrm{J}=1.5 \mathrm{~Hz}, 1 \mathrm{H}), 5.51(\mathrm{~d}, \mathrm{~J}=2 \mathrm{~Hz}, 1 \mathrm{H}$ from minor isomer), $5.4(\mathrm{~d}, \mathrm{~J}=2.85 \mathrm{~Hz}, 1 \mathrm{H})$, $5.37(\mathrm{t}, \mathrm{J}=1.25 \mathrm{~Hz}, 1 \mathrm{H}$ from minor isomer), 4.12-4.06 and 3.75-3.59 $(2 \mathrm{~m}, 2 \mathrm{H}), 2.68(\mathrm{dd}, \mathrm{J}=13.5$, $6.5 \mathrm{~Hz}, 1 \mathrm{H}), 2.57-2.12(\mathrm{~m}, 6 \mathrm{H}), 1.2(\mathrm{t}, \mathrm{J}=1.5 \mathrm{~Hz}, 1 \mathrm{H}), 1.99-1.93(\mathrm{~m}, 1 \mathrm{H}), 1.69-1.62(\mathrm{~m}, 2 \mathrm{H}), 1.37-$ $1.2(\mathrm{~m}, 2 \mathrm{H}), 0.9(\mathrm{t}, \mathrm{J}=7 \mathrm{~Hz}, 3 \mathrm{H}$ from minor isomer), $0.89(\mathrm{t}, \mathrm{J}=7 \mathrm{~Hz}, 3 \mathrm{H}), 0.11$ and $0.09(2 \mathrm{~s}, 9 \mathrm{H}$ from each isomer); ${ }^{13} \mathrm{C}$ NMR (125 MHz) $\square 175.9$ and 175.7, 149.5 and 148.9, 127.5 and 125.9, 80.5 and 80.4, 71.3 and 71.2, 57.4 and 56.4, 53.4 and 51.7, 40.3and 33.6, 37.7 and 37.1, 30.7 and 30.6, 25.8 and 25.5, 24.8 and 24.7, 20.5 and 20.5, 14.4 and 14.3, -0.9 and -1.2.

Table 4, entry 8 ((E)-1-(Tributylstannyl)-4-(tetrahydro-2H-pyran-2-yloxy)but-2-en-2yl)trimethylsilane (53). To a solution of $\mathrm{Pd}\left(\mathrm{PhCN}_{2} \mathrm{Cl}_{2}(3.8 \mathrm{mg}, 0.010 \mathrm{mmol} \mathrm{Pd}), \mathrm{P}\left(\mathrm{C}_{6} \mathrm{~F}_{5}\right)_{3}(11 \mathrm{mg}\right.$, $0.020 \mathrm{mmol}$ ), and $\mathrm{Bu}_{3} \mathrm{SnSiMe}_{3}$ (79.9 mg, $0.220 \mathrm{mmol}$ ) in $1 \mathrm{~mL}$ of $\mathrm{C}_{6} \mathrm{D}_{6}$ at rt was added allene 48 (30.8 $\mathrm{mg}, 0.200 \mathrm{mmol}$ ) and the reaction mixture was kept in an NMR tube at rt. After $12 \mathrm{~h}$ at rt, the ${ }^{1} \mathrm{H}$ NMR spectrum indicated a clean conversion into the desired product $\mathbf{5 3}$ and less than $5 \%$ of unidentified isomeric product. Solvent was evaporated from the contents of the NMR tube and the residual oil was purified by flash chromatography $\left(\mathrm{Hex} / \mathrm{Et}_{3} \mathrm{~N}=95 / 5\right)$ to get $95.5 \mathrm{mg}(92 \%)$ of product as a colorless oil. 53: IR (thin film): 2956, 2874, 2853, 1641, 1518, 1484, 1464, 1377, 1342, 1292, $1247 \mathrm{~cm}^{-1}$; ${ }^{1} \mathrm{H}$ NMR (500 MHz, $\mathrm{CDCl}_{3}$ ): $\square$ (peaks below 3.0): 3.43-3.53 (m, H), 3.88 (ddd, $J=3.1,7.9,11.3 \mathrm{~Hz}, \mathrm{H}), 4.01(\mathrm{dd}, J=6.3,13.2 \mathrm{~Hz}, \mathrm{H}), 4.24(\mathrm{dd}, J=5.0,13.2 \mathrm{~Hz}, \mathrm{H}), 4.62$ $(\mathrm{t}, J=3.8 \mathrm{~Hz}, 1 \mathrm{H}), 5.58\left(\mathrm{t}, J=5.7 \mathrm{~Hz}, 1 \mathrm{H},{ }^{4} J_{\mathrm{Sn}-\mathrm{H}}=188 \mathrm{~Hz}, \mathrm{H}\right)$; See Figure 5 for nOe data. ${ }^{13} \mathrm{C}$ NMR (125 MHz, $\left.\mathrm{CDCl}_{3}\right)$ : $\square-1.8,9.9,12.9,13.5,19.5,25.4,27.3,29.0,30.5,62.1,64.7,98.1$, 129.6, 143.3: HRMS calcd for $\left[(\mathrm{M}+\mathrm{Na})^{+}\right] 541.2500$, found 541.2492. Selected nOe contacts confirm the assigned double bond geometry.

Distannylation with $\mathrm{Me}_{3} \mathrm{SnSnMe}_{3}$ gave the corresponding E- and Z- products (E/Z)Trimethyl(1-(trimethylstannyl)-4-(tetrahydro-2H-pyran-2-yloxy)but-2-en-2-yl)stannane (49) in a ratio of 2.4:1.0 (Table 3, entry 6). See Supporting Information for details.

\section{Synthesis of 3-Propa-1,2-dienyl-4-(tert-butyldimethylsilanyloxymethyl)-oxazolidin-2-one (55)}

(Scheme 9). The allene $\mathbf{5 5}$ was obtained as a side product as a result of base promoted isomerization of corresponding propargyl derivatives (Scheme 9). To a stirred suspension of $\mathrm{NaH}$ (864 mg, $36.0 \mathrm{mmol})$ in THF (100 mL) was added the starting alcohol, 2-carbobenzyloxyamino-1O-tert-butyldimethylsiloxypropan-3-ol $(\mathbf{5 8}, 5.09 \mathrm{~g}, 15.0 \mathrm{mmol})$ in THF $(10 \mathrm{~mL})$ dropwise. The 
reaction mixture was stirred at $\mathrm{rt}$ for $15 \mathrm{~h}$ and propargyl bromide $(3.83 \mathrm{~mL}, 45.0 \mathrm{mmol})$ in THF (30 $\mathrm{mL}$ ) was added to the mixture. The reaction was quenched followed by normal extractive workup gave a residue which was purified by flash chromatography (EtOAc/Hex/acetone =1/2/1) to get 4(tert-butyldimethylsilanyloxymethyl)-3-prop-2-ynyl-oxazolidin-2-one (54) (30\%) and $\mathbf{5 5}$ (46\%) as pale yellow oils. 55: IR (thin film): 3036, 2954, 2929, 2957, 2884, 2710, 1963, $1766 \mathrm{~cm}^{-1}$; ${ }^{1} \mathrm{H}$ NMR (500 MHz, $\mathrm{CDCl}_{3}$ ): $\square 0.05$ (s, 6H), 0.88 (s, 9H), $3.66\left(\mathrm{dd}, \mathrm{J}=2.6,10.6 \mathrm{~Hz}, \mathrm{H}, \mathrm{CH}_{2} \mathrm{OSi}\right), 3.81$ (dd, J = 5.0, $10.6 \mathrm{~Hz}, \mathrm{H}, \mathrm{CH}_{2} \mathrm{OSi}$ ), 3.92 (m, H, CH CH $\mathrm{CHSi}_{2}, 4.38$ (d, J = 3.0 Hz, H, $\mathrm{CH}_{2} \mathrm{CH}$ ), 4.39 $\left(\mathrm{s}, \mathrm{H}, \mathrm{CH}_{2} \mathrm{CH}\right), 5.39$ (dd, J = 6.5, $\left.10.0 \mathrm{~Hz}, \mathrm{H}, \mathrm{NCH}=\mathrm{C}=\mathrm{CH}_{2}\right), 5.44(\mathrm{dd}, \mathrm{J}=6.5,10.0 \mathrm{~Hz}$, $\left.\mathrm{NCH}=\mathrm{C}=\mathrm{CH}_{2}\right), 6.85\left(\mathrm{t}, \mathrm{J}=6.5 \mathrm{~Hz}, \mathrm{NCH}=\mathrm{C}=\mathrm{CH}_{2}\right) ;{ }^{13} \mathrm{C} \mathrm{NMR}\left(125 \mathrm{MHz}, \mathrm{CDCl}_{3}\right) \square-6.5,-5.0,18.5$, 26.1, 56.1, 61.0, 65.7, 88.1, 96.5, 155.8, 201.5; HRMS calcd for $[\mathrm{M}+\mathrm{H}]^{=\bullet} 270.1525$, found 270.1526.

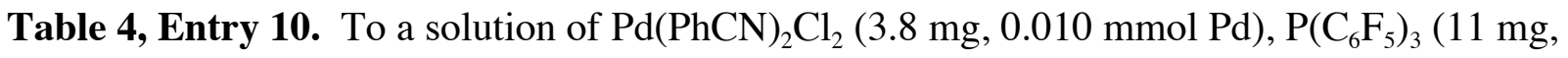
$0.020 \mathrm{mmol})$, and $\mathrm{Bu}_{3} \mathrm{SnSiMe}_{3}(79.9 \mathrm{mg}, 0.220 \mathrm{mmol})$ in $1 \mathrm{~mL}$ of $\mathrm{C}_{6} \mathrm{D}_{6}$ at $\mathrm{rt}$ was added allene 55 (53.9 $\mathrm{mg}, 0.200 \mathrm{mmol}$ ) and the reaction mixture was kept in an NMR tube at $\mathrm{rt}$. After $12 \mathrm{~h}$ at $\mathrm{rt}$, the ${ }^{1} \mathrm{H}$ NMR spectrum indicated a clean conversion into the desired product $\mathbf{5 7}$ and less than $1 \%$ of isomeric product, which could not be identified. Solvent was evaporated from the contents of the NMR tube and the residual oil was purified by flash chromatography $\left(\mathrm{Hex}_{2} / \mathrm{Et}_{3} \mathrm{~N}=95 / 5\right)$ to get 59 mg (47\%) of product 57 as an oil. 57: IR (thin film): 2955, 2927, 1754, 1607, 1464, 1402, 1378, 1342, 1249, 1170, 1128, 1076, 1056, 1006, 909, 838, 802, 778, $734 \mathrm{~cm}^{-1}$; ${ }^{1} \mathrm{H}$ NMR (500 MHz, $\mathrm{CDCl}_{3}$ ): $\square 0.03$ ( s, 3H, $\mathrm{SiCH}_{3}$ ), 0.04 (s, 3H, $\mathrm{SiCH}_{3}$ ), 0.10 (s, 9H, SiCH $H_{3}, 0.87$ (s, 9H, t-Bu), 1.70 (d, $\left.J=12.0 \mathrm{~Hz}, \mathrm{H}, \mathrm{CH}_{2} \mathrm{Sn}\right), 1.76\left(\mathrm{~d}, J=12.0 \mathrm{~Hz}, \mathrm{H}, \mathrm{CH}_{2} \mathrm{Sn}\right), 3.52(\mathrm{dd}, J=2.2,10.4 \mathrm{~Hz}, \mathrm{H}$, $\mathrm{CH}_{2} \mathrm{OTBS}$ ), 3.64 (dd, $J=4.7,10.4 \mathrm{~Hz}, \mathrm{H}, \mathrm{CH}_{2} \mathrm{OTBS}$ ), 4.13-4.18 (m, H, OCH${ }_{2} \mathrm{CH}$ ), 4.28-4.37 (m, $\left.2 \mathrm{H}, \mathrm{OCH}_{2} \mathrm{CH}\right), 5.86\left(\mathrm{~s}, J_{\mathrm{Sn}-\mathrm{H}}=20 \mathrm{~Hz}, \mathrm{H}, \mathrm{CH}=\mathrm{C}\right) ;{ }^{13} \mathrm{C} \mathrm{NMR}\left(125 \mathrm{MHz}, \mathrm{CDCl}_{3}\right)$ : $\square-5.12,-5.09,-1.1$, $10.8\left(J_{\mathrm{Sn}-\mathrm{C}}=312 \mathrm{~Hz}\right), 14.1,18.5,26.1,27.8\left(J_{\mathrm{Sn}-\mathrm{C}}=58 \mathrm{~Hz}\right), 29.6\left(J_{\mathrm{Sn}-\mathrm{C}}=20 \mathrm{~Hz}\right), 57.3,61.5,65.4$, 122.6, 132.9, 157.3; HRMS calcd for [M+Na] 656.2953, found 656.2964. A selected nOe contact confirms the double bond geometry depicted (See Figure 1).

\section{Synthesis and silylstannylation of $(E)$-Ethyl 3-((S)-3-(buta-2,3-dienyl)-2-oxooxazolidin-4-} yl)acrylate (60) (Scheme 9). The TBS ether 59 (666 mg, $2.35 \mathrm{mmol})$ was dissolved in THF (5 mL) at $0{ }^{\circ} \mathrm{C}$ and a solution of TBAF $(1.0 \mathrm{M}$ in THF, $2.58 \mathrm{~mL}, 2.58 \mathrm{mmol})$ was added dropwise. The reaction was complete in $1 \mathrm{~h}$ as judged by TLC (EtOAc/Hex $\left.=3 / 1, \mathrm{R}_{\mathrm{f}}=0.25\right)$. Silica gel chromatography of the residue after evaporation gave $345 \mathrm{mg}(87 \%)$ of allenyl alcohol 9. 9: IR 
(neat, $\mathrm{NaCl}): 3411,2985,2931,1957,1731,1479,1445,1361 \mathrm{~cm}^{-1} ;{ }^{1} \mathrm{H}$ NMR $\left(500 \mathrm{MHz}, \mathrm{CDCl}_{3}\right)$ : 2.64 (s br, H, OH), 3.60-3.68 (m, H, $\left.\mathrm{CH}_{2} \mathrm{OH}\right), 3.72-3.78$ (m, H, NCH$)_{2}, 3.80$ (d br, J = 11.9 Hz, H, $\left.\mathrm{CH}_{2} \mathrm{OH}\right), 3.90-3.96(\mathrm{~m}, \mathrm{H}, \mathrm{CH}), 4.05-4.09$ (m, H, NCH$)_{2}, 4.26$ (dd, $\left.\mathrm{J}=6.0,8.6 \mathrm{~Hz}, \mathrm{H}, \mathrm{OCH}_{2} \mathrm{CH}\right)$, $4.35\left(\mathrm{t}, J=8.8 \mathrm{~Hz}, \mathrm{H}, \mathrm{OCH}_{2} \mathrm{CH}\right.$ ), 4.85 (t br, $J=3.2 \mathrm{~Hz}, \mathrm{H}, \mathrm{C}=\mathrm{CH}_{2}$ ), 5.15 (quintet, $J=6.7 \mathrm{~Hz}, \mathrm{H}$, $\mathrm{CH}=\mathrm{C}) ;{ }^{13} \mathrm{C} \mathrm{NMR}\left(125 \mathrm{MHz}, \mathrm{CDCl}_{3}\right): \square 41.6,56.6,61.0,64.9,77.6,86.3,158.9,209.4$; HRMS calcd for [M+Na] 192.0631, found 192.0613.

To a solution of DMSO $(0.18 \mathrm{~mL}, 2.5 \mathrm{mmol})$ in $2 \mathrm{~mL}$ of $\mathrm{CH}_{2} \mathrm{Cl}_{2}$ was added $(\mathrm{COCl})_{2}(0.11 \mathrm{~mL}$, $1.2 \mathrm{mmol})$ dropwise at $-60{ }^{\circ} \mathrm{C}$. After $5 \mathrm{~min}$, a solution of alcohol $9^{5}(178 \mathrm{mg}, 1.05 \mathrm{mmol})$ in $2 \mathrm{~mL}$ of $\mathrm{CH}_{2} \mathrm{Cl}_{2}$ was added dropwise over $8 \mathrm{~min}$. After stirring $15 \mathrm{~min}$ at $-60{ }^{\circ} \mathrm{C}, \mathrm{Et}_{3} \mathrm{~N}(0.59 \mathrm{~mL}, 4.2 \mathrm{mmol})$ was added and the mixture was warmed slowly to $-15^{\circ} \mathrm{C}$ over $20 \mathrm{~min}$ and the crude mixture was used in the next step.

To a reaction mixture at $-15^{\circ} \mathrm{C}$ above was added a solution of carbethoxymethylenetriphenylphosphorane (733 mg, $2.10 \mathrm{mmol}$ ) in $2 \mathrm{~mL}$ of $\mathrm{CH}_{2} \mathrm{Cl}_{2}$ and the temperature was allowed to reach $\mathrm{rt}$ over $1.5 \mathrm{~h}$. The reaction mixture was poured into brine $(20 \mathrm{~mL})$ and the aqueous layer was extracted with ether $\left(20 \mathrm{~mL}\right.$ x 4). The combined organic layer was dried $\left(\mathrm{MgSO}_{4}\right)$, evaporated, and the residue was subjected to flash chromatography $($ EtOAc/Hex $=1 / 2)$ to get $202 \mathrm{mg}(81 \%)$ of $\mathbf{6 0}$ as an oil. ${ }^{1} \mathrm{H}$ and ${ }^{13} \mathrm{C}$ NMR indicated that the isolated material was a single double bond isomer. 60. IR (neat, $\mathrm{NaCl}) 3063,2983,2909,2260,1956,1764,1731,1714,1660,1651 \mathrm{~cm}^{-1} ;{ }^{1} \mathrm{H}$ NMR $(500$ $\mathrm{MHz}, \mathrm{CDCl}_{3}$ ): $\square 1.29$ (t, $J=7.1 \mathrm{~Hz}, 3 \mathrm{H}, \mathrm{OCH}_{2} \mathrm{CH}_{3}$ ), $3.53\left(\mathrm{dd}, J=7.5,15.3 \mathrm{~Hz}, \mathrm{H}, \mathrm{NCH}_{2}\right), 3.96-$ 4.05 (m, H, CH), 4.10 (dd br, $\left.J=3.4,15.3 \mathrm{~Hz}, \mathrm{H}, \mathrm{NCH}_{2}\right), 4.21$ (q, $J=7.1 \mathrm{~Hz}, 2 \mathrm{H}, \mathrm{OCH}_{2} \mathrm{CH}_{3}$ ), 4.45 $\left(\mathrm{d}, J=4.6 \mathrm{~Hz}, 2 \mathrm{H}, \mathrm{OCH}_{2} \mathrm{CH}\right), 4.77-4.86(\mathrm{~m}, 2 \mathrm{H}$, allene), 5.07 (quintet, $J=6.6 \mathrm{~Hz}, \mathrm{H}$, allene), 6.03 $(\mathrm{d}, J=15.6 \mathrm{~Hz}, \mathrm{H}, \mathrm{C}=\mathrm{CH}), 6.72(\mathrm{dd}, J=7.0,15.6 \mathrm{~Hz}, \mathrm{H}, \mathrm{C}=\mathrm{CH}) ;{ }^{13} \mathrm{C} \mathrm{NMR}\left(125 \mathrm{MHz}, \mathrm{CDCl}_{3}\right)$ : 14.6, 41.6, 56.7 , 61.4, 66.7, 77.6, 85.6, 126.8, 142.6, 157.8, 165.4, 209.8; HRMS calcd for [M+Na] 260.0899, found 260.0905.

\section{(2E)-Ethyl 3-((S)-3-((E)-4-(tributylstannyl)-3-(trimethylsilyl)but-2-enyl)2-oxooxazolidin-4-}

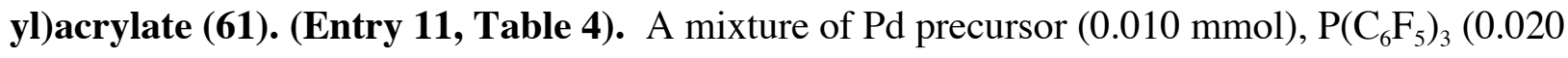
$\mathrm{mmol})$, Sn-Si reagent $(0.22 \mathrm{mmol})$, and eneallene $60(0.20 \mathrm{mmol})$ in $\mathrm{C}_{6} \mathrm{D}_{6}(1 \mathrm{~mL})$ was prepared and the reaction was followed by NMR. After the reaction was complete, the reaction mixture was concentrated in vacuo and was subjected to silica gel column chromatography $\left(\mathrm{Et}_{3} \mathrm{~N} / \mathrm{Hex}=5 / 95\right)$ to get the corresponding product. After column chromatography, $84 \%$ of $\mathbf{6 1}$ was obtained as an oil. The isolated material was a single double-bond isomer $\left({ }^{1} \mathrm{H},{ }^{13} \mathrm{C} \mathrm{NMR}\right)$. 61. IR (neat, $\left.\mathrm{NaCl}\right) 2956$, 
2924, 1766, 1728, 1660, 1416, 1373, 1351, $1300 \mathrm{~cm}^{-1}$; ${ }^{1} \mathrm{H}$ NMR (500 MHz, $\left.\mathrm{CDCl}_{3}\right): \square 0.04$ (s, 9H), $\mathrm{SiMe}_{3}, \mathrm{SnBu}_{3}$ peaks omitted, 1.12-1.30 (t, 3H, CO $\left.2 \mathrm{Et}\right), 1.77\left(\mathrm{~d}, J=11.7 \mathrm{~Hz}, \mathrm{H}, J_{\mathrm{Sn}-\mathrm{H}}=64 \mathrm{~Hz}, \mathrm{H}\right.$, $\left.\mathrm{CH}_{2} \mathrm{Sn}\right), 1.83\left(\mathrm{~d}, J=11.7 \mathrm{~Hz}, J_{\mathrm{Sn}-\mathrm{H}}=64 \mathrm{~Hz}, \mathrm{H}, \mathrm{CH}_{2} \mathrm{Sn}\right), 3.64\left(\mathrm{dd}, J=7.3,15.6 \mathrm{~Hz}, \mathrm{H}, \mathrm{NCH}_{2}\right), 3.94$ $\left(\mathrm{dd}, J=5.4,15.6 \mathrm{~Hz}, \mathrm{H}, \mathrm{NCH}_{2}\right), 4.00$ (dd, $\left.J=7.6,8.7 \mathrm{~Hz}, \mathrm{H}, \mathrm{OCH}_{2}\right), 4.15-4.27$ (m, 2H, $\left.\mathrm{CO}_{2} \mathrm{Et}\right)$, $4.33(\mathrm{dd}, J=8.6,12.5 \mathrm{~Hz}, \mathrm{H}, \mathrm{NCH}), 4.45\left(\mathrm{t}, J=8.7 \mathrm{~Hz}, \mathrm{H}, \mathrm{OCH}_{2}\right), 5.28$ (t, $J=6.6 \mathrm{~Hz}, \mathrm{H}$, $\mathrm{CH}=\mathrm{C}(\mathrm{Si})), 5.98\left(\mathrm{~d} \mathrm{br}, J=15.6 \mathrm{~Hz}, \mathrm{H}, \mathrm{C}=\mathrm{CHCO}_{2} \mathrm{Et}\right), 6.76(\mathrm{dd}, J=8.5,15.6 \mathrm{~Hz}, \mathrm{H}$, $\left.\mathrm{CH}=\mathrm{CHCO}_{2} \mathrm{Et}\right)$; See Figure 5 for nOe data. ${ }^{13} \mathrm{C} \mathrm{NMR}\left(125 \mathrm{MHz}, \mathrm{CDCl}_{3}\right): \square-1.2,10.5,13.6,14.1$, 14.6, 27.8, 29.5, 41.3 , 57.1, 61.4, 66.7, 125.6, 126.2, 143.4, 147.7, 158.1, 165.3; HRMS calcd for $\mathrm{C}_{27} \mathrm{H}_{51} \mathrm{NO}_{4} \mathrm{SiSnNa},[\mathrm{M}+\mathrm{Na}]^{=}$624.2502, found 624.2477.

\section{Reaction of $\square$-lactam 8a with trimethylsilyltributylstannane. [3R(1'R,4R)-3-[1-(tert-}

Butyldimethylsilyloxy)ethyl]-1-N-(2-propynyl)-4-(3-tri- $n$-butylstannyl-2-trimethylsilyl-1propenyl)-azetidinone (62a) (entry 12, Table 4). To a solution of [3R(1'R, 4R)-3-[1-(tertButyldimethylsilyloxy)ethyl]-4-(1,2-propadienyl)-1-N-(2-propynyl)-azetidinone (16 mg, 0.05 mmol) in $1 \mathrm{~mL} \mathrm{C}_{6} \mathrm{D}_{6}$ were added $\mathrm{Bu}_{3} \mathrm{SnSiMe}_{3}\left(18 \square \mathrm{L}, 0.05 \mathrm{mmol}, 1\right.$ equiv.), $\mathrm{Pd}_{2}(\mathrm{dba})_{3}(1 \mathrm{mg}, 0.001$ mmol, $2 \mathrm{~mol} \%)$ and $\mathrm{P}\left(\mathrm{C}_{6} \mathrm{~F}_{5}\right)_{3}(3 \mathrm{mg}, 0.005 \mathrm{mmol}, 10 \mathrm{~mol} \%)$. The reaction was monitored by ${ }^{1} \mathrm{H}$ NMR. After 15 minutes at room temperature, the reaction was complete (100\% yield by NMR). The solvent was evaporated and the crude mixture purified by column chromatography $\left(\mathrm{SiO}_{2}\right.$ : hexanes / diethyl ether 5/1) to give $8 \mathrm{mg}$ (23\% isolated yield) of 62a as a colorless oil. ${ }^{1} \mathrm{H}$ NMR $\left(500 \mathrm{MHz}, \mathrm{C}_{6} \mathrm{D}_{6}\right): \square 5.47\left(\mathrm{~d}, \mathrm{~J}=9.4,1 \mathrm{H}, \mathrm{CH}^{4} \mathrm{CH}\right), 4.62\left(\mathrm{dd}, \mathrm{J}=9.4,2.1 \mathrm{~Hz}, 1 \mathrm{H}, H^{4}\right), 4.20$ (m, 1 $\left.\mathrm{H}, H^{3}\right), 4.00\left(\mathrm{dd}, \mathrm{J}=17.9,2.5 \mathrm{~Hz}, 1 \mathrm{H}, \mathrm{NCH}_{2}\right), 3.56\left(\mathrm{dd}, \mathrm{J}=17.9 \mathrm{~Hz}, 2.5,1 \mathrm{H}, \mathrm{NCH}_{2}\right), 2.70(\mathrm{t}, \mathrm{J}=$ $2.4 \mathrm{~Hz}, 1 \mathrm{H}, \mathrm{CHOSi}), 2.17\left(\mathrm{dd}, \mathrm{J}_{\mathrm{Sn}-\mathrm{H}}=38.3, \mathrm{~J}=11.7 \mathrm{~Hz}, 2 \mathrm{H}, \mathrm{SnCH}_{2} \mathrm{CSi}\right.$ ), 1.84 (t, J = 2.5 Hz, $1 \mathrm{H}$, $\left.\mathrm{NCH}_{2} \mathrm{CCH}\right), 1.63\left(\mathrm{~m}, 6 \mathrm{H}, \mathrm{SnCH}_{2}\right), 1.40\left(\mathrm{~m}, 6 \mathrm{H}, \mathrm{SnCH}_{2} \mathrm{CH}_{2}\right), 1.08\left(\mathrm{~m}, 6+3 \mathrm{H}, \mathrm{Sn}\left(\mathrm{CH}_{2}\right)_{2} \mathrm{CH}_{2}+\right.$ $\left.\mathrm{CH}_{3}\right), 1.03$ (s, $\left.9 \mathrm{H}, \mathrm{OSi} B u\right), 0.96\left(\mathrm{t}, \mathrm{J}=7.3 \mathrm{~Hz}, 9 \mathrm{H}, \mathrm{Sn}\left(\mathrm{CH}_{2}\right)_{3} \mathrm{CH}_{3}\right), 0.26\left(\mathrm{~s}, 3 \mathrm{H}, \mathrm{OSiCH}_{3}\right), 0.15$ (s, 9 $\mathrm{H}, \mathrm{CSiCH}_{3}$ ), $0.14\left(\mathrm{~s}, 3 \mathrm{H}, \mathrm{OSiCH}_{3}\right.$ ); nOe (See Figure 5) : $\mathrm{CH}^{4} \mathrm{CH} \square \mathrm{CHOSi}: 2 \%, \mathrm{CH}^{4} \mathrm{CH} \square$ $\mathrm{CSiCH}_{3}: 1 \% ;{ }^{13} \mathrm{C} \mathrm{NMR}\left(125 \mathrm{MHz}, \mathrm{C}_{6} \mathrm{D}_{6}\right): \square 205.5,166.5,147.5,131.0,79.8,71.6,66.6,64.8$, $50.4,29.7,29.2,27.9,26.3,22.8,18.4,14.0,13.9,10.7,-1.3,-4.2,-4.5 ;{ }^{119} \mathrm{Sn}\left(186 \mathrm{MHz}, \mathrm{C}_{6} \mathrm{D}_{6}\right) \square-$ 20.6; IR (film): $1743 \mathrm{~cm}^{-1}$; HRMS calcd for $\mathrm{C}_{32} \mathrm{H}_{63} \mathrm{NO}_{2} \mathrm{Si}_{2} \mathrm{SnNa}: \mathrm{M}^{\bullet+}=693.33117$, found 693.33396.

The same reaction mixture left for longer reaction time $(1 \mathrm{~h})$ at room temperature or left at higher temperatures $\left(30\right.$ to $60^{\circ} \mathrm{C}$ ) for short periods of time (10-20 minutes) only deterioration of the products was observed, with no indication of cyclization. 
Silylstannylation of Eneallene 8b with Trimethylsilyltributylstannane. [3R(1'R,4R)-3-[1-(tertButyldimethylsilyloxy)ethyl]-1-N-(2-propynyl)-4-(3-tri-n-butylstannyl-2-trimethylsilyl-1propenyl)-azetidinone (Table 4, Entry 12$)$. To a solution of [3R(1'R,4R)-3-[1-(tertButyldimethylsilyloxy)ethyl]-4-(1,2-propadienyl)-1-N-(2-propynyl)-azetidinone (8b) (25 mg, 0.08 mmol) in $1 \mathrm{~mL} \mathrm{C}_{6} \mathrm{D}_{6}$ were added $\mathrm{Bu}_{3} \mathrm{SnSiMe}_{3}$ (29 $\square \mathrm{L}, 0.08 \mathrm{mmol}, 1$ equiv.), $\mathrm{Pd}_{2}(\mathrm{dba})_{3}(2 \mathrm{mg}, 0.001$ mmol, $2 \mathrm{~mol} \%)$ and $\mathrm{P}\left(\mathrm{C}_{6} \mathrm{~F}_{5}\right)_{3}(5 \mathrm{mg}, 0.008 \mathrm{mmol}, 10 \mathrm{~mol} \%)$. The reaction was monitored by ${ }^{1} \mathrm{H}$ NMR. After 15 minutes at room temperature, the reaction was complete (100\% yield by NMR). The solvent was evaporated and the crude mixture purified by column chromatography $\left(\mathrm{SiO}_{2}\right.$ : hexanes / diethyl ether 5/1) to give $45 \mathrm{mg}$ (82\% isolated yield) of $\mathbf{6 2 b}$ as a colorless oil. ${ }^{1} \mathrm{H}$ NMR (500 MHz, $\left.\mathrm{C}_{6} \mathrm{D}_{6}\right): \square 5.76\left(\mathrm{~m}, 1 \mathrm{H}, \mathrm{NCH}_{2} \mathrm{CH}\right), 5.44$ (d, J = 9.4, $\left.1 \mathrm{H}, \mathrm{CH}^{4} \mathrm{CH}\right), 5.20$ (ddd, J = 15.8, 2.9, 1.4, $\left.1 \mathrm{H}, \mathrm{NCH}_{2} \mathrm{CHCH}_{2}\right), 5.03\left(\mathrm{dd}, \mathrm{J}=10.2,1.4,1 \mathrm{H}, \mathrm{NCH}_{2} \mathrm{CHCH}_{2}\right), 4.52(\mathrm{dd}, \mathrm{J}=9.4,2.1,1 \mathrm{H}$, $\left.H^{4}\right), 4.25(\mathrm{dq}, \mathrm{J}=6.2,2.4, \mathrm{CHOSi}), 3.92\left(\mathrm{ddt}, \mathrm{J}=16.1,5.1,1.5,1 \mathrm{H}, \mathrm{NCH}_{2}\right), 3.53(\mathrm{dd}, \mathrm{J}=16.1,6.0$, $\left.1 \mathrm{H}, \mathrm{NCH}_{2}\right), 2.74\left(\mathrm{t}, \mathrm{J}=2.3,1 \mathrm{H}, H^{3}\right), 2.07\left(\mathrm{dd}, \mathrm{J}_{\mathrm{Sn}-\mathrm{H}}=38.3, \mathrm{~J}=11.7,2 \mathrm{H}, \mathrm{SnCH}_{2} \mathrm{CSi}\right), 1.59(\mathrm{~m}, 6$ $\left.\mathrm{H}, \mathrm{SnCH}_{2}\right), 1.38\left(\mathrm{~m}, 6 \mathrm{H}, \mathrm{SnCH}_{2} \mathrm{CH}_{2}\right.$ ), 1.08 (d, J = 6.3, $3 \mathrm{H}, \mathrm{CH}_{3}$ ), 1.02 (s, $9 \mathrm{H}, \mathrm{OSiBu}$ overlapping m, $\left.6 \mathrm{H}, \mathrm{Sn}\left(\mathrm{CH}_{2}\right)_{2} \mathrm{CH}_{2}\right), 0.94\left(\mathrm{t}, \mathrm{J}=7.4,9 \mathrm{H}, \mathrm{Sn}\left(\mathrm{CH}_{2}\right)_{3} \mathrm{CH}_{3}\right), 0.24\left(\mathrm{~s}, 3 \mathrm{H}, \mathrm{OSiCH}_{3}\right), 0.14(\mathrm{~s}, 3 \mathrm{H}$, $\mathrm{OSiCH}_{3}$ ), 0.12 (s, $9 \mathrm{H}, \mathrm{CSiCH}_{3}$ ); nOe : $\mathrm{CH}^{4} \mathrm{CH} \square \mathrm{CHOSi}: 3 \%, \mathrm{CH}^{4} \mathrm{CH} \square \mathrm{CSiCH}_{3}: 2 \%$; ${ }^{13} \mathrm{C}$ NMR (125 MHz, $\left.\mathrm{C}_{6} \mathrm{D}_{6}\right)$ : \206.0, 167.1, 147.2, 134.0, 131.6, 116.9, 66.2, 64.8, 50.2, 43.1, 29.7, $29.6,29.5,27.8,26.2,22.9,18.4,13.9,13.8,10.7,-1.4,-4.4,-4.4 ;{ }^{119} \mathrm{Sn}\left(186 \mathrm{MHz}, \mathrm{C}_{6} \mathrm{D}_{6}\right) \square-20.3$; IR (film): $1759 \mathrm{~cm}^{-1}$; HRMS calcd for $\mathrm{C}_{32} \mathrm{H}_{65} \mathrm{NO}_{2} \mathrm{Si}_{2} \mathrm{SnNa}$ : [M] = 694.34682, found 694.34175. Cyclization of 61. Synthesis of Piperidines (Eq 17). To a refluxing solution of allyltin compound $61(56 \mathrm{mg}, 0.0938 \mathrm{mmol})$ in benzene $\mathrm{C}_{6} \mathrm{H}_{6}(9 \mathrm{~mL})$ was added via syringe pump a mixture of AIBN $(3.1 \mathrm{mg})$ and $\mathrm{Bu}_{3} \mathrm{SnH}(55 \mathrm{mg}, 0.1875 \mathrm{mmol})$ over $3 \mathrm{~h}$. The reaction mixture was concentrated in vacuo and the residue was subjected to silica gel chromatography $\left(\mathrm{Et}_{3} \mathrm{~N} / \mathrm{Hex}=5 / 95\right)$ to get $80 \mathrm{mg}$ (90\%) of 64 as a colorless oil. Inspection of ${ }^{1} \mathrm{H}$ and ${ }^{13} \mathrm{C}$ NMR indicated that this is a single diastereomer with $\sim 5 \%$ of a contaminant whose structure has not been identified. All the assignments were confirmed by COSY and HMQC experiments. Extensive nOe experiments support the relative stereochemistry of the major product as depicted above. Even though the bestfit structure is shown above, the configuration of the ring-carbon with the trialkyltin group should be considered tentative, because of the uncertainties involved in the nOe experiments. 64: $[\square]_{\mathrm{D}}=$ +15.0 (c $2.3 \mathrm{CH}_{2} \mathrm{Cl}_{2}$ ); IR (neat, $\left.\mathrm{NaCl}\right)$ 1760, 1728, $1455 \mathrm{~cm}^{-1} ;{ }^{1} \mathrm{H} \mathrm{NMR}\left(500 \mathrm{MHz}, \mathrm{CDCl}_{3}\right): \square 0.10$ (s, 9H), 0.80-0.95 (m, $15 \mathrm{H}), 1.21\left(\mathrm{t}, J=7.2 \mathrm{~Hz}, 3 \mathrm{H}, \mathrm{CO}_{2} \mathrm{Et}\right), 1.35-1.45$ (m, $\left.6 \mathrm{H}\right), 1.35-1.50$ (m, 7 
H) [including $1.42(\mathrm{~m}, \mathrm{H}, \mathrm{SnCH})], 2.50\left(\mathrm{ddd}, J=4.1,8.5,8.5 \mathrm{~Hz}, \mathrm{H}, \mathrm{CHC}(\mathrm{Si})=\mathrm{CH}_{2}\right), 2.98(\mathrm{dd}$ br, $J$ $\left.=4.1,4.1 \mathrm{~Hz}, 1 \mathrm{H}, \mathrm{CHCO}_{2} \mathrm{Et}\right), 3.64\left(\mathrm{~d}, J=8.5 \mathrm{~Hz}, 2 \mathrm{H}, \mathrm{NCH}_{2}\right), 3.68(\mathrm{dd}, J=6.9,8.2 \mathrm{~Hz}, 1 \mathrm{H}$, $\left.\mathrm{OCH}_{2}, \mathrm{\square}-\mathrm{H}\right), 3.96$ and $4.07\left(\mathrm{~d} \mathrm{ABq}, J=7.2,2 \mathrm{H}, \mathrm{CO}_{2} \mathrm{CH}_{2} \mathrm{CH}_{3}\right), 4.35(\mathrm{dd}, J=8.2,8.2 \mathrm{~Hz}, 1 \mathrm{H}$, $\left.\mathrm{OCH}_{2}, \square-\mathrm{H}\right), 4.51(\mathrm{ddd}, J=6.9,8.2,12.3 \mathrm{~Hz}, 1 \mathrm{H}, \mathrm{NCH}), 5.40\left(\mathrm{~d}, J=1.1 \mathrm{~Hz}, \mathrm{H}, \mathrm{C}(\mathrm{Si})=\mathrm{CH}_{2}\right.$, cis to TMS), $5.49\left(\mathrm{t}, J=1.2 \mathrm{~Hz}, \mathrm{H}, \mathrm{C}(\mathrm{Si})=\mathrm{CH}_{2}\right.$, trans to TMS); ${ }^{13} \mathrm{C} \mathrm{NMR}\left(125 \mathrm{MHz}, \mathrm{CDCl}_{3}\right) \square-0.8,9.4$, 14.0, 14.6, 27.9, 29.5, 31.5, 41.1, 43.8, 46.5, 54.1, 60.5, 68.9, 124.9, 151.0, 157.3, 173.5; HRMS calcd for [M+Na] 624.2502, found: 624.2485. 
Table . Table of Chemical Shifts and Assignments of ${ }^{1} \mathrm{H}$ NMR of 64 (Assignments were confirmed by COSY and HMQC, See Figure 1 for labels)

\begin{tabular}{|c|c|c|}
\hline chemical shift & multiplicity/coupling constant & proton \\
\hline $1.21(3 \mathrm{H})$ & $\mathrm{t}(7.2 \mathrm{~Hz})$ & $\mathrm{C}_{\mathrm{H}_{3}} \mathrm{CH}_{2} \mathrm{OC}(\mathrm{O})$ \\
\hline $1.42(-)$ & $\mathrm{m}$ & $\mathrm{H}^{\mathrm{d}}$ \\
\hline $2.50(1 \mathrm{H})$ & $\mathrm{ddd}\left(\mathrm{J}_{\mathrm{ef}}=4.1 ; \mathrm{J}_{\mathrm{fg}}=8.5 ; \mathrm{J}_{\mathrm{fh}}=8.5\right)$ & $\mathrm{H}^{\mathrm{f}}$ \\
\hline $2.98(1 \mathrm{H})$ & $\mathrm{dd}\left(\mathrm{J}_{\mathrm{de}}=4.1 ; \mathrm{J}_{\mathrm{ef}}=4.1\right)$ & $\mathrm{H}^{\mathrm{e}}$ \\
\hline $3.64(2 \mathrm{H})$ & $\mathrm{d}\left(\mathrm{J}_{\mathrm{fg}}=8.5\right)$ & $\mathrm{H}^{\mathrm{g}} / \mathrm{H}^{\mathrm{g}}$ \\
\hline $3.68(1 \mathrm{H})$ & $\mathrm{dd}\left(\mathrm{J}_{\mathrm{ab}}=8.2 ; \mathrm{J}_{\mathrm{ac}}=6.9\right)$ & \\
\hline $3.96(1 \mathrm{H})$ & $\mathrm{dABq}(\mathrm{J}=7.2)$ & $\mathrm{CH}_{3} \mathrm{CH}_{2} \mathrm{OC}(\mathrm{O})$ \\
\hline $4.07(1 \mathrm{H})$ & $\mathrm{dABq}(\mathrm{J}=7.2)$ & $\mathrm{CH}_{3} \mathrm{CH}_{2} \mathrm{OC}(\mathrm{O})$ \\
\hline $4.35(1 \mathrm{H})$ & $\mathrm{dd}\left(\mathrm{J}_{\mathrm{ab}}=8.2 ; \mathrm{J}_{\mathrm{bc}}=8.2\right)$ & \\
\hline $4.51(1 \mathrm{H})$ & $\mathrm{ddd}\left(\mathrm{J}_{\mathrm{ac}}=6.9 ; \mathrm{J}_{\mathrm{bc}}=8.2 ; \mathrm{J}_{\mathrm{cd}}=12.3\right)$ & $\mathrm{H}^{\mathrm{c}}$ \\
\hline $5.40(1 \mathrm{H})$ & $\mathrm{d}\left(\mathrm{J}_{\mathrm{k} \mathrm{k}}=1.1\right)$ & $\mathrm{H}^{\mathrm{k}}$ (cis to TMS) \\
\hline $5.49(1 \mathrm{H})$ & $\mathrm{t}(\mathrm{J}=1.1 \sim 1.2)$ & $\mathrm{H}^{\mathrm{k}^{\prime}}$ (trans to TMS) \\
\hline
\end{tabular}

\section{Summary of nOe experiments}

\begin{tabular}{ll} 
irradiate & enhancements observed \\
\hline $\mathrm{H}^{\mathrm{f}}$ & $\mathrm{H}^{\mathrm{e}}, \mathrm{H}^{\mathrm{g}}$ \\
$\mathrm{H}^{\mathrm{e}}$ & $\mathrm{H}^{\mathrm{f}}$ \\
$\mathrm{H}^{\mathrm{a}}$ & $\mathrm{H}^{\mathrm{b}} \mathrm{H}^{\mathrm{c}}$ (more than with $\mathrm{H}^{\mathrm{b}}$ ) \\
$\mathrm{H}^{\mathrm{b}}$ & $\mathrm{H}^{\mathrm{a}}, \mathrm{H}^{\mathrm{c}}$ \\
$\mathrm{H}^{\mathrm{c}}$ & $\mathrm{H}^{\mathrm{a}}, \mathrm{H}^{\mathrm{b}}$ \\
\hline
\end{tabular}




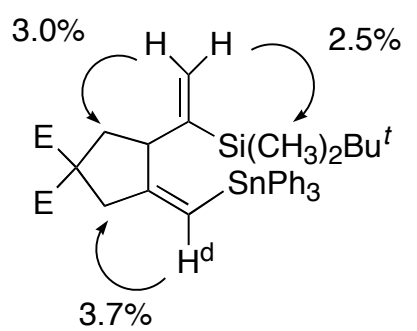

23

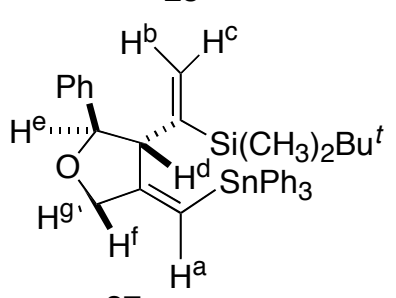

27

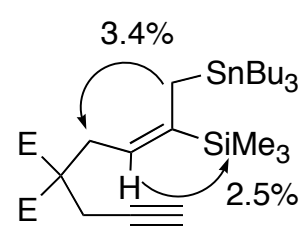

(1.8\%

25

5

$\mathrm{H}^{\mathrm{a}}-\mathrm{H}^{\mathrm{f} / \mathrm{g}} 2.4$

$\mathrm{H}^{\mathrm{d}}-\mathrm{H}^{\mathrm{e}} 1.8$

$\mathrm{H}^{\mathrm{d}}-\mathrm{Ph} 2.3$

$\mathrm{H}^{\mathrm{e}}-\mathrm{H}^{\mathrm{d}} 1.8$

$\mathrm{H}^{\mathrm{e}}-\mathrm{Bu}^{t} 2.7$

$\mathrm{H}^{\mathrm{e}}$-Me 1.8

$J_{d e}=0 \mathrm{~Hz}$

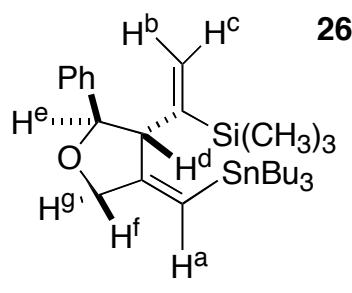

28
$\mathrm{H}^{\mathrm{a}}-\mathrm{H}^{\mathrm{f} / \mathrm{g}} 3.1$

$\mathrm{H}^{\mathrm{d}}-\mathrm{H}^{\mathrm{e}} 1.6$

$\mathrm{H}^{\mathrm{d}}-\mathrm{Ph} 2.0$

$\mathrm{H}^{\mathrm{e}}-\mathrm{H}^{\mathrm{d}} 1.4$

$\mathrm{H}^{\mathrm{b}}-\mathrm{Ph} 0$

$\mathrm{H}^{\mathrm{e}}$-Me 1.2

$J_{d e} \sim 0 \mathrm{~Hz}$

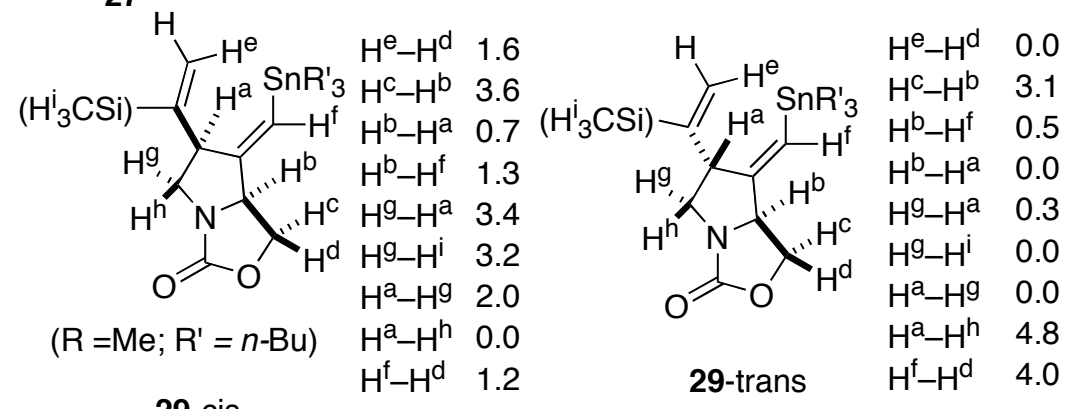

29-cis

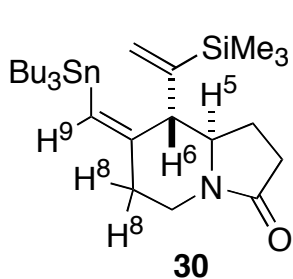

30
$J_{5,6}=8.6 \mathrm{~Hz}$

nOe: $H^{9}$ to $H^{8}(3 \%)$

$\mathrm{H}^{9}$ to $\mathrm{H}^{6}(0 \%)$

$\mathrm{H}^{5}$ to $\mathrm{H}^{6}(0 \%)$

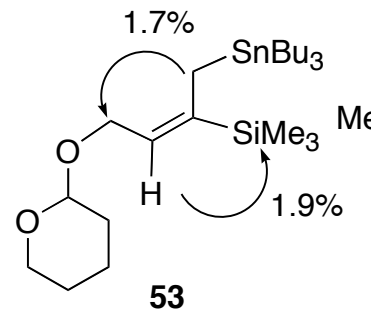

53

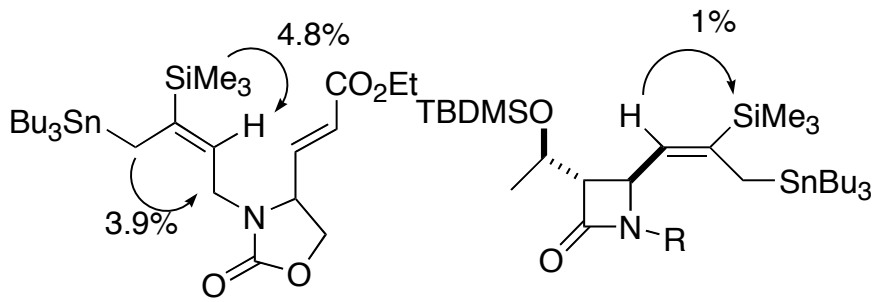

61

Figure 1. Details of nOe Experiments

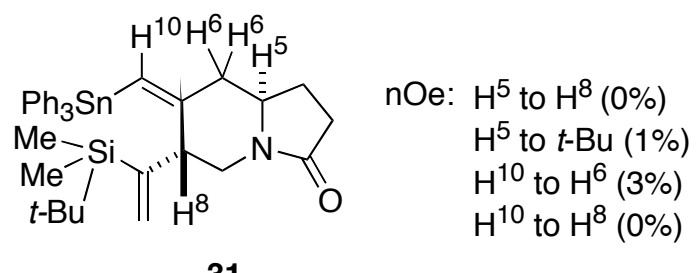

31

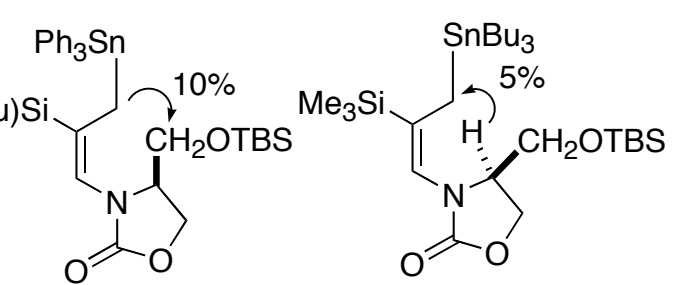

57

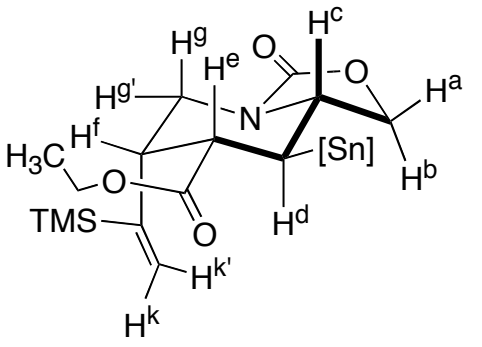

Possible conformation of the adduct 64 . $\mathrm{H}^{\mathrm{d}}-\mathrm{H}^{\mathrm{e}}$ diaxial relationship is uncertain. 


\section{References}

(1) (a) Chenard, B. L.; Laganis, E. D.; Davidson, F.; RajanBabu, T. V. J. Org. Chem. 1985, 50, 3666. (b) Warren, S.; Chow, A.; Fraenkel, G.; RajanBabu, T. V. J. Am. Chem. Soc. 2003, 125, 15402.

(2) (a) Wong, T.-T.; Busse, P. J.; Niedenzu, K. Inorg. Chem. 1970, 9, 2150. (b) Bradley, E. B.; Herber, R. H.; Busse, P. J.; Niedenzu, K. J. Organomet. Chem. 1973, 52, 297.

(3) Djahanbini, D.; Cazes, B.; Gore, J. Tetrahedron 1987, 43, 3441.

(4) Prasad, J. S.; Liebeskind, L. S. Tetrahedron Lett. 1988, 29, 4253.

(5) Chevliakov, M. V.; Montgomery, J. J. Am. Chem. Soc. 1999, 121, 11139. 
IUIAL AREE $=4 \% 5110$

MUL FACTOR=1. $0900 E+90$

* List: LIST

PEAK CAPALITY: 1243

ZERO $=0,-3.260$

ATT $2^{\wedge}=E$

CHT SP $=$ I. $E$

AP. REJ $=180 B$

THFSH $=5$

PK UL $=4.04$

* hr rej lungu

* KUN *1TIZ

JAN 8, 1902 O2:23:10

STAFT

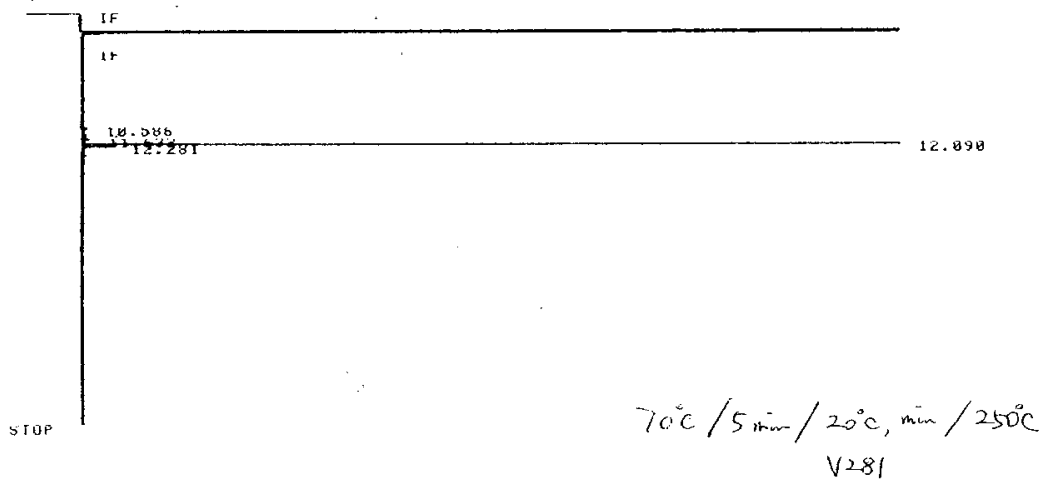

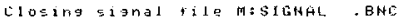

RUNA 1712 JAM 8,1902 5Z:29:10

SOENTIFIER : DCMET ZOHYS

SIGNAL FILE: $M=S$ GHAL, BHC

AREA

$\begin{array}{rrrrr}\text { RE } & \text { FREA } & \text { TYPE } & \text { WIDTH } & \text { AREA\% } \\ 12.098 & 1215799 & \text { FE } & .027 & 98.01322 \\ 12.281 & 24845 & 6 B & .018 & 1.98679\end{array}$

TOTAL AREA $=1248444$

MUL FACTOH $=1.009 E E+E B$

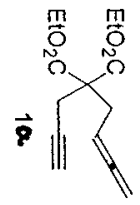

S- 


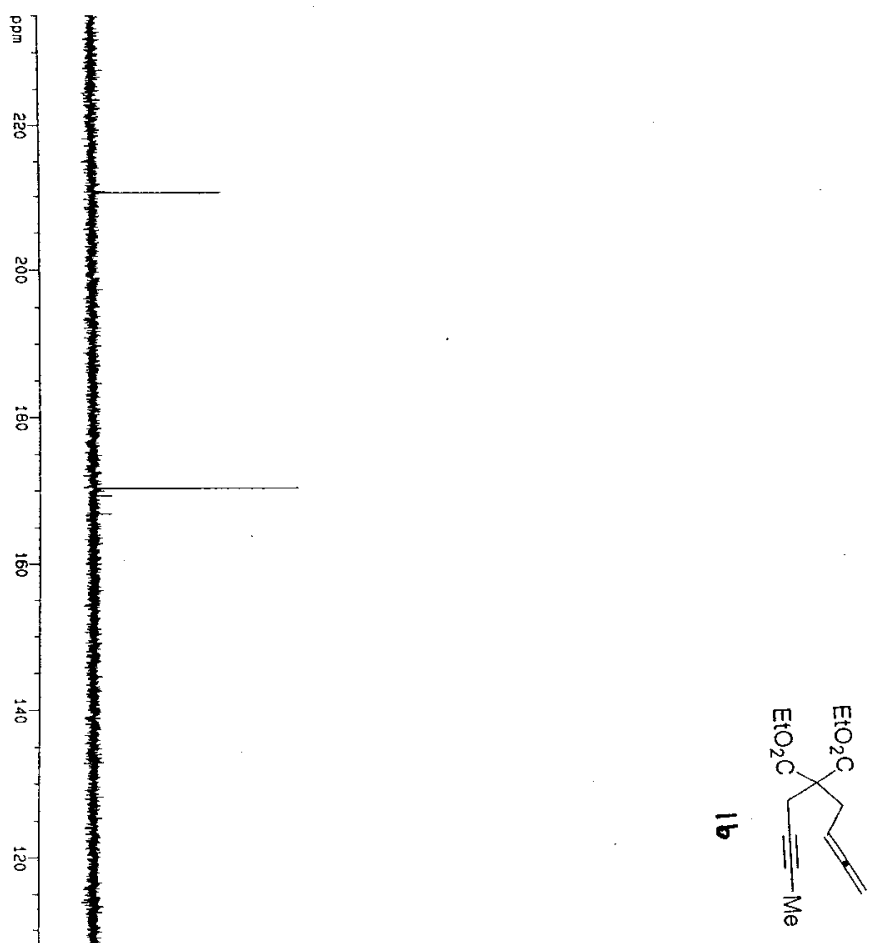

$-290.506$

$-170.313$

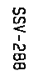
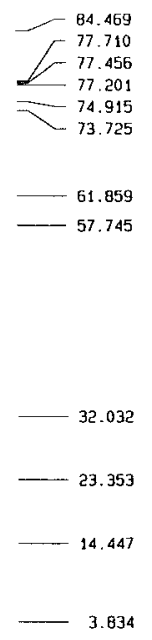

$\mathrm{S}-$
30 


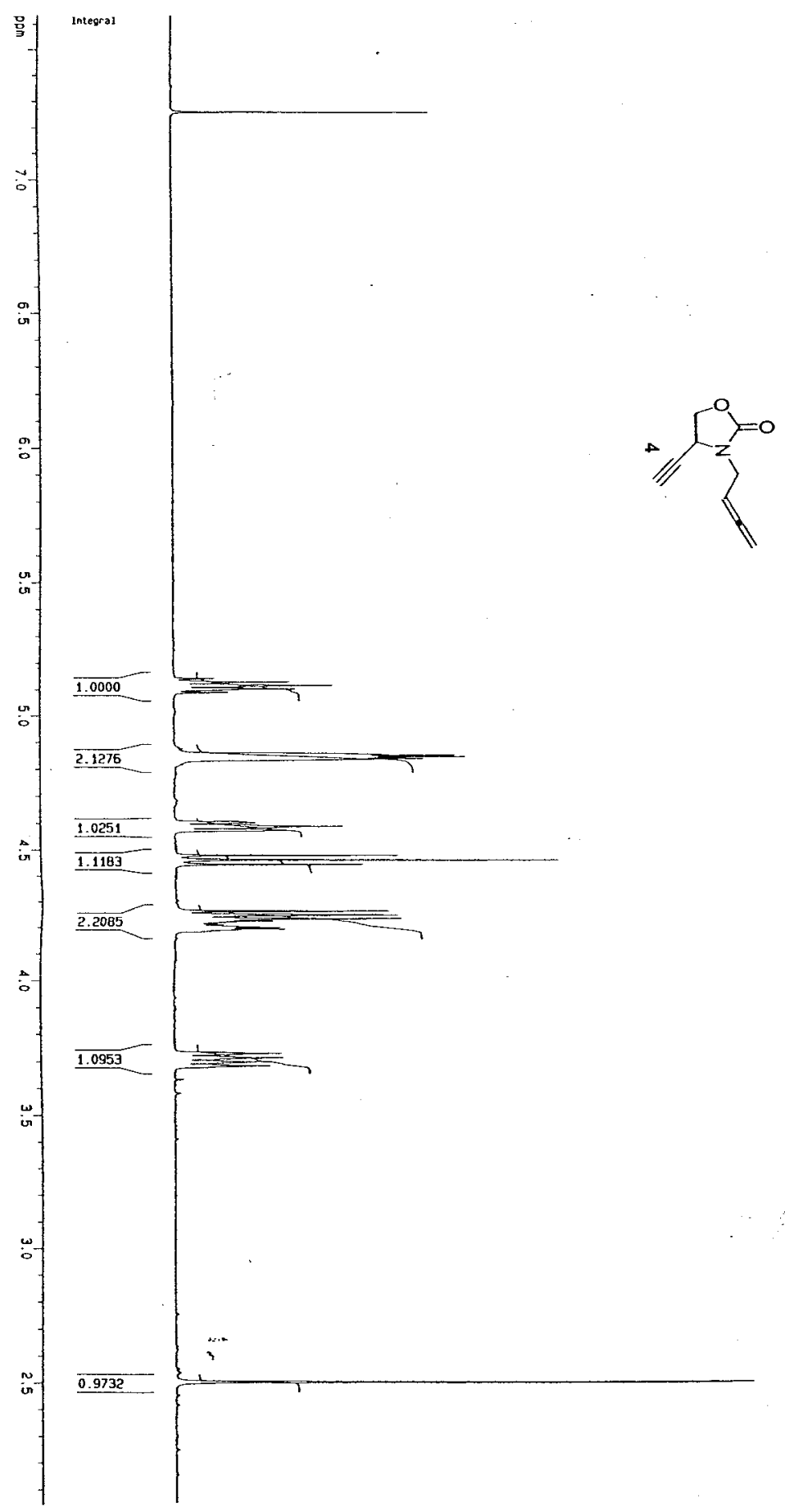

5.11797

5.11571 $-5.10504$ $-5.10272$ $-4.86073$ $-4.85882$ $\int r^{-4.85647}$ $-4.85+86$ $\int r^{-4.64958}$ $-4.84715$ 4.84568 $-4.84253$ $-4.84253$ $-483863$

4.5900 4.59080
-4.48180 $5-4.47948$

$-4.46250$

-4.46250
-4.44766

$-4.26690$

L. 4.26388

(-4.25442

4.25032

$-4.24701$

$L_{4.23750}$

$-4.23443$

$-3.73208$

$-3.72915$

$-3.71680$

3.71391
-3.70161

$-3.70161$

$-3.69866$

$-3.68632$

L3.69334 

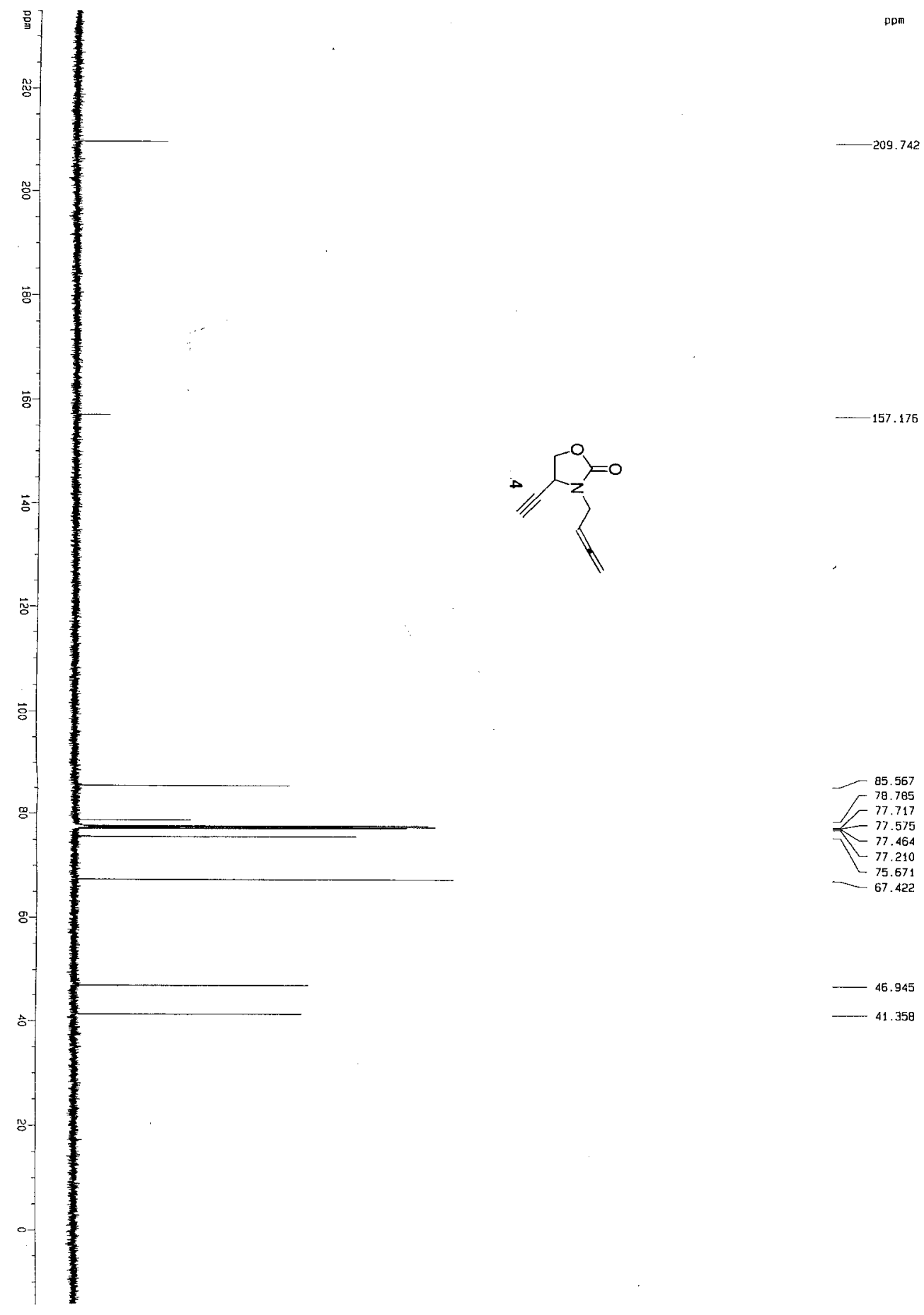

S- 


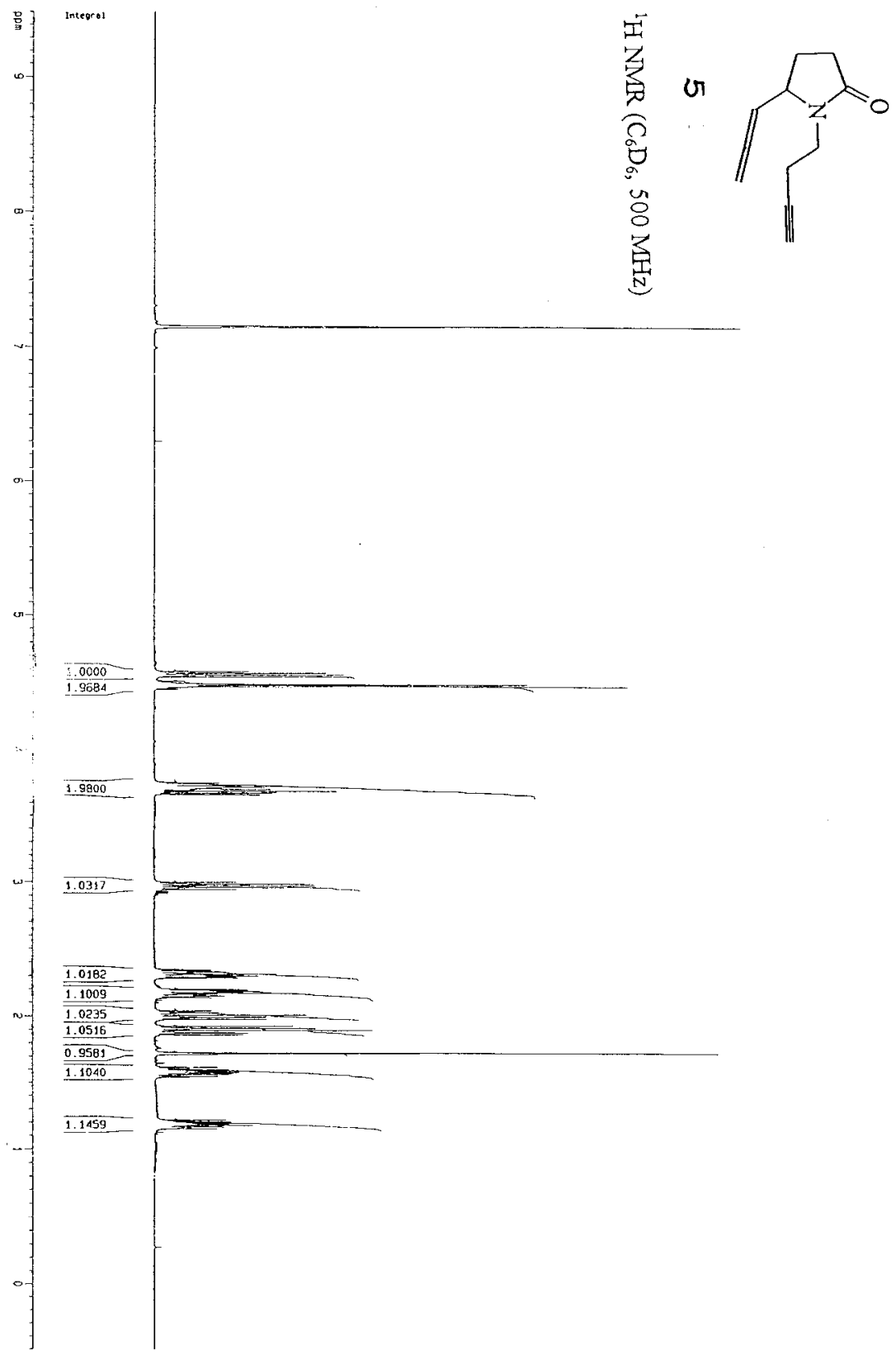

S-
33 


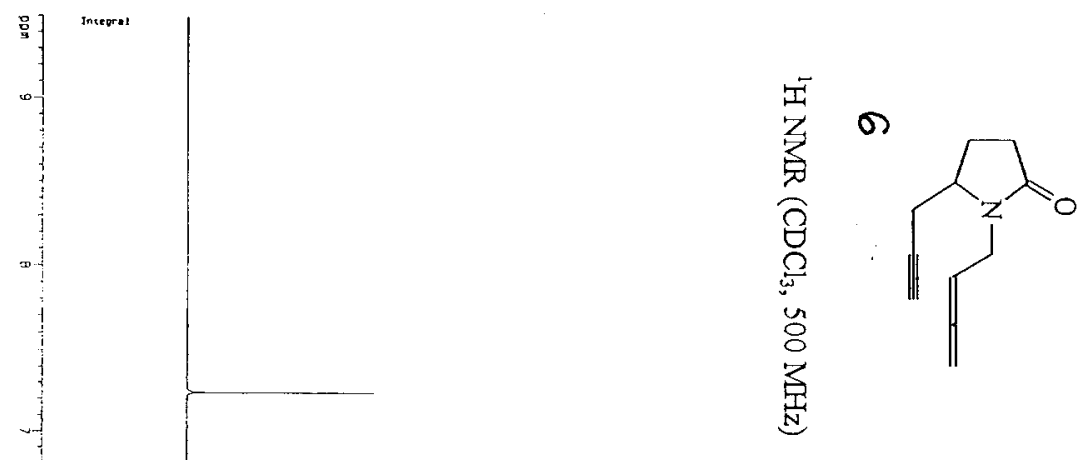

S-
34 

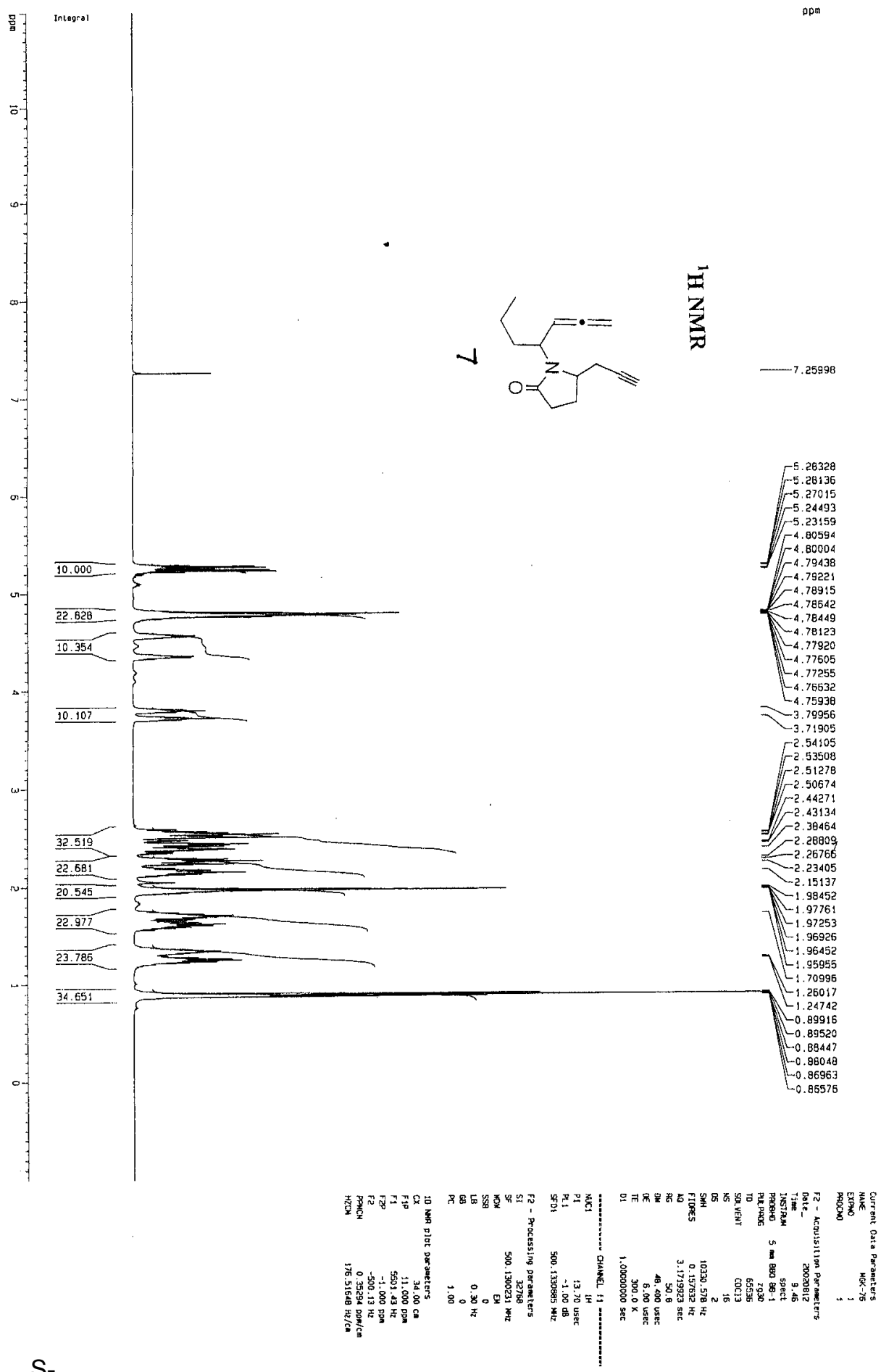

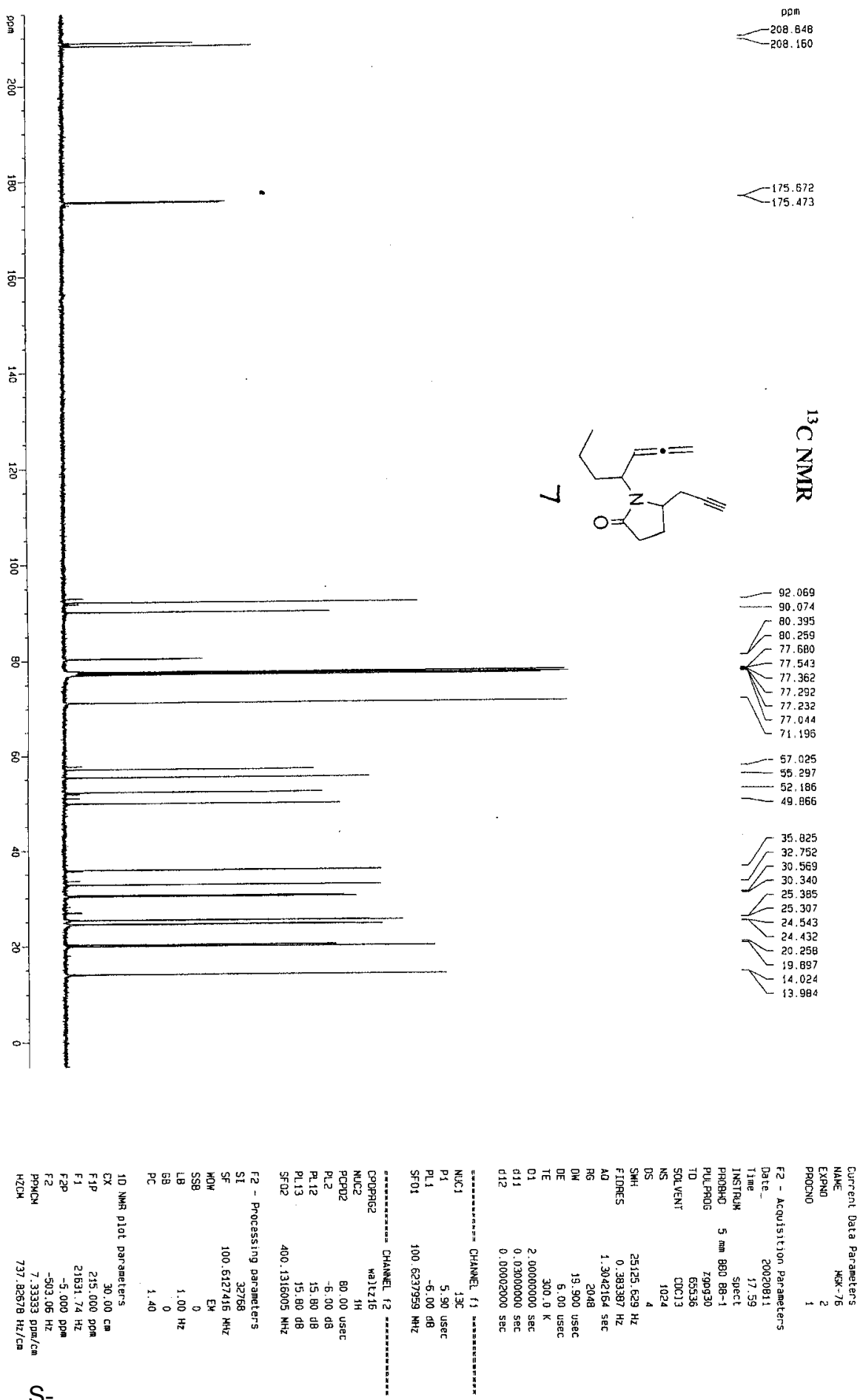


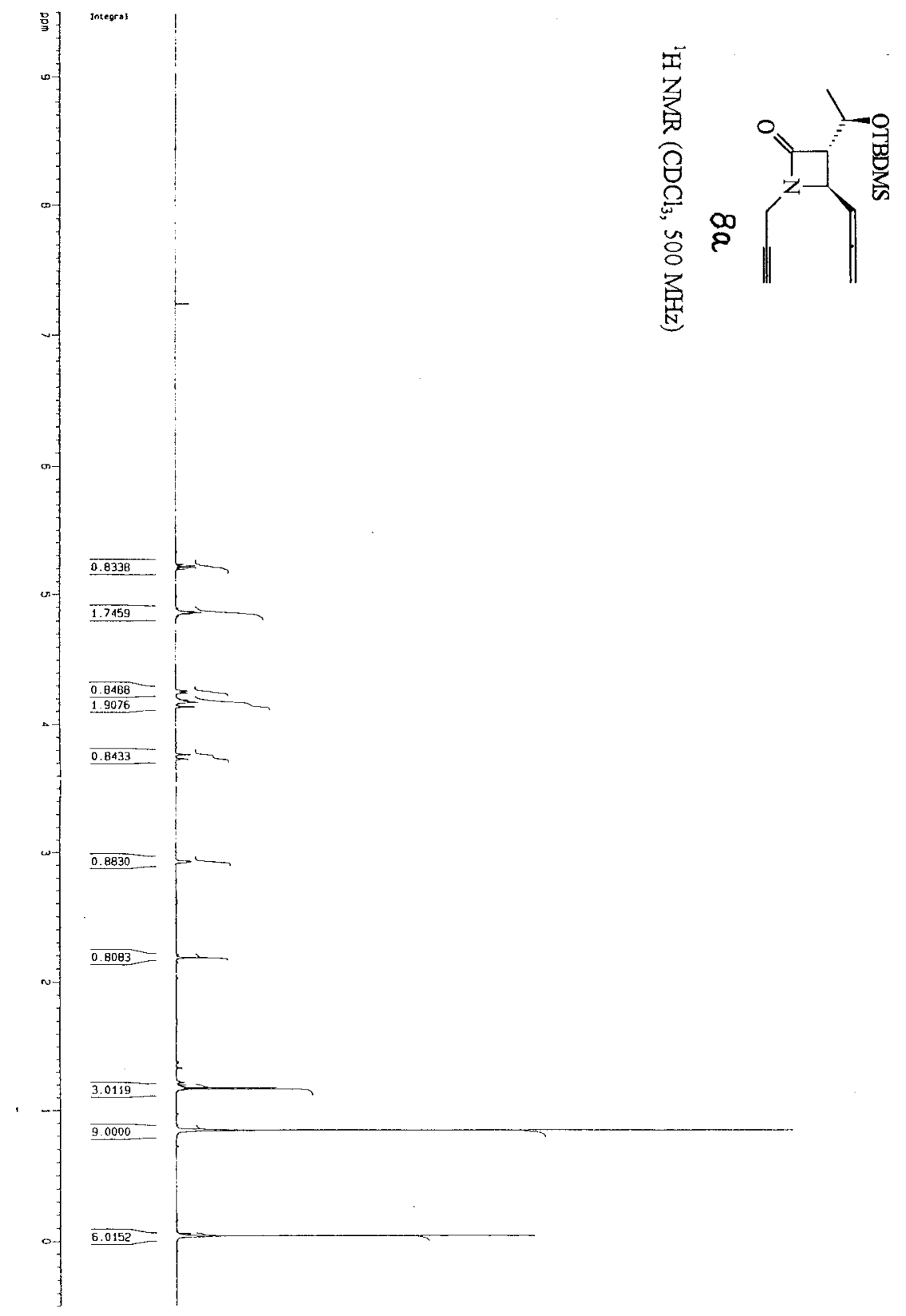

S- 


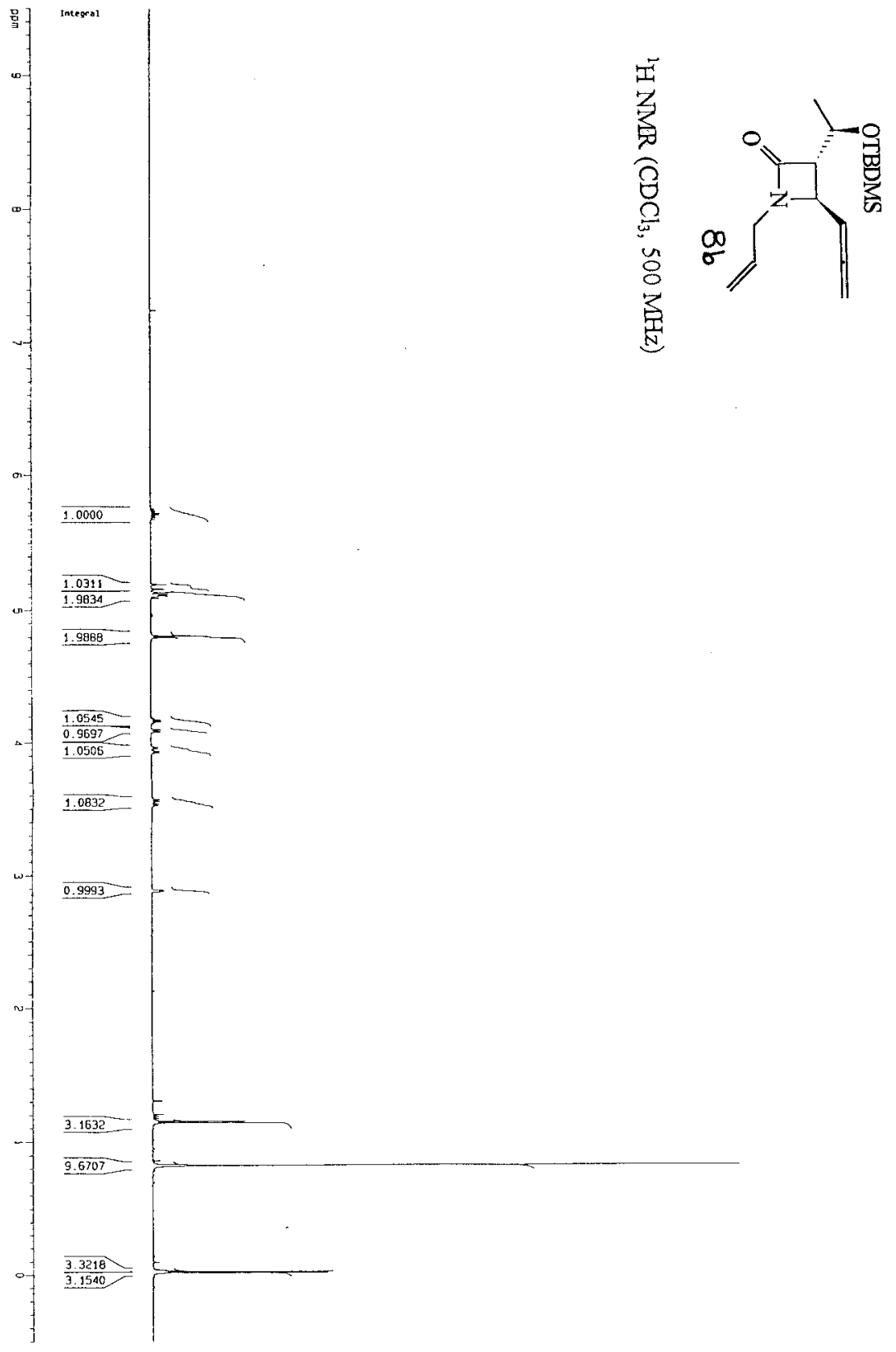

S-

38 


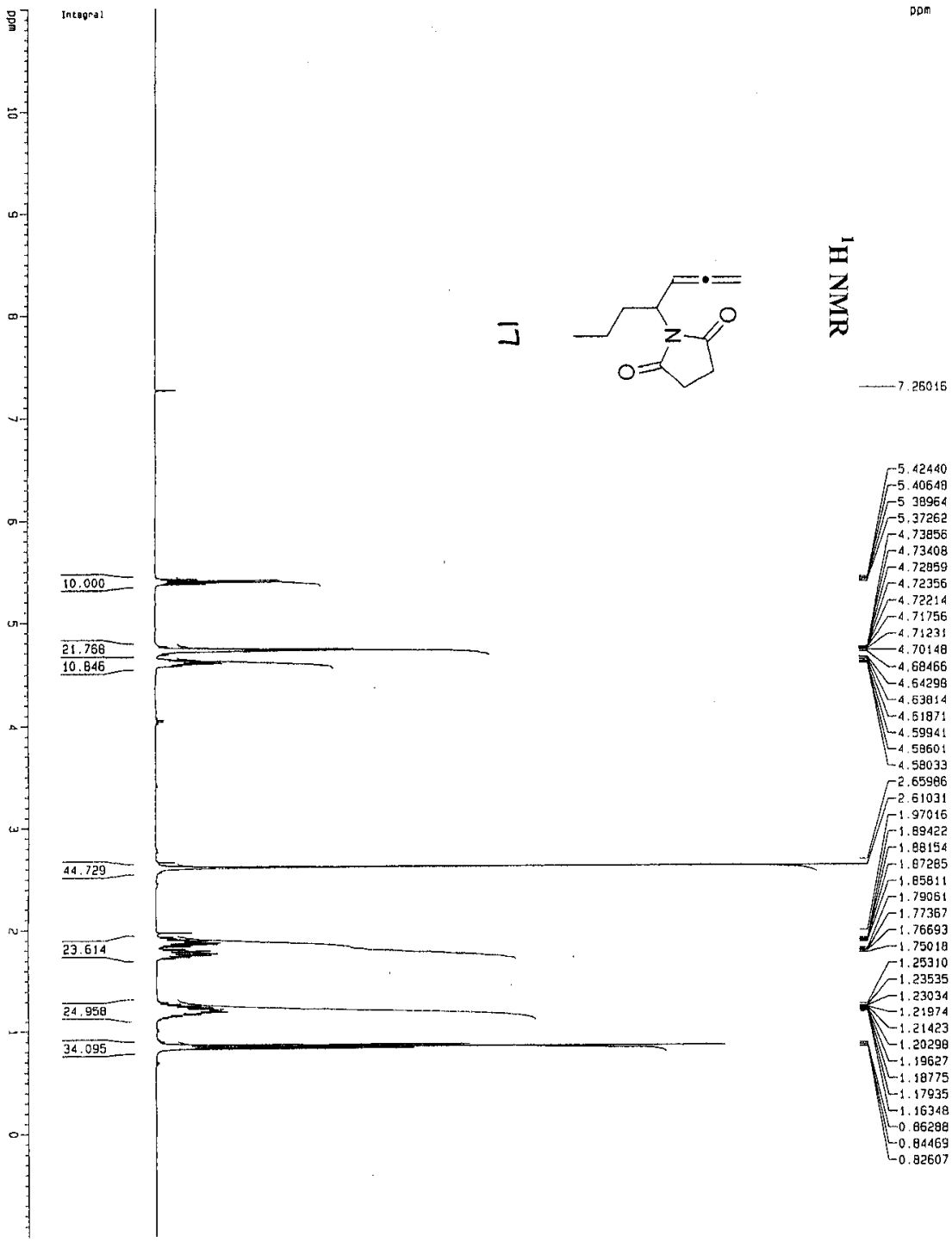

26016 

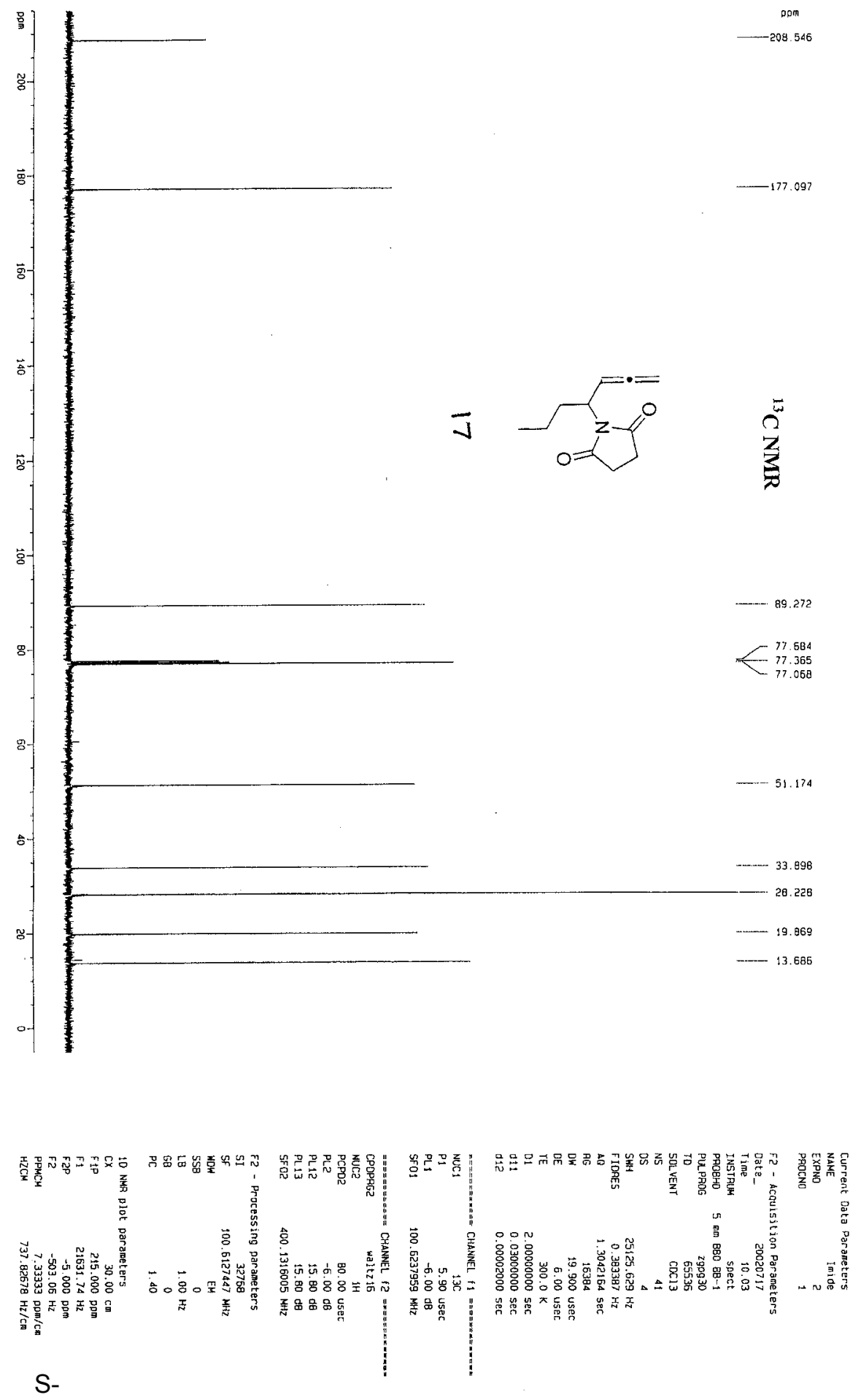

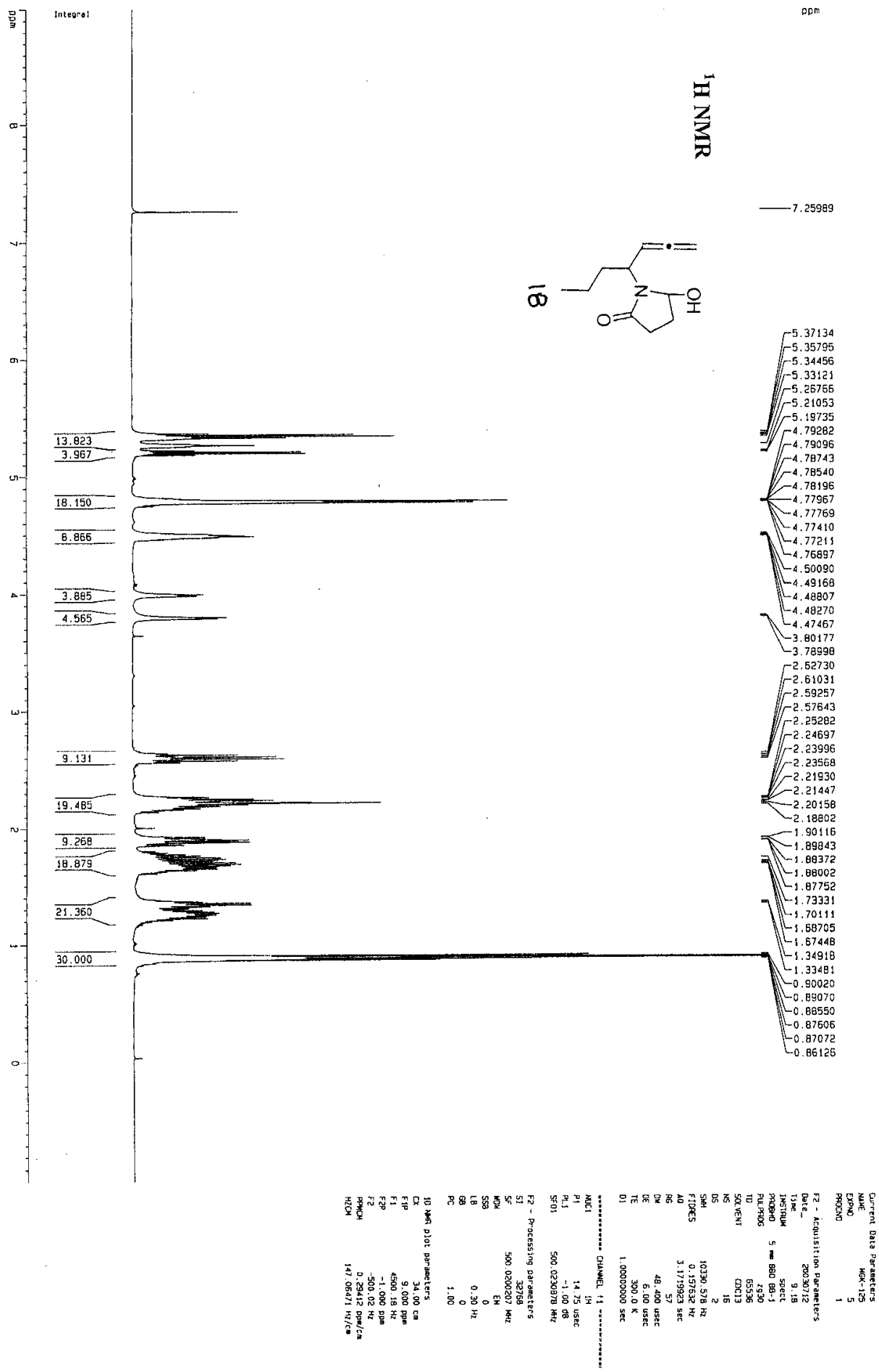

S- 

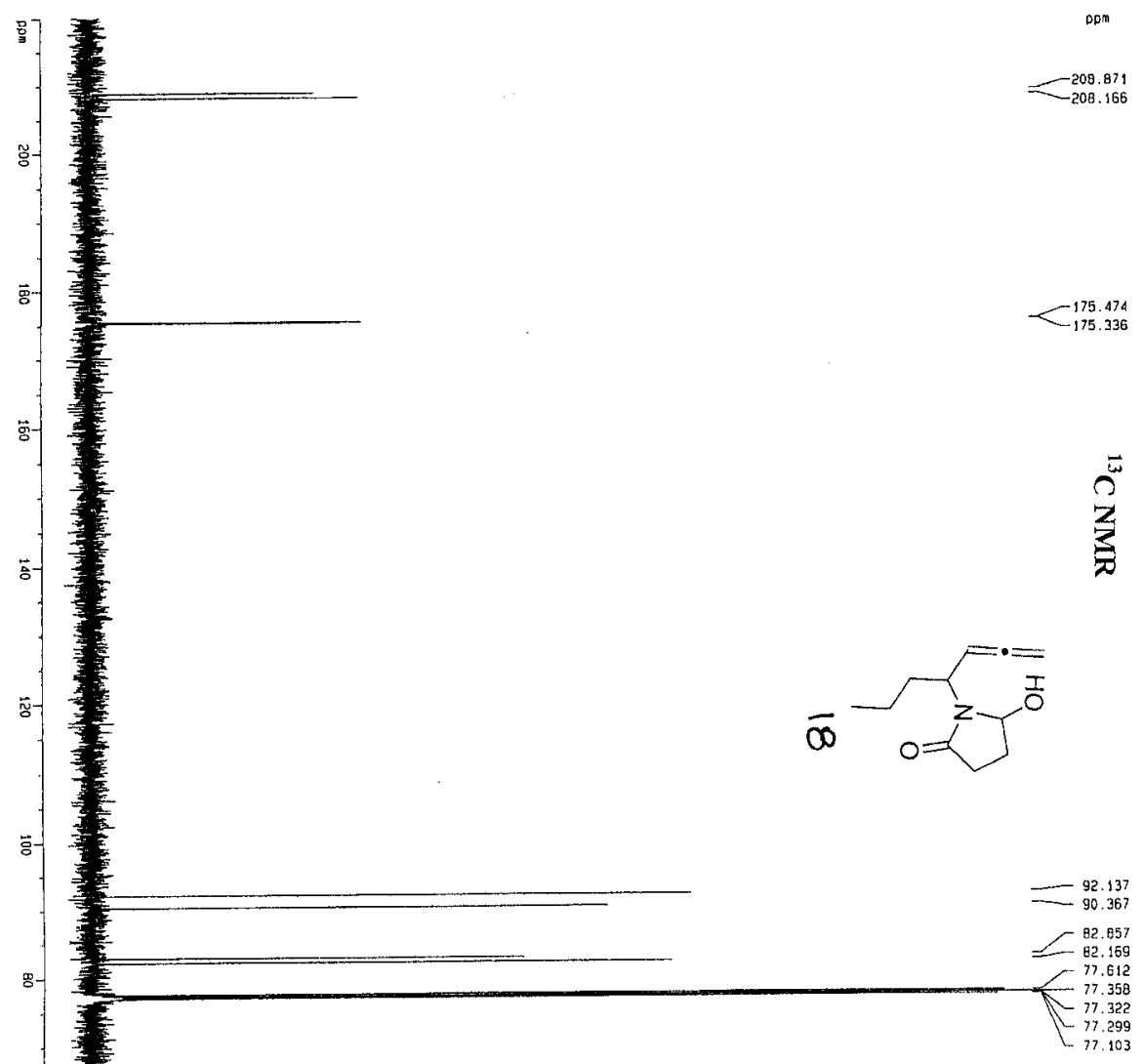

g-

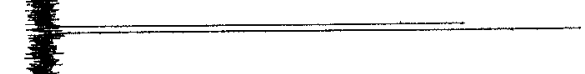

51.167
-50.266

$-$

$-36.371$ 33.787 29.411
29.181

$\begin{array}{r}29.181 \\ -28.861 \\ \hline\end{array}$

s-

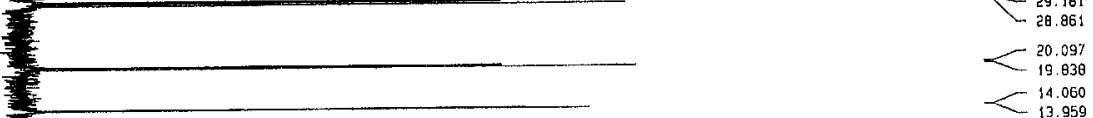

$\circ-$

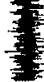

S-

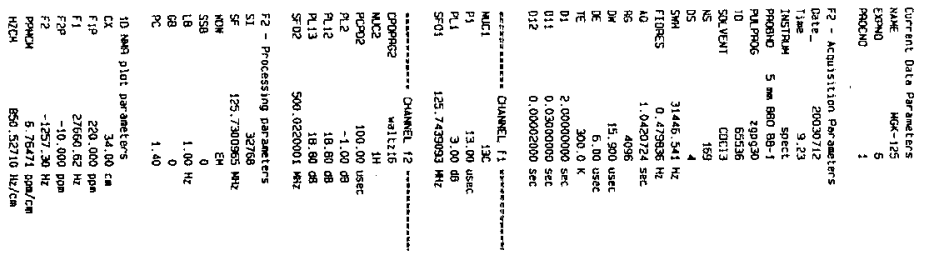




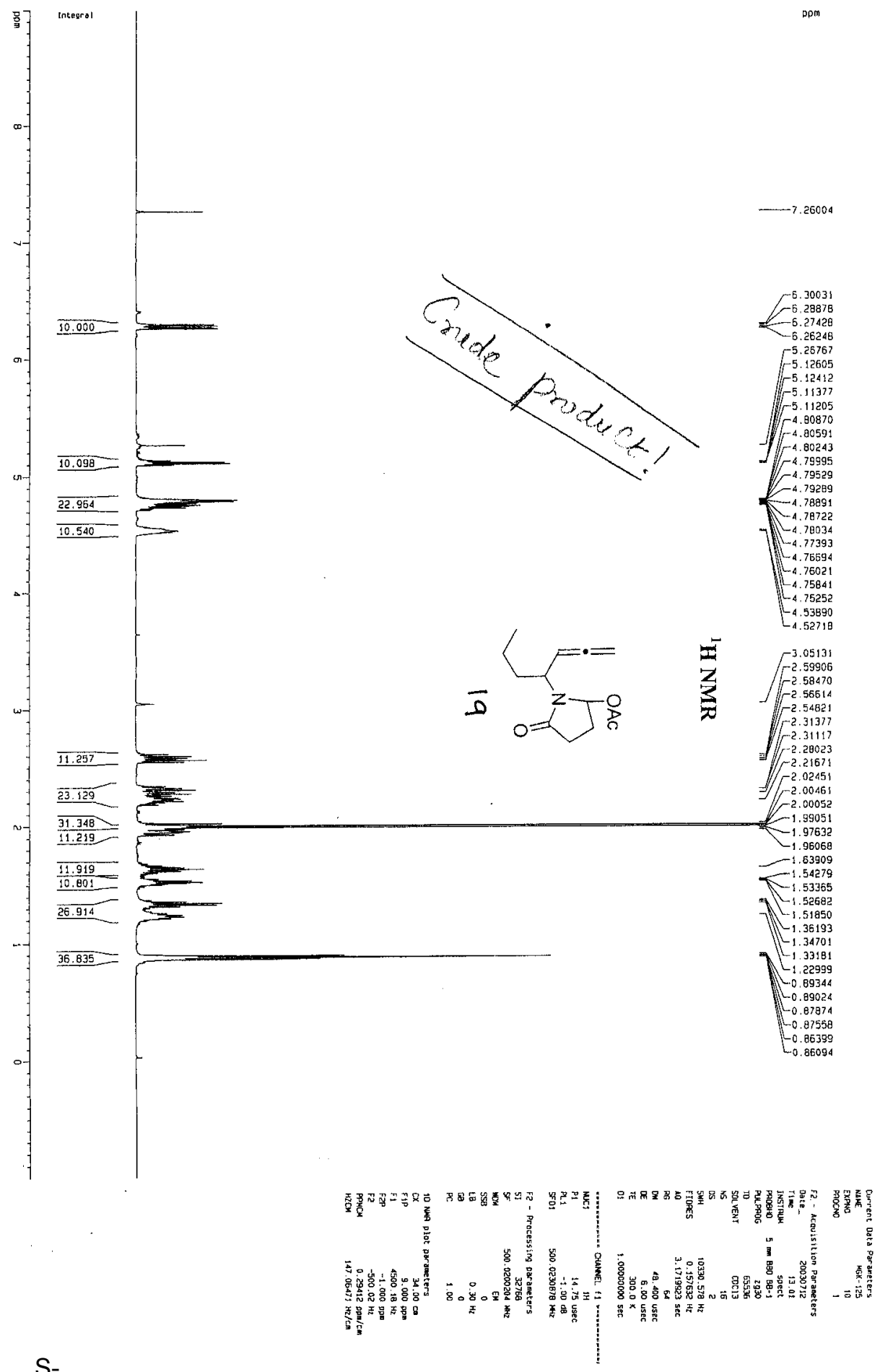

S- 

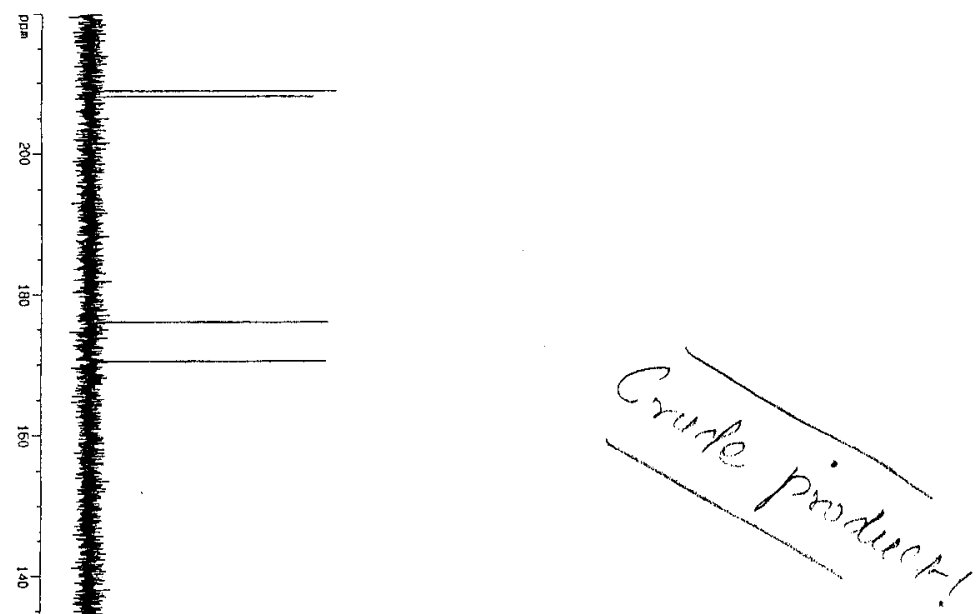

$<-176.075$

$<170.472$
$-\quad 170.439$

$-170.439$

$\overline{\mathrm{Q}}-$
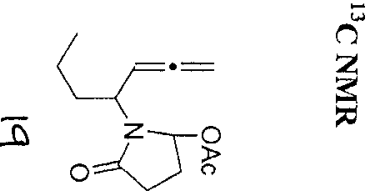

형

g-

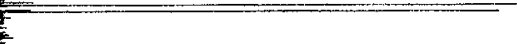
49.722

- 35.632 33. 158 28.934 27.304 27.130 21.586 21.519

19.825

$-19.688$

14.054

13.054
-13.892

S-

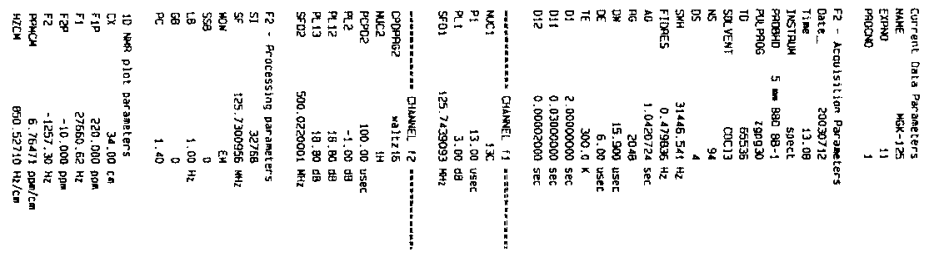


$\downarrow \varepsilon-S$

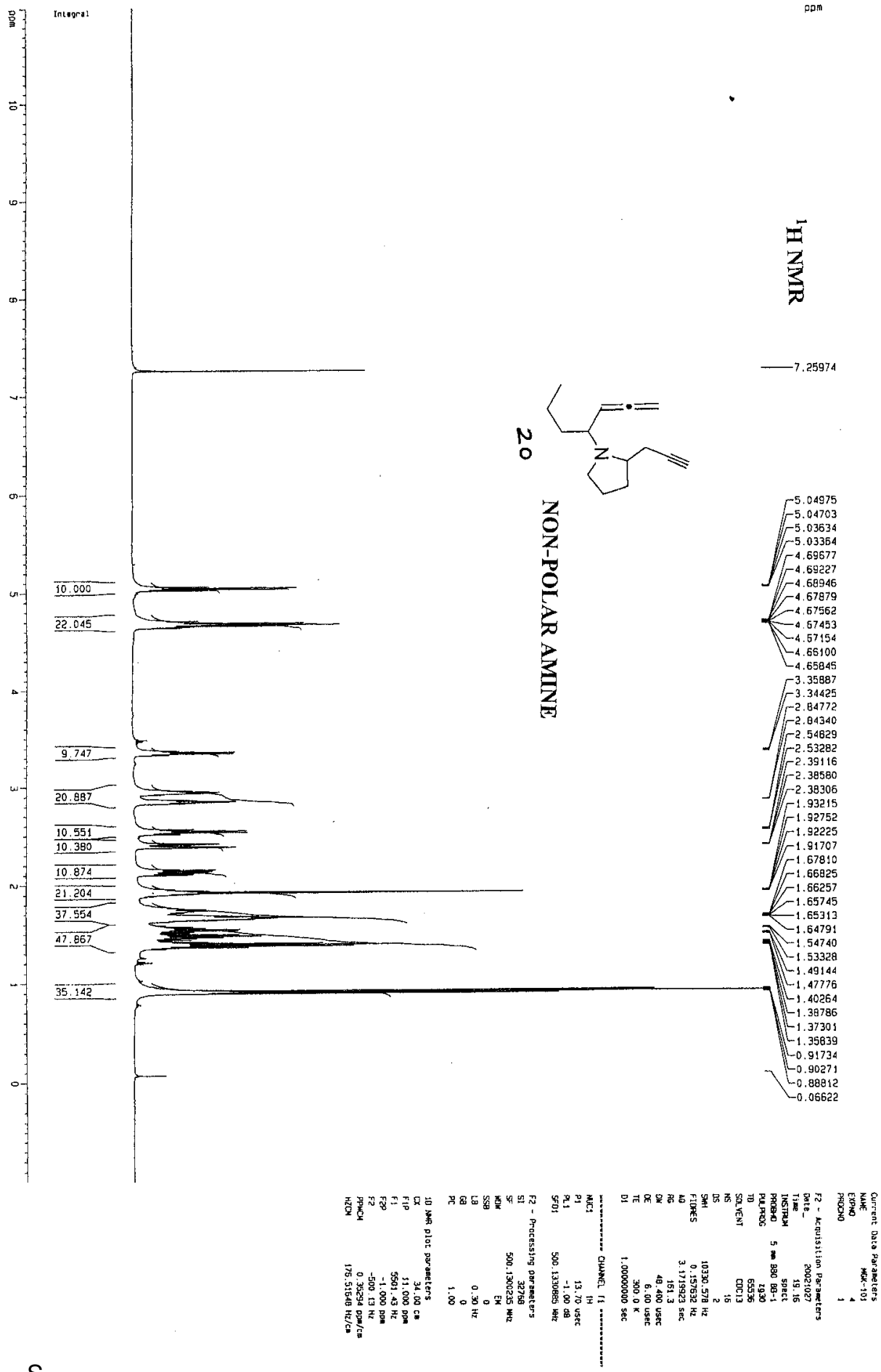

S- 

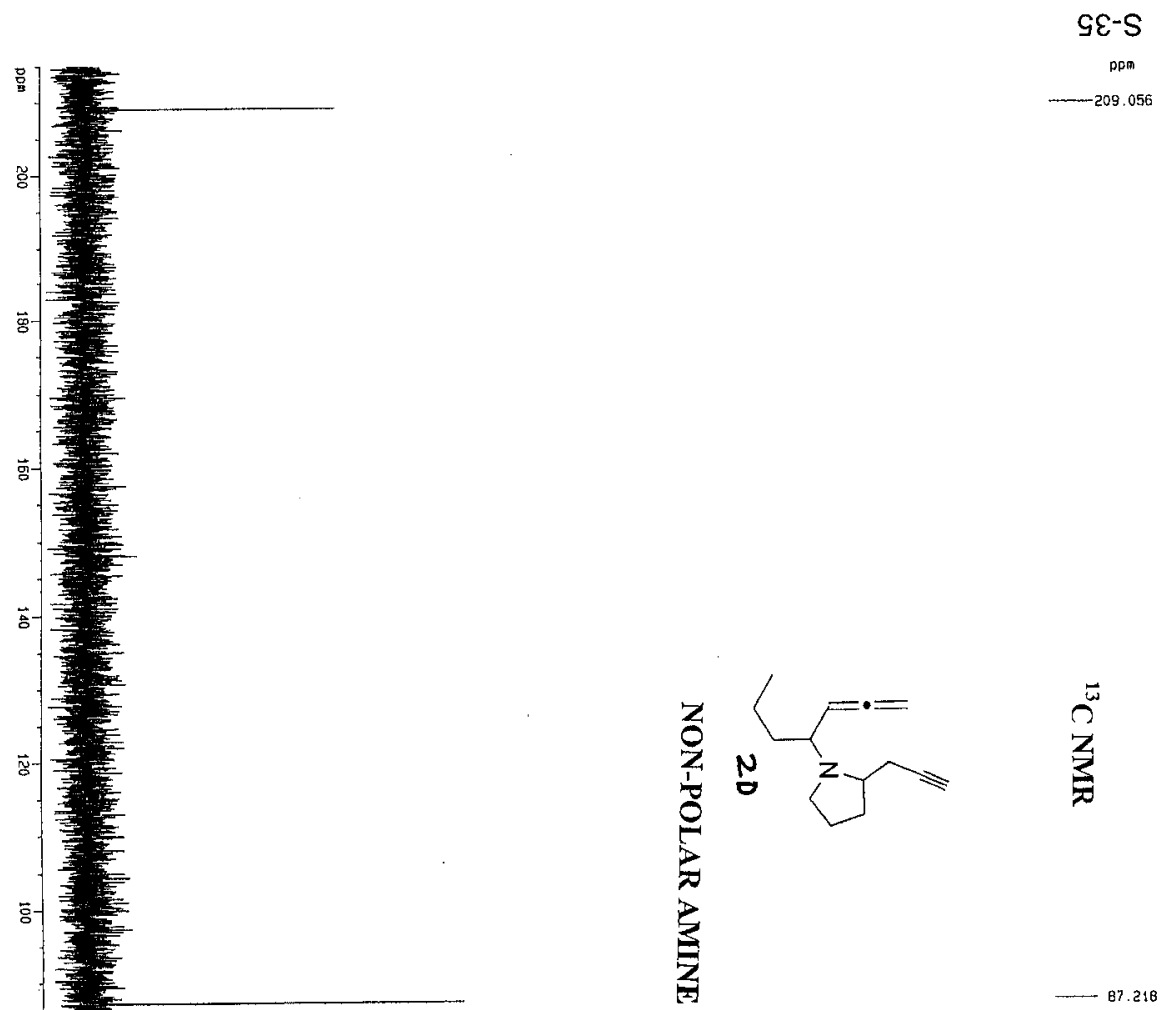

\%

妻 83.234 77.621 77.367
77.114 74.581

- 69.156

s. 46.399

b-
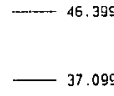

- 30.887

- 24.34 22.975 20.211

$\approx$

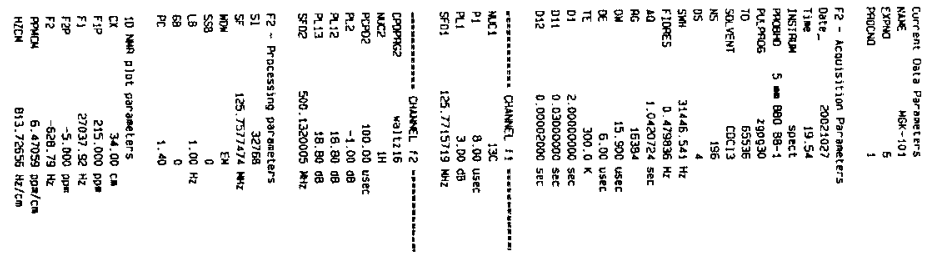

S- 


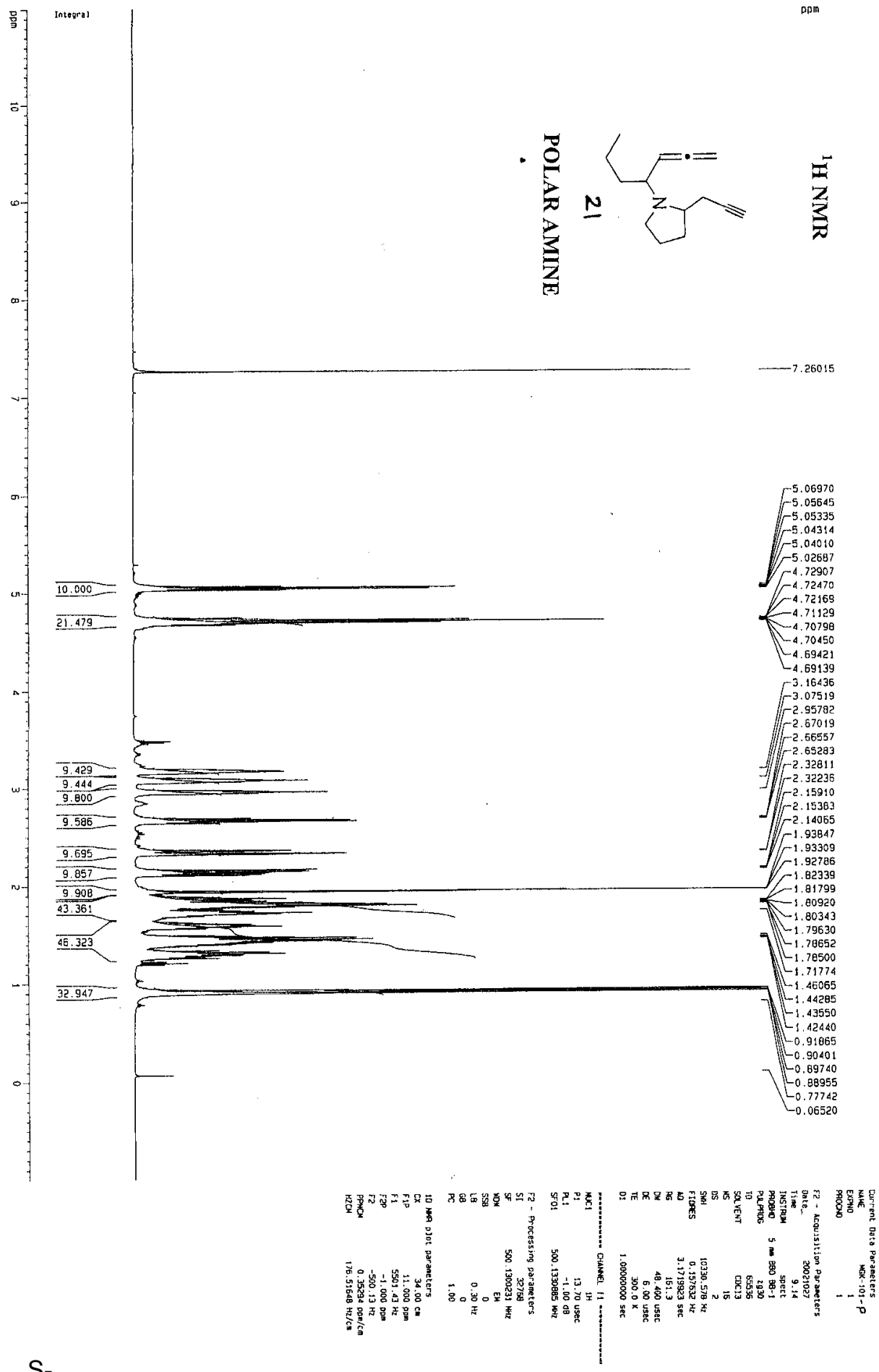




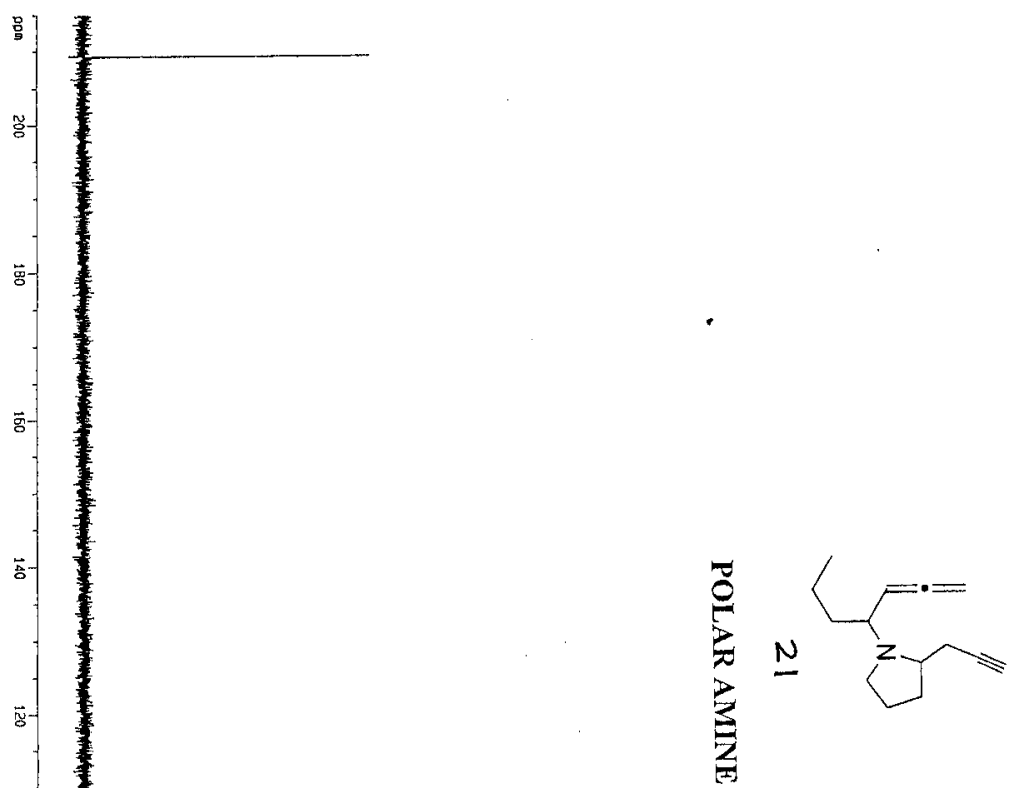

$\overbrace{}^{2}$

s-

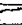
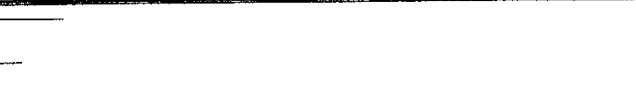

ב_+

61.687
$-\quad 58.410$

$-51.995$

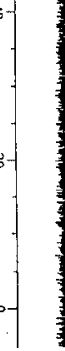

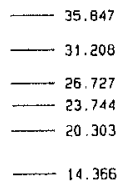

S-

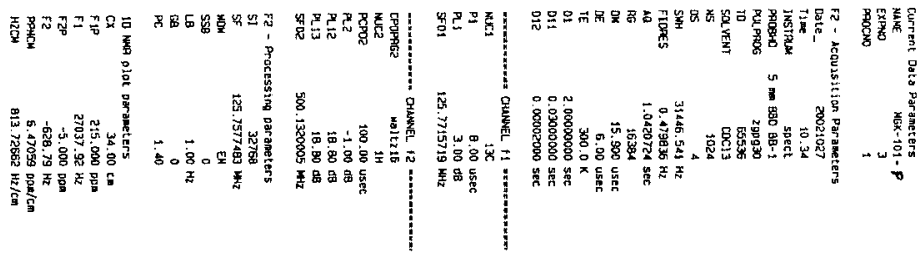




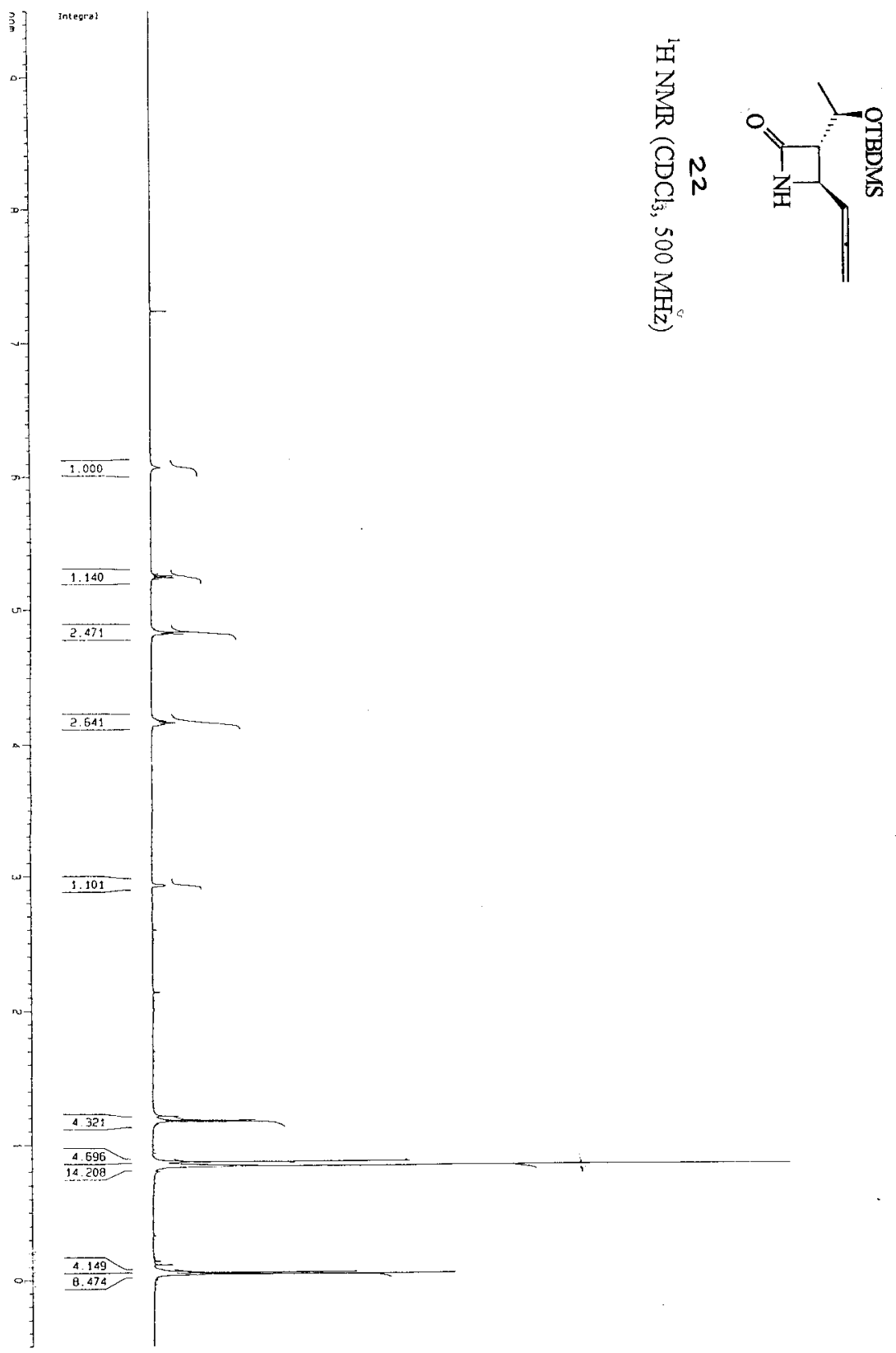

S- 


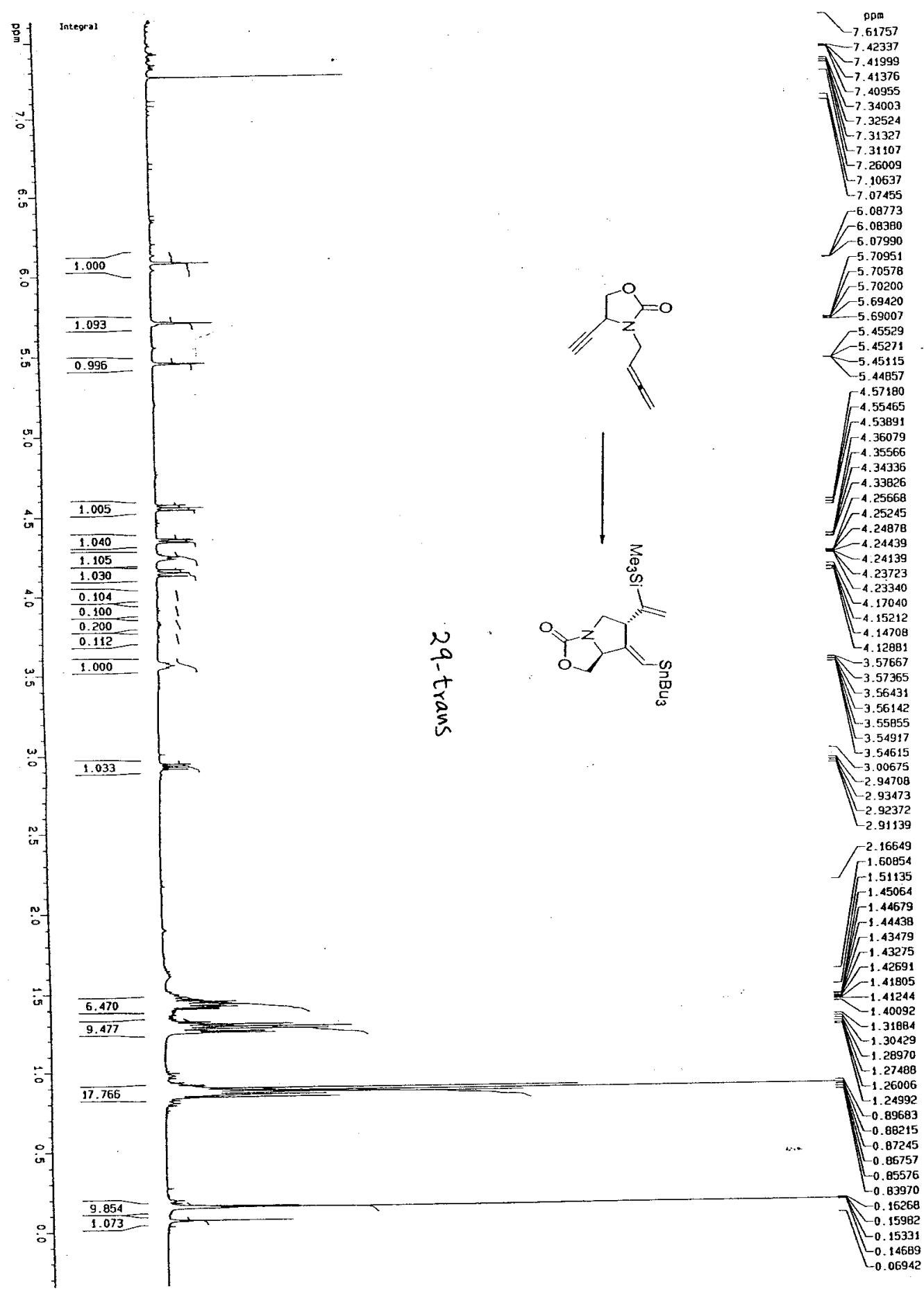




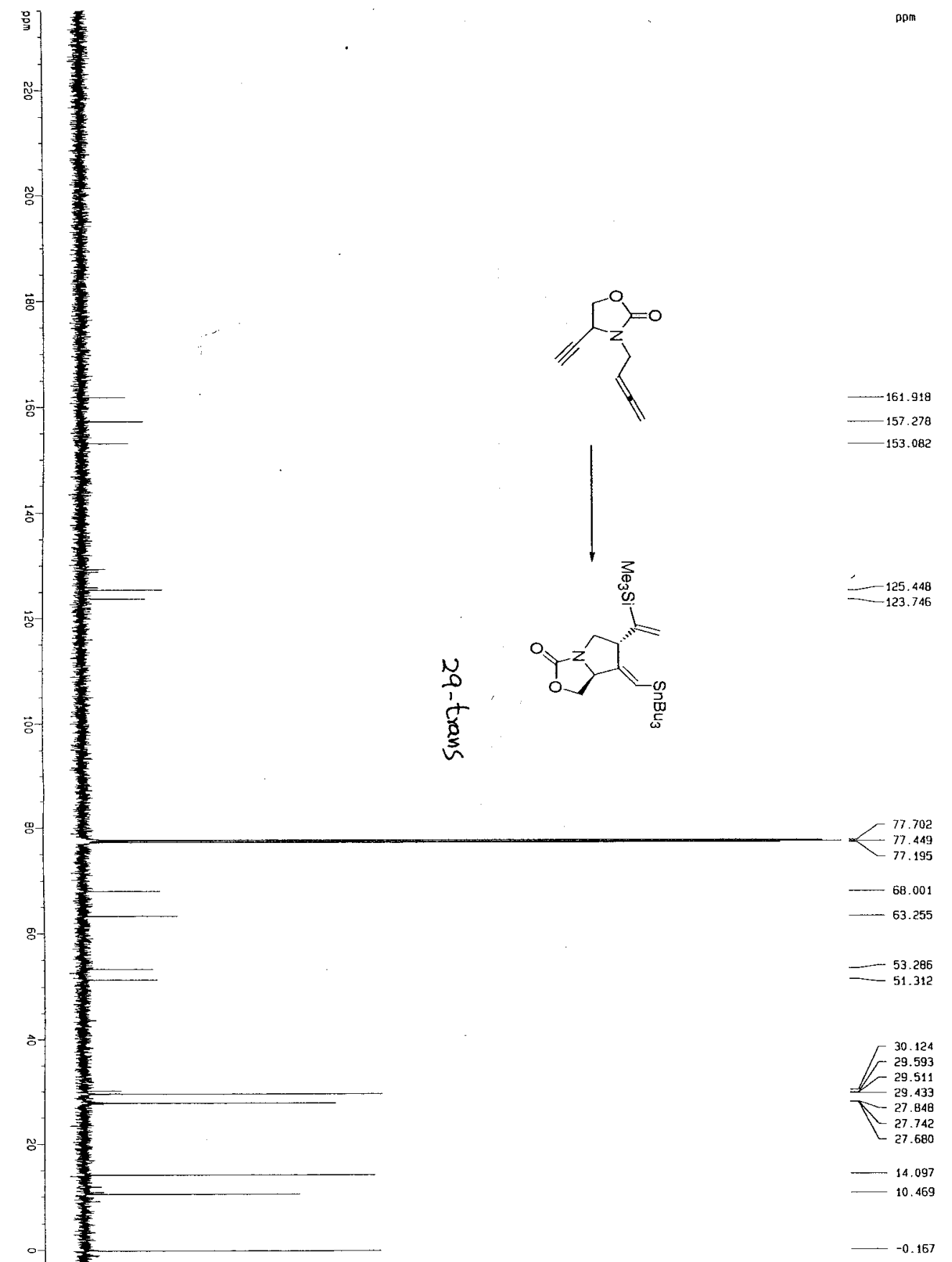




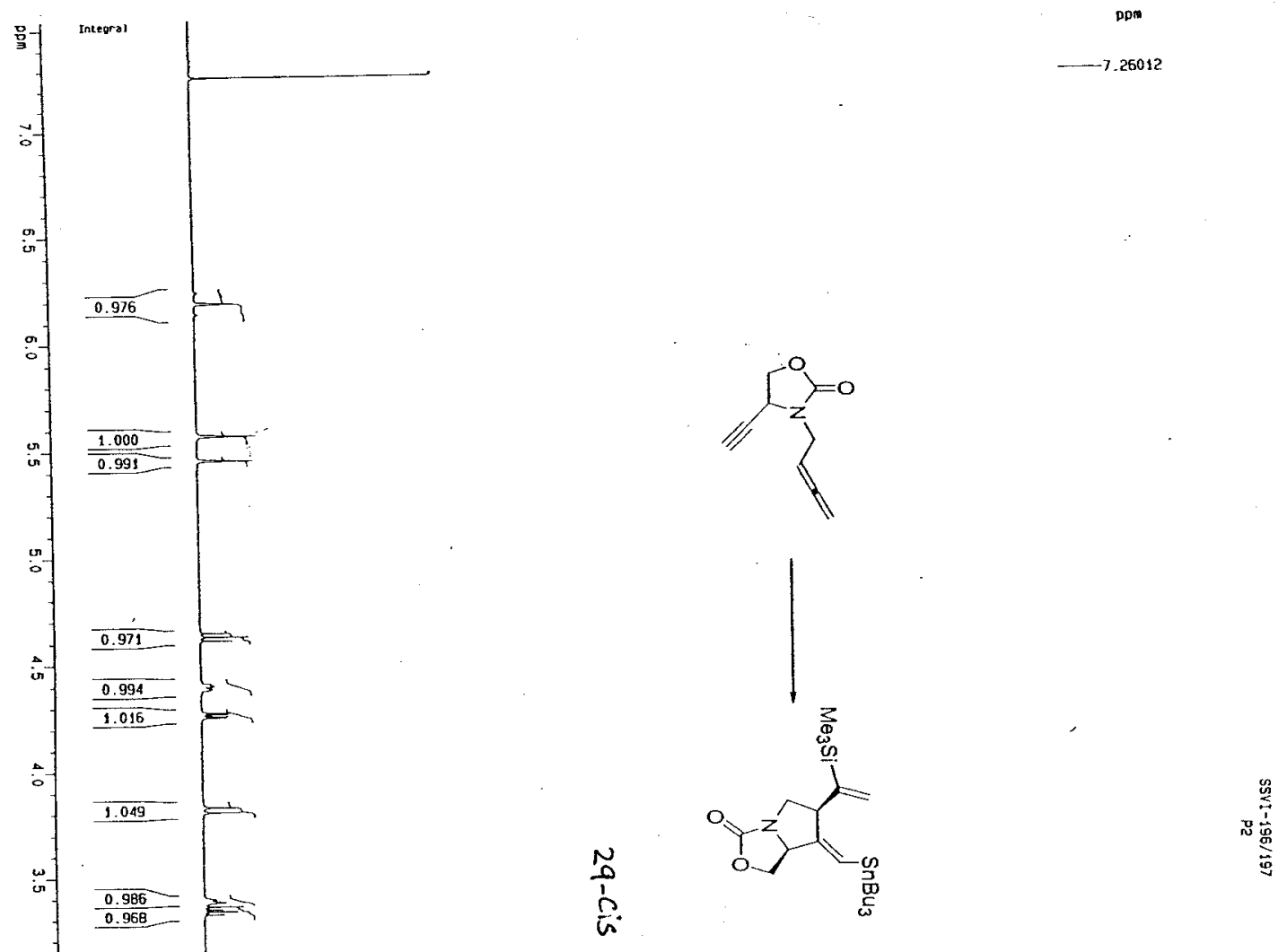

in

0

o

1

$\int_{0}^{\frac{5.263}{11.174}}$

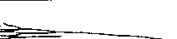

$E$

D 


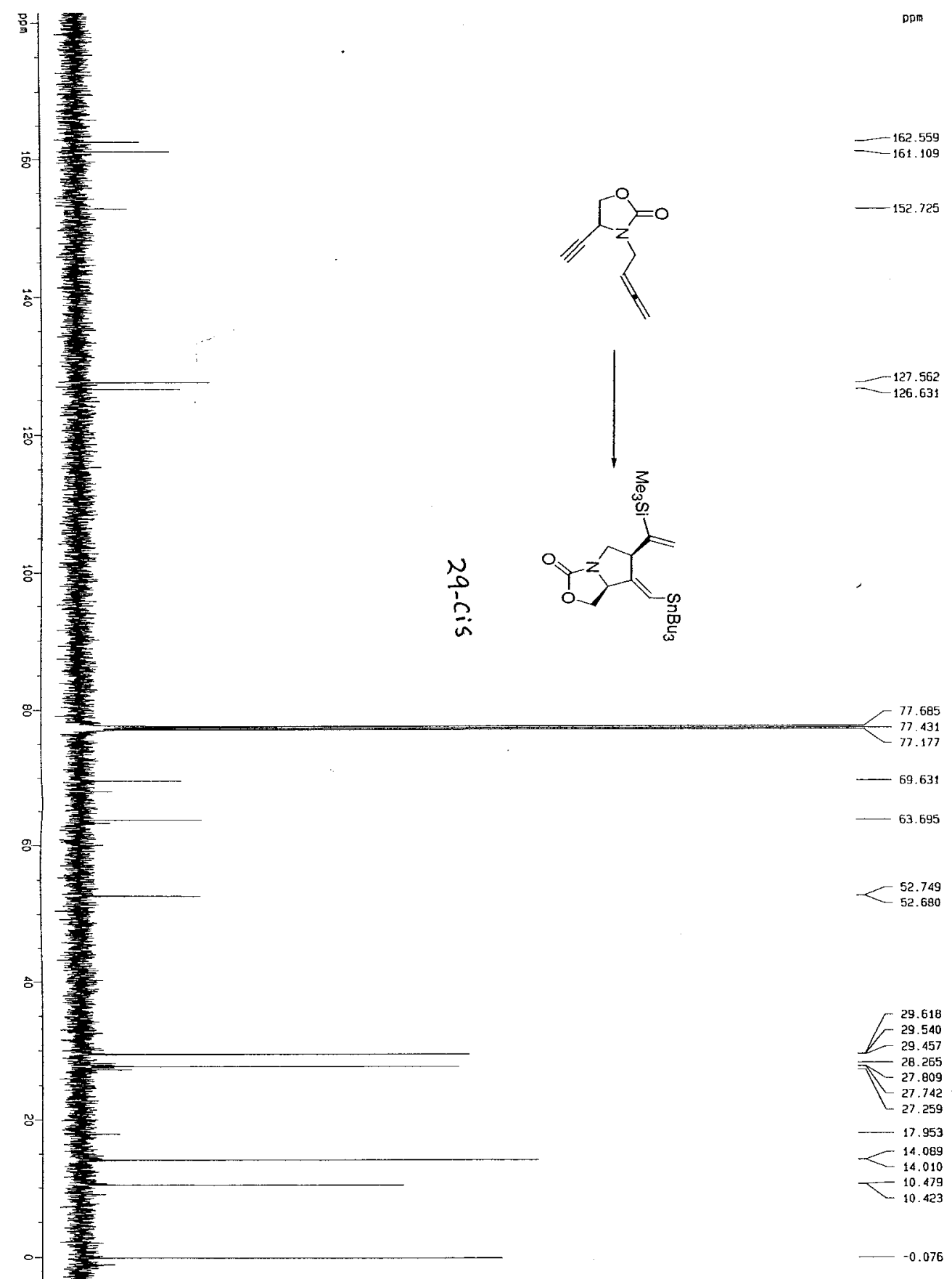




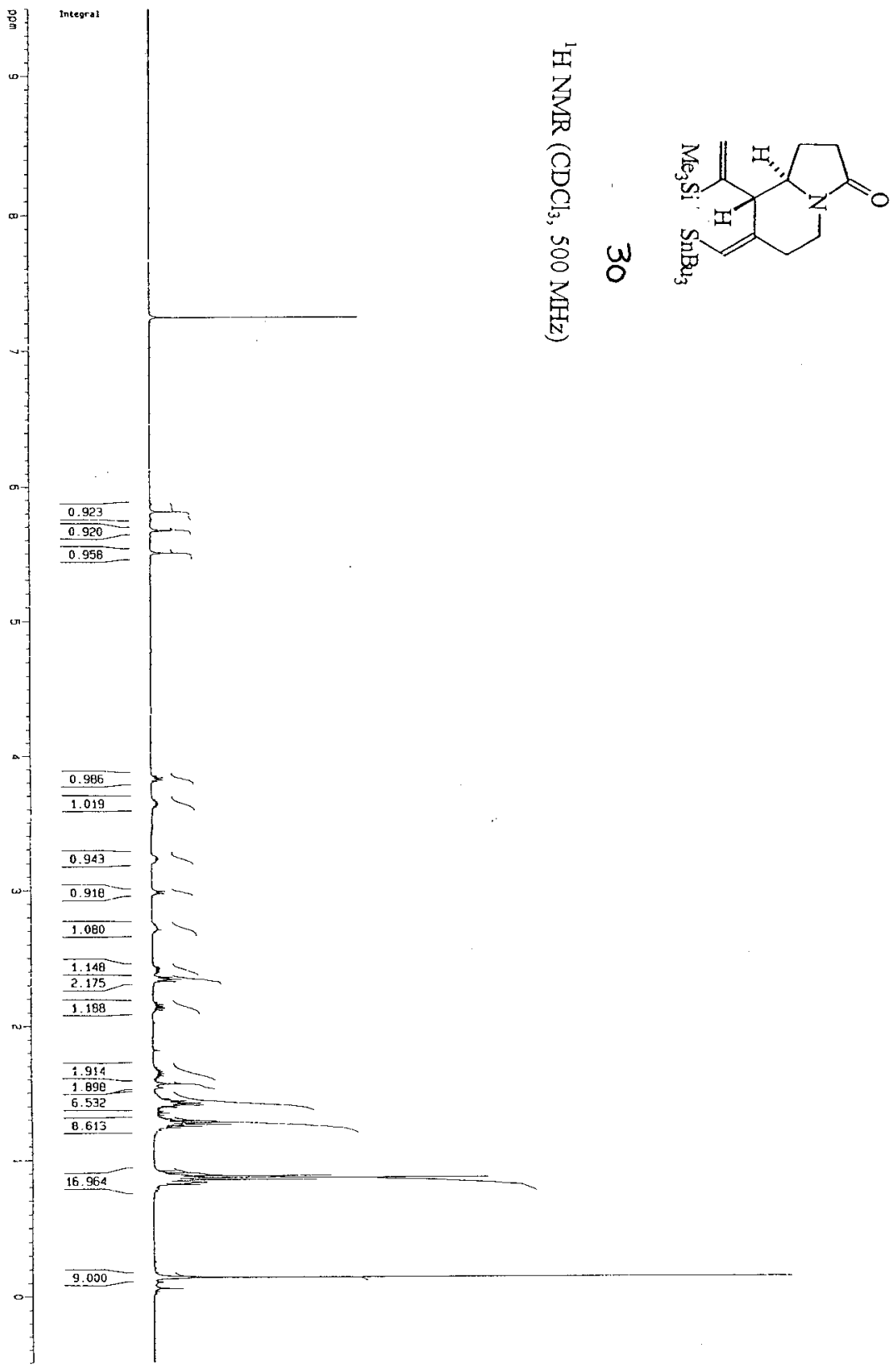

S- 


$$
t^{f}
$$




$$
H^{* x}
$$




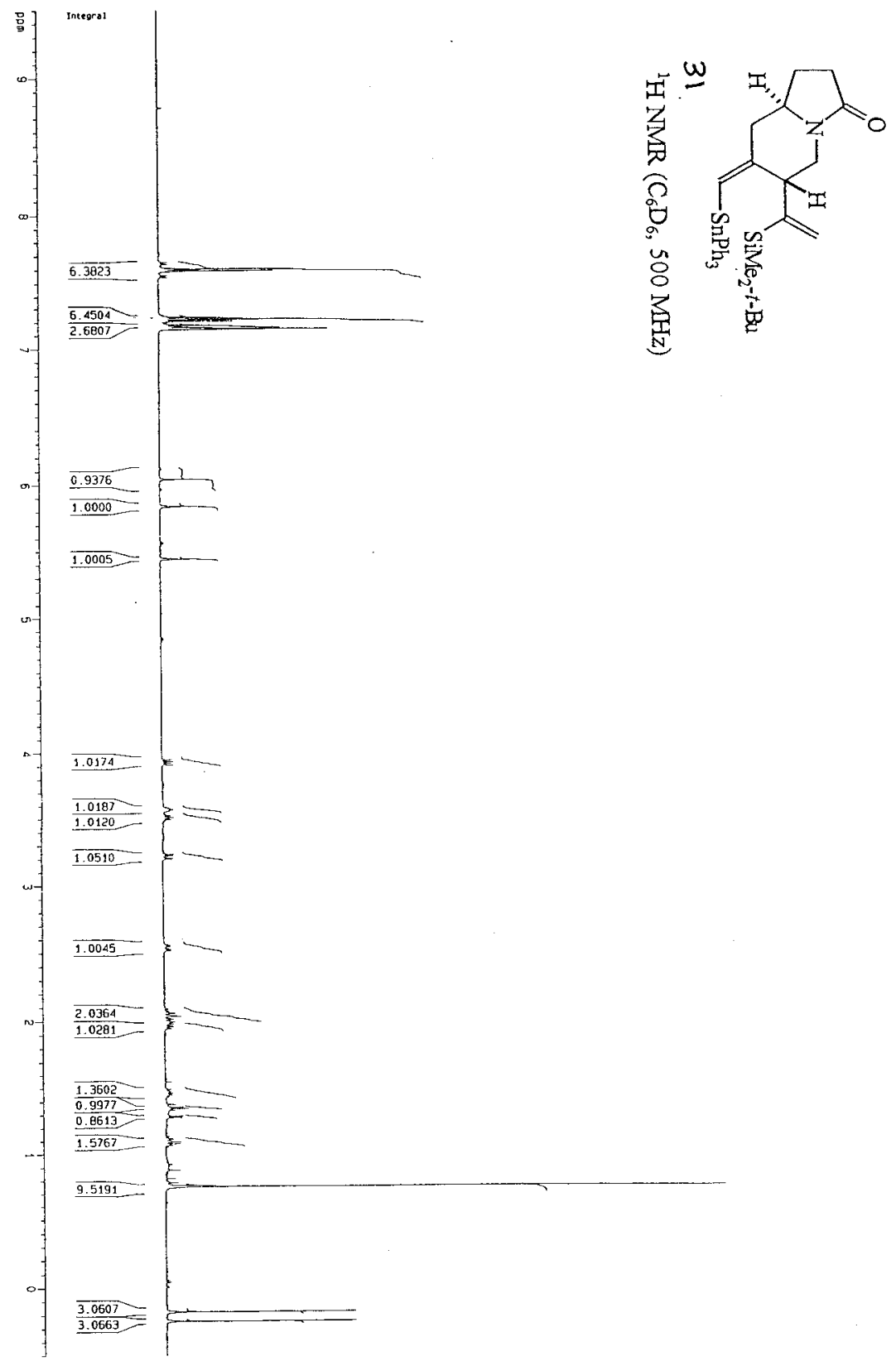

S- 


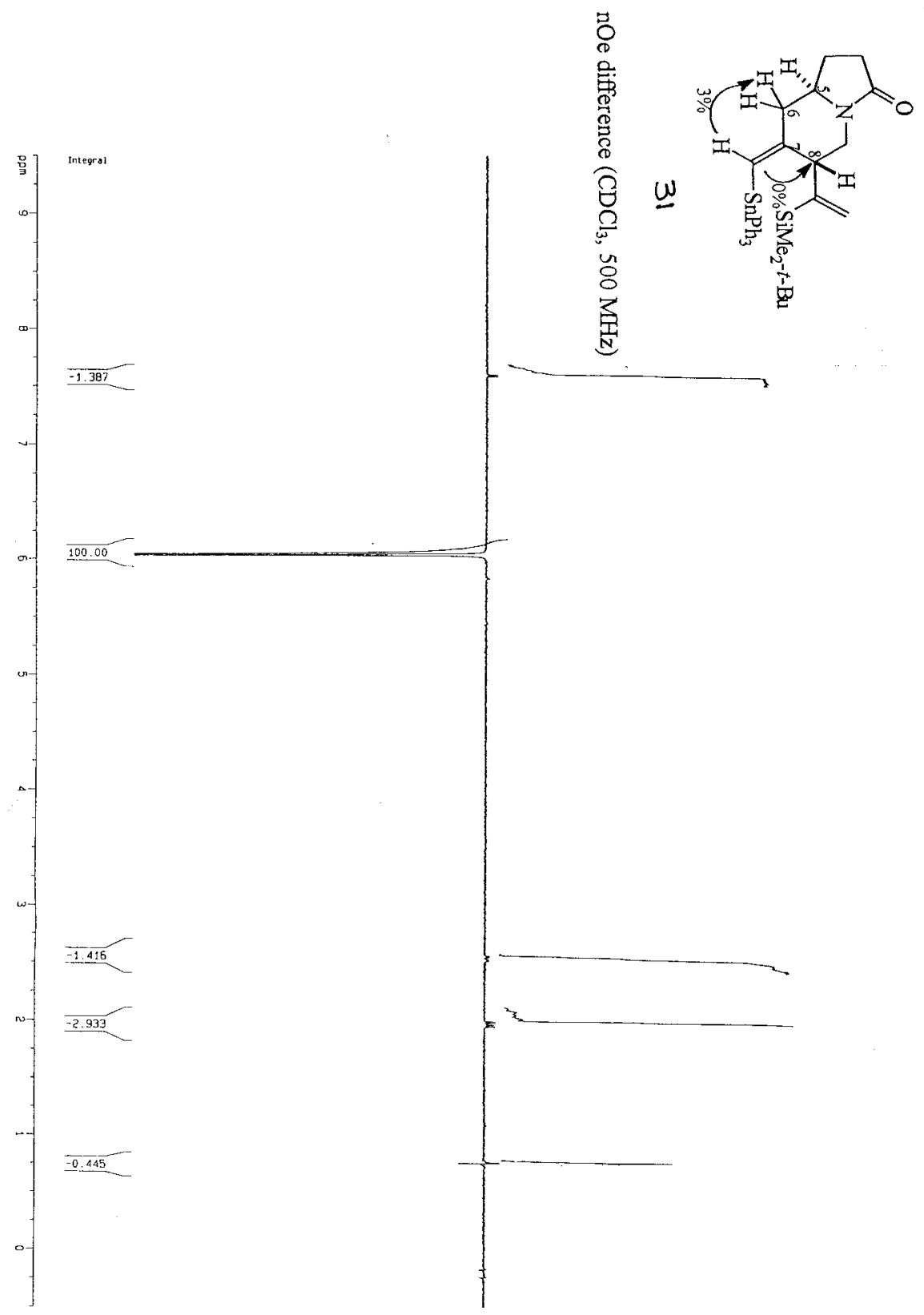

S-

58 


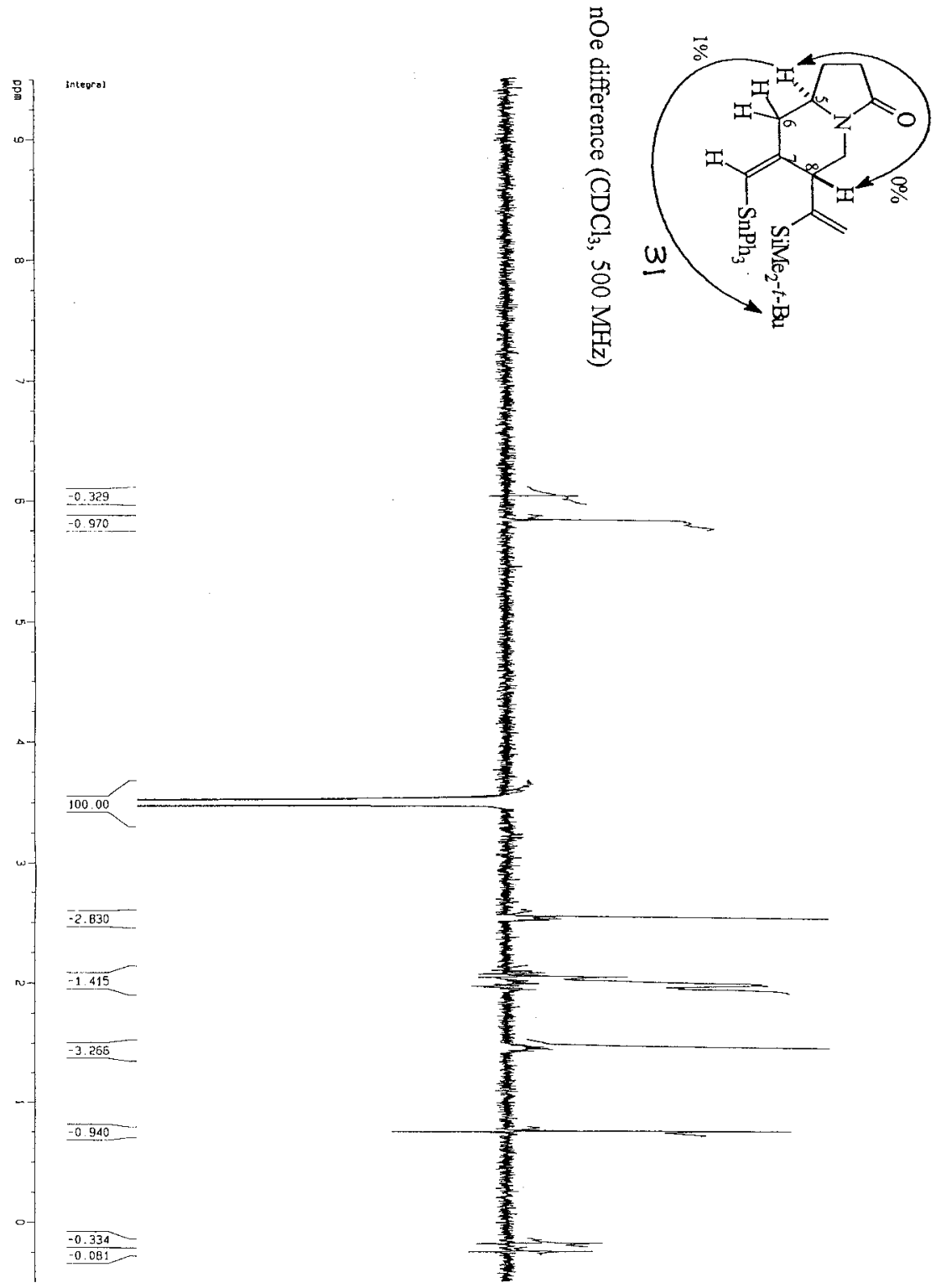

S-

59 


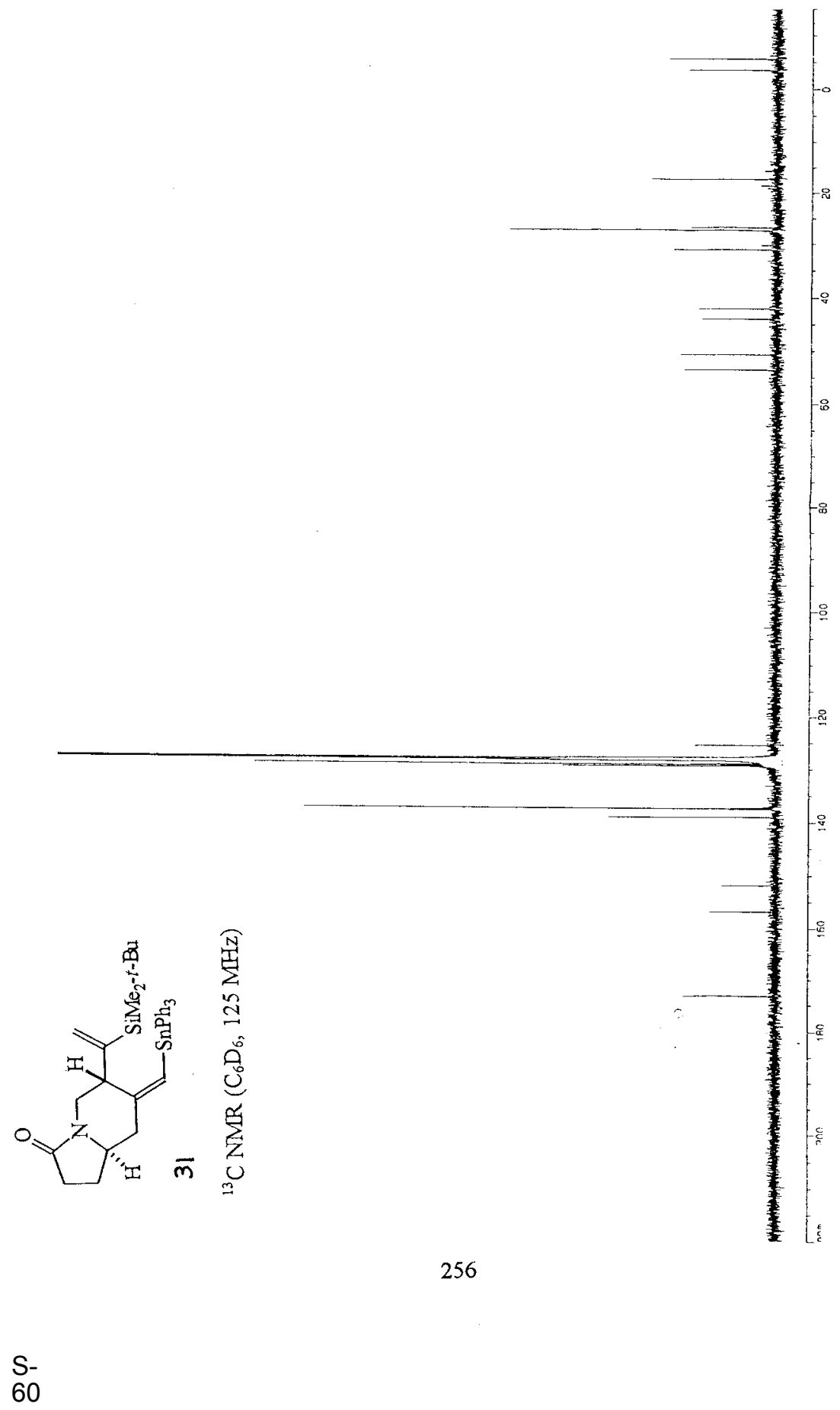



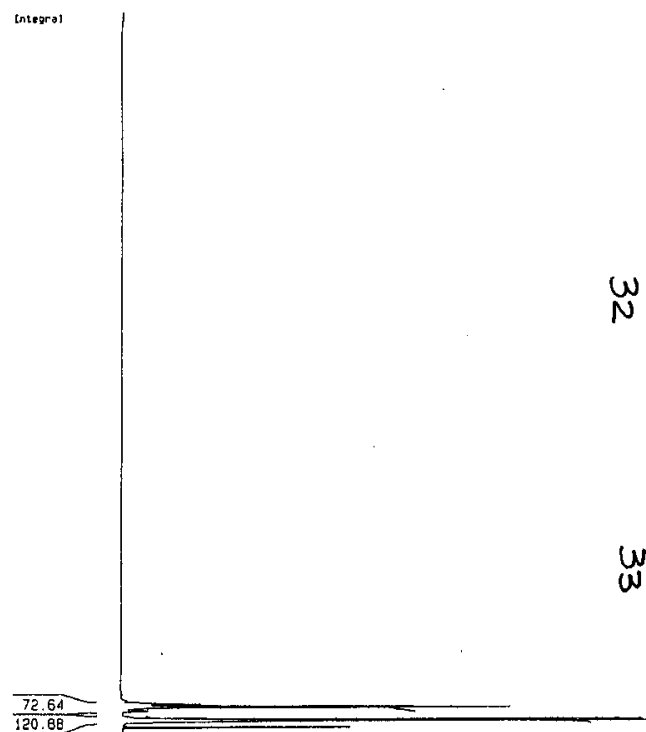

$\omega$
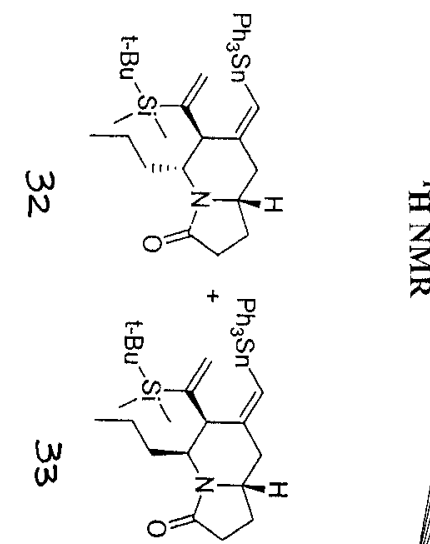

$\frac{3}{3}$
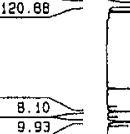

9.93

9.77

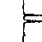

9.66

$E$

$9.40=$

17.73

$\frac{12.41}{11.62}=$

$\frac{1.62}{10.06}$

$\frac{12.54}{10.54}$

20.21

$34.78 \mathrm{~S}$

$\frac{20.41}{91.05}$

$\frac{30.33}{30.00}=$
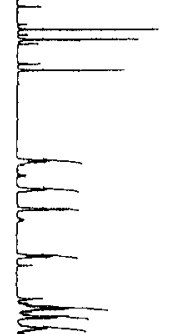

5

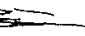

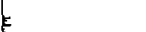

3
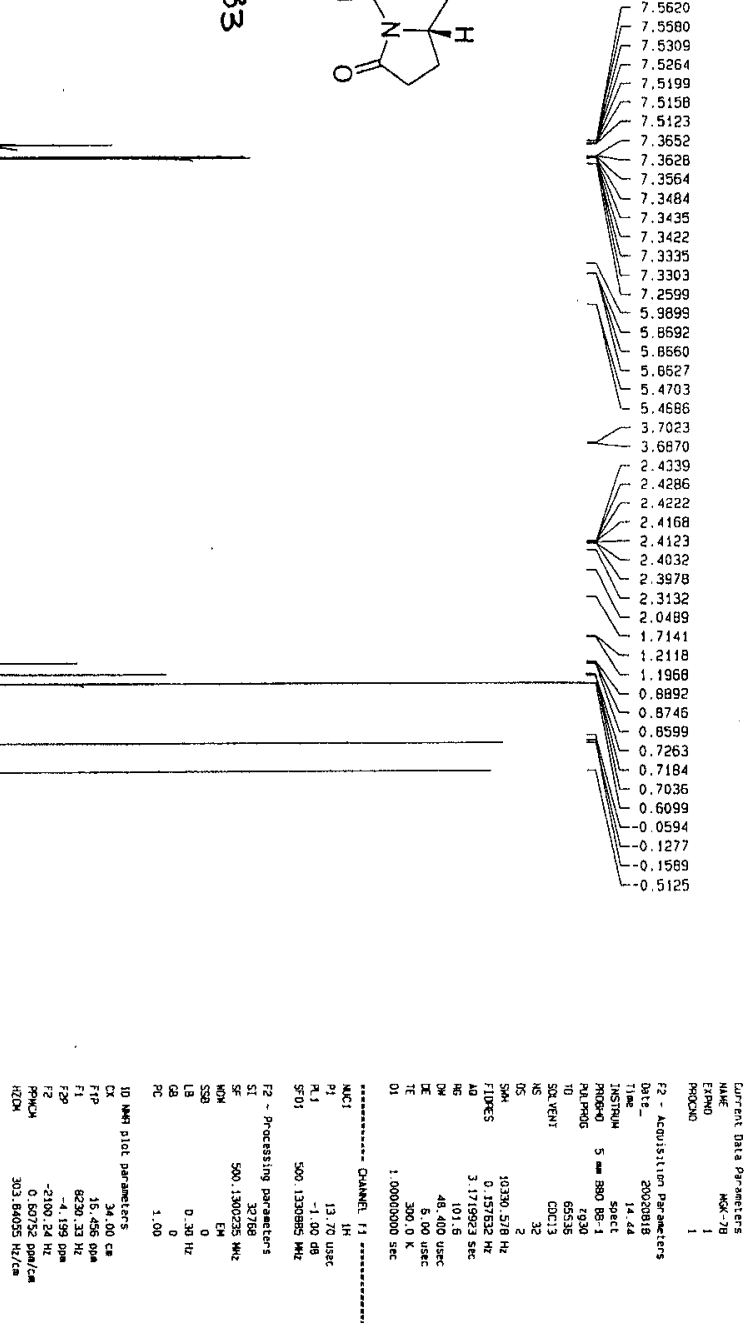

S- 


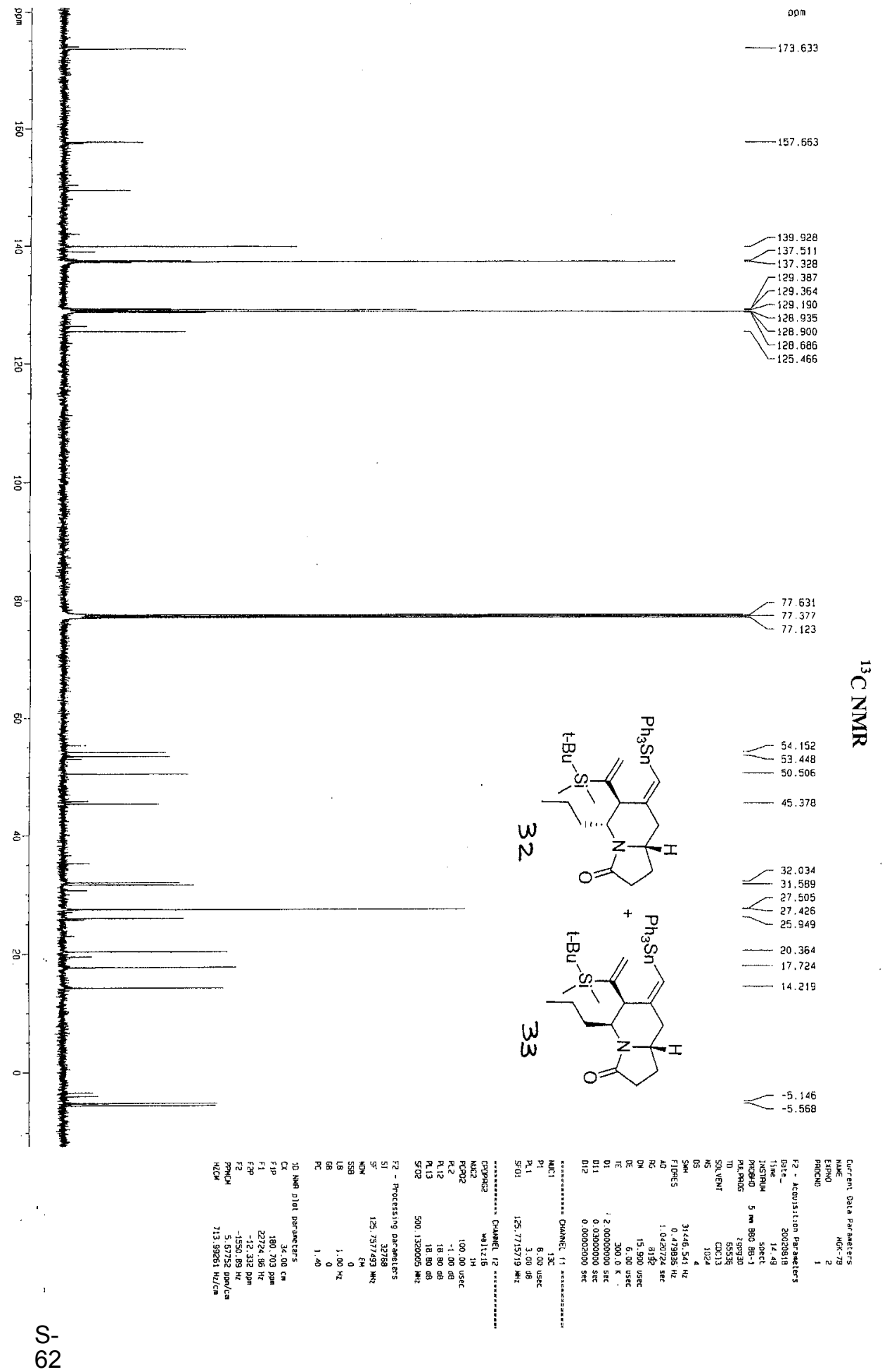




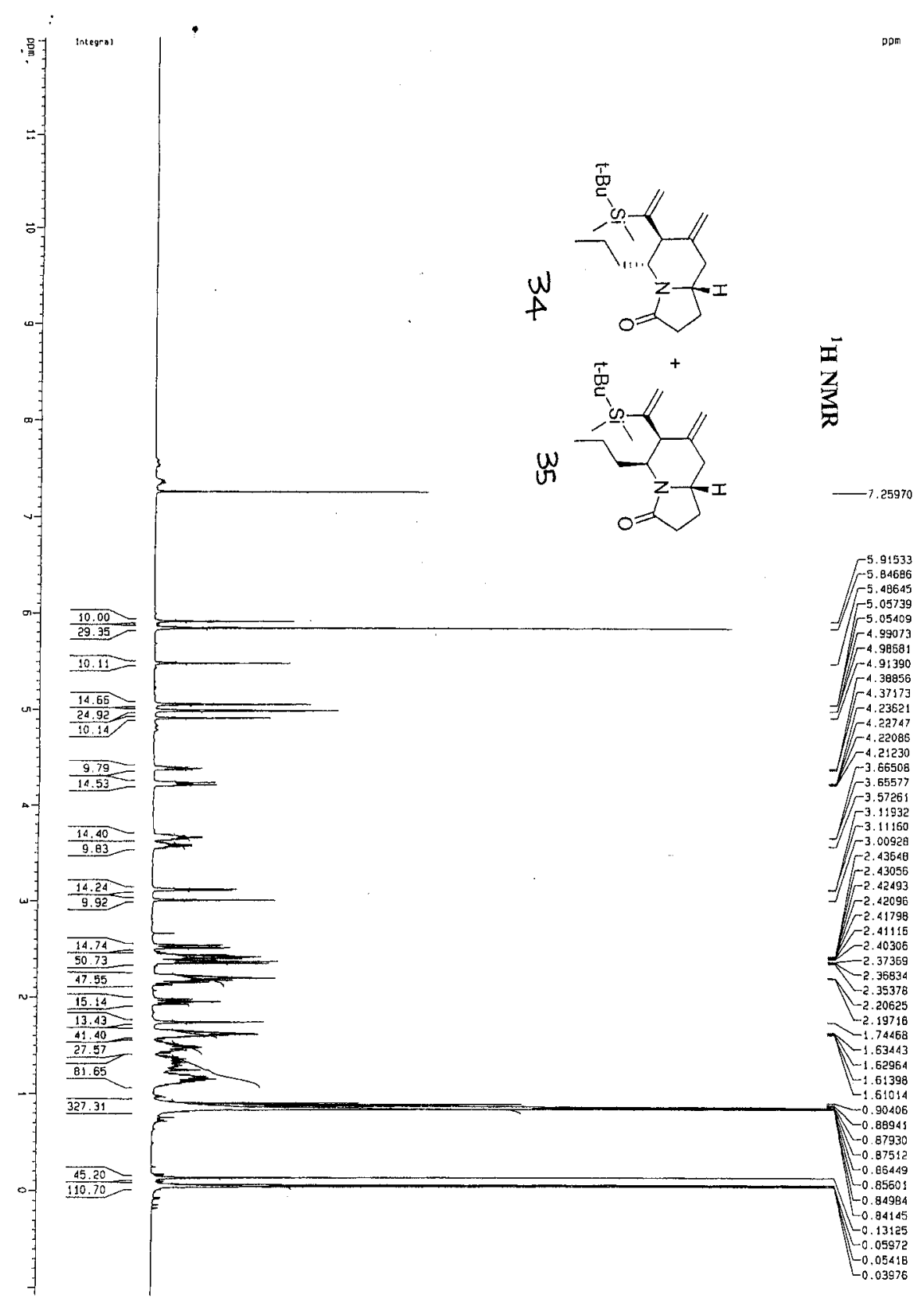

S-

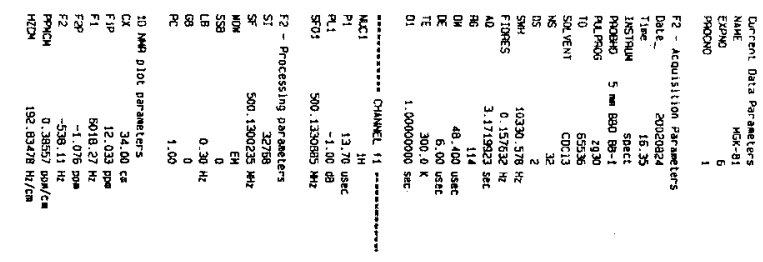




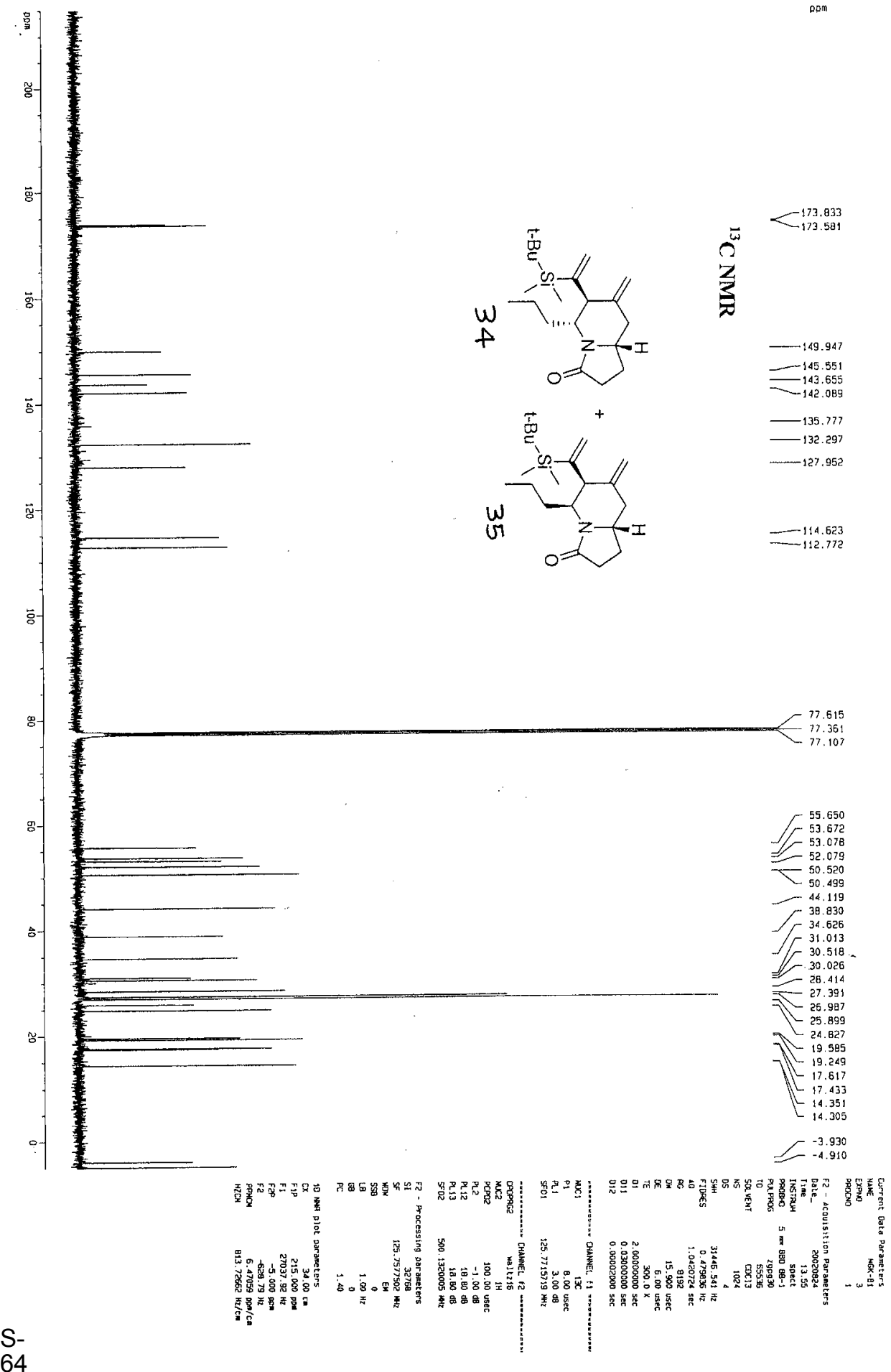




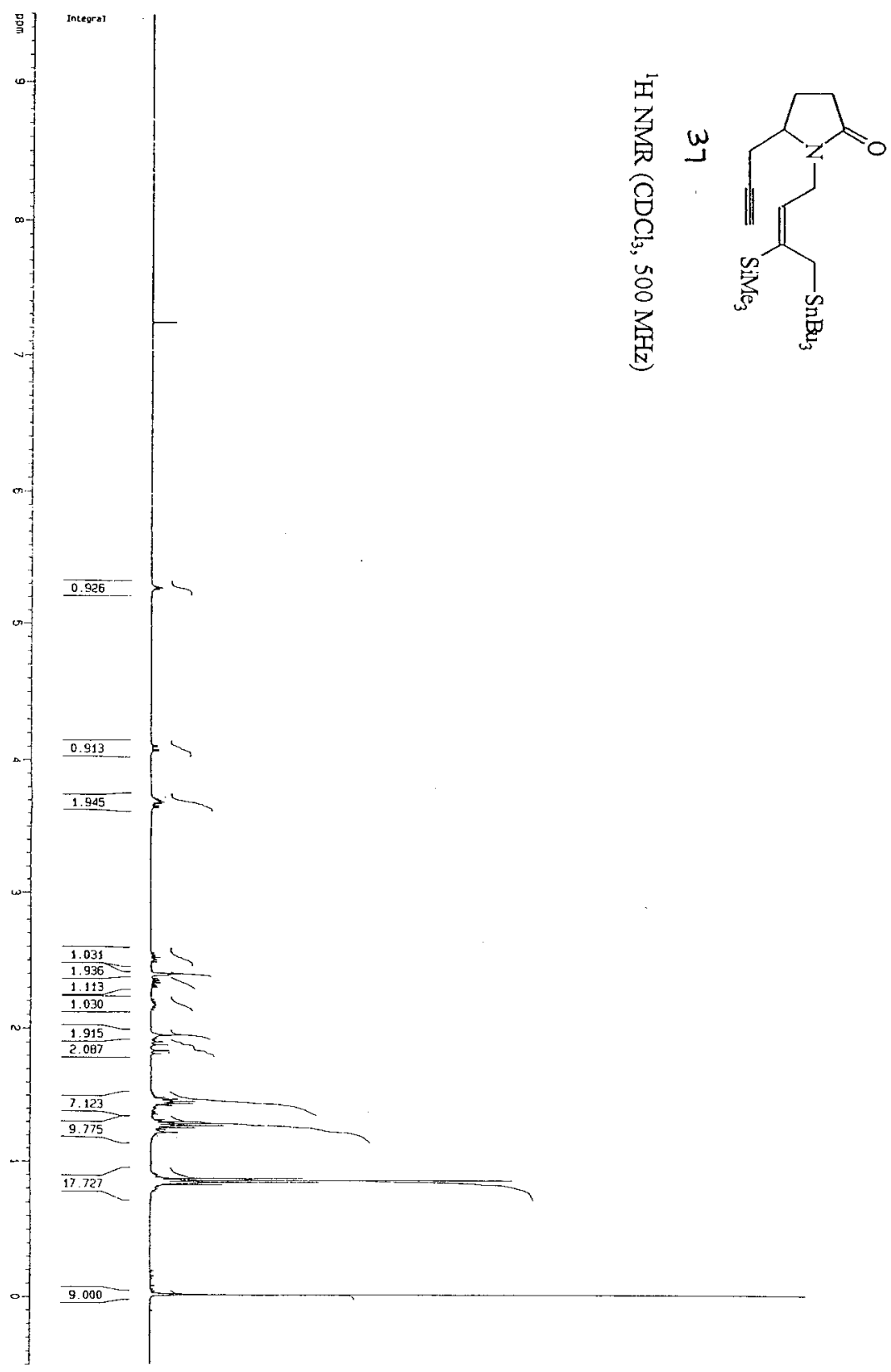

S-

65 


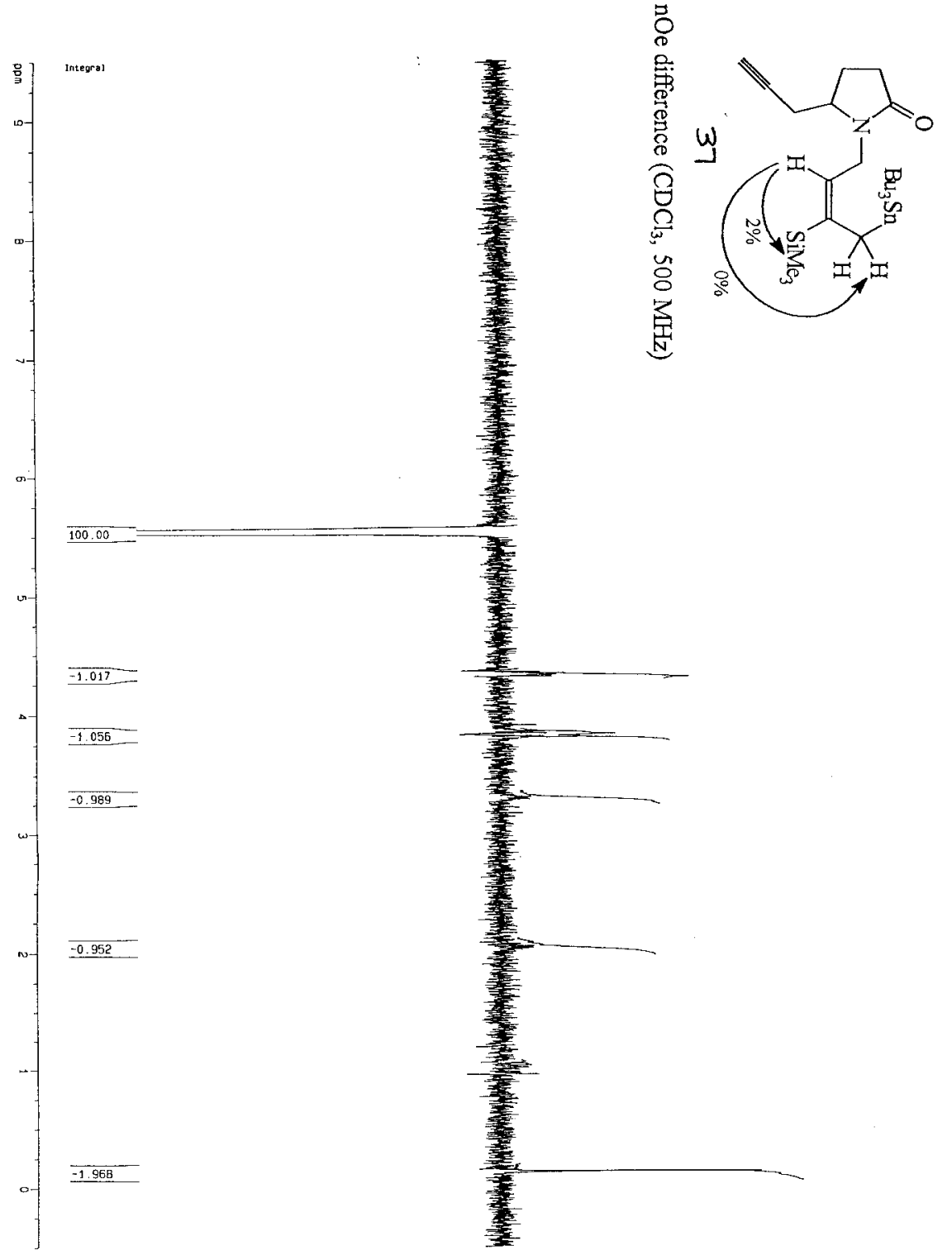

S-

66 


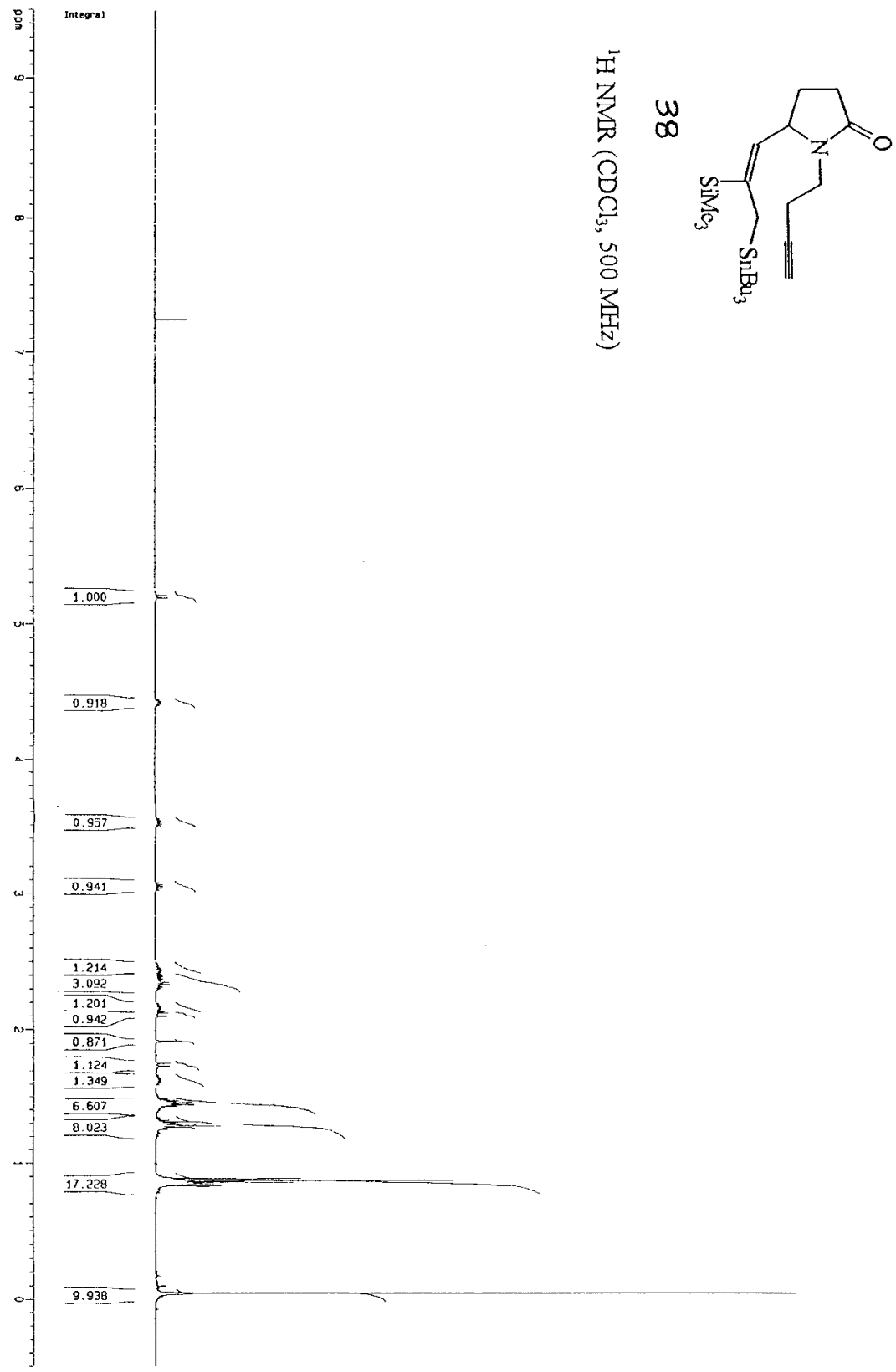

S- 


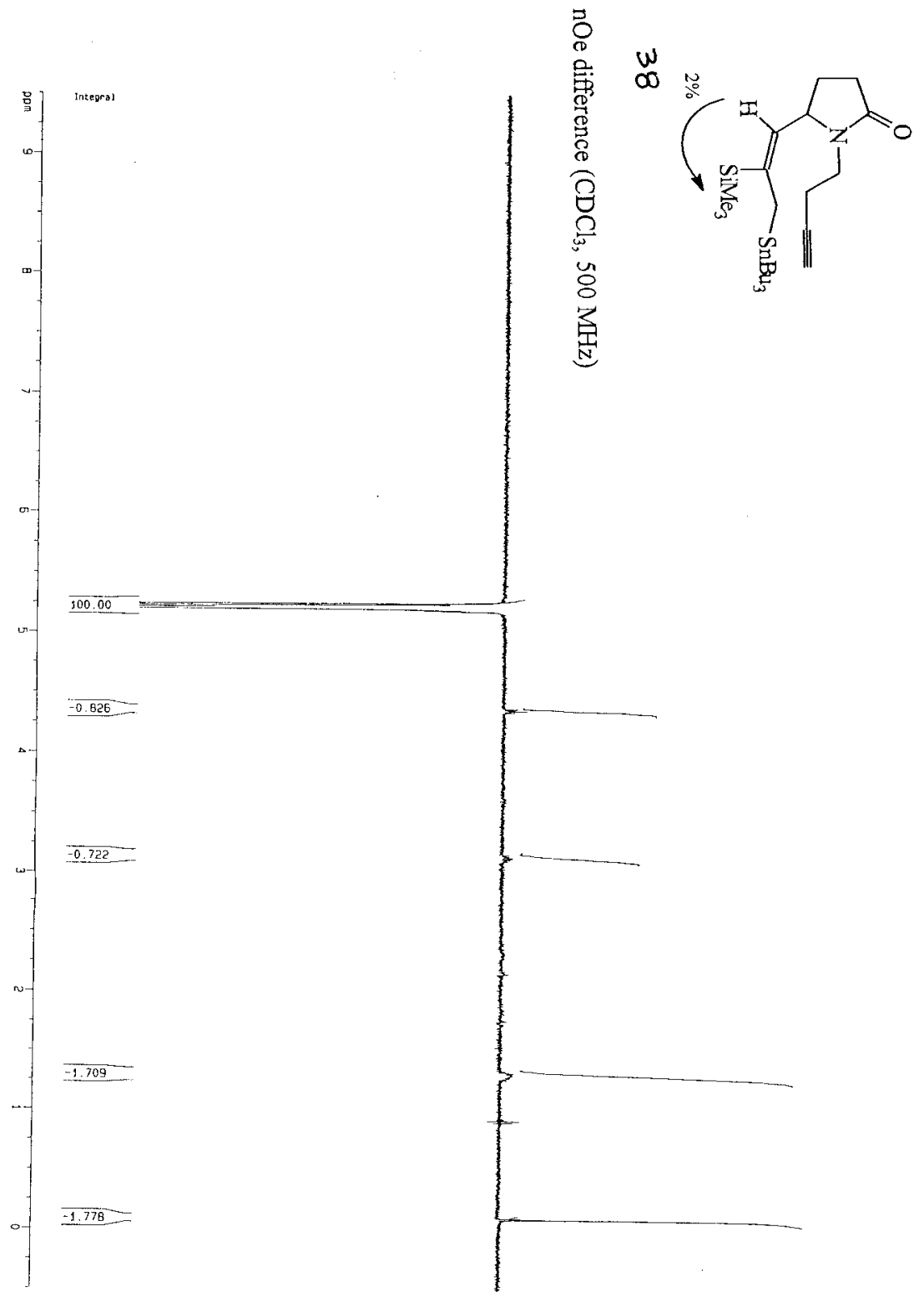

S-

68 


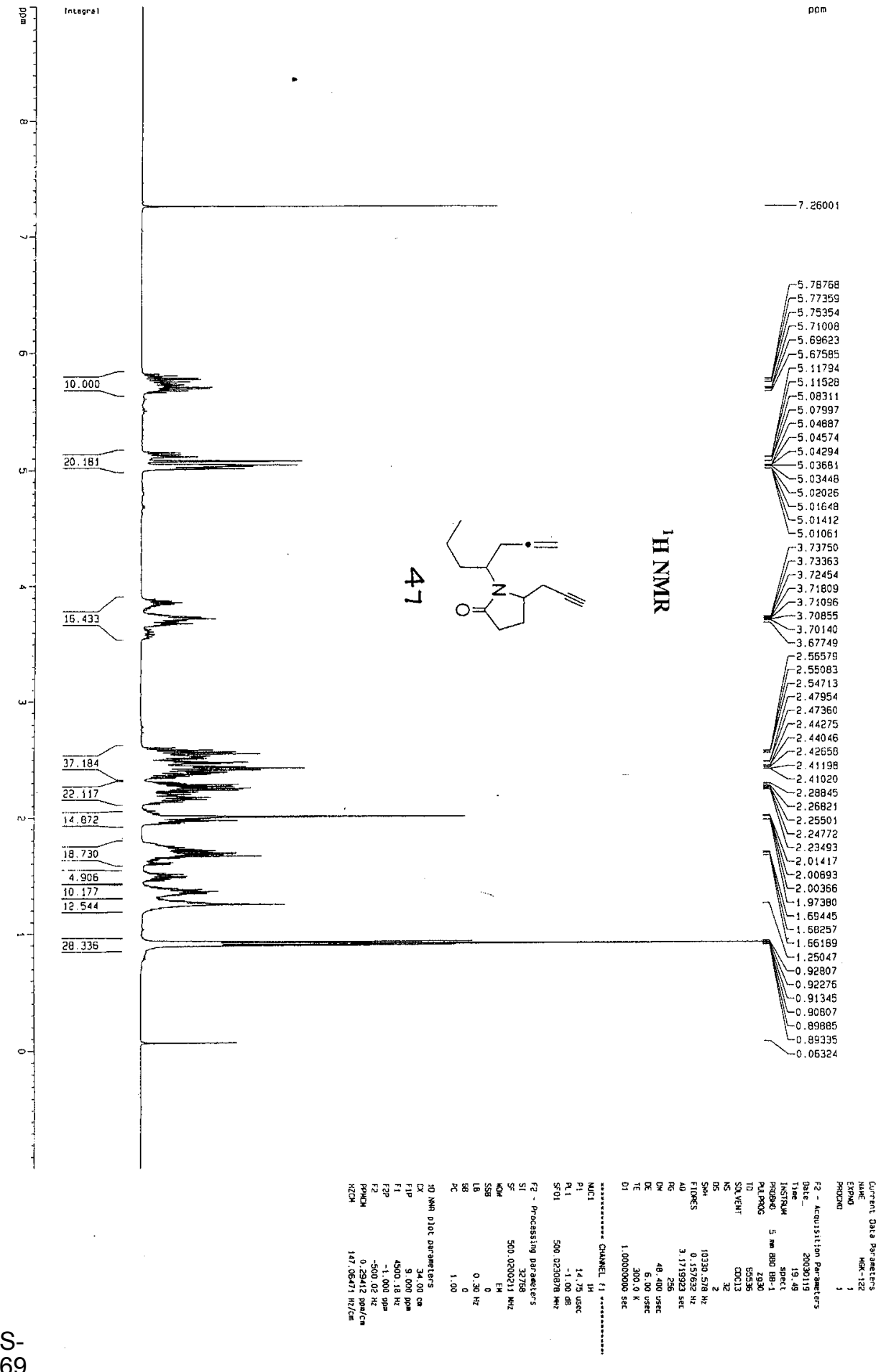




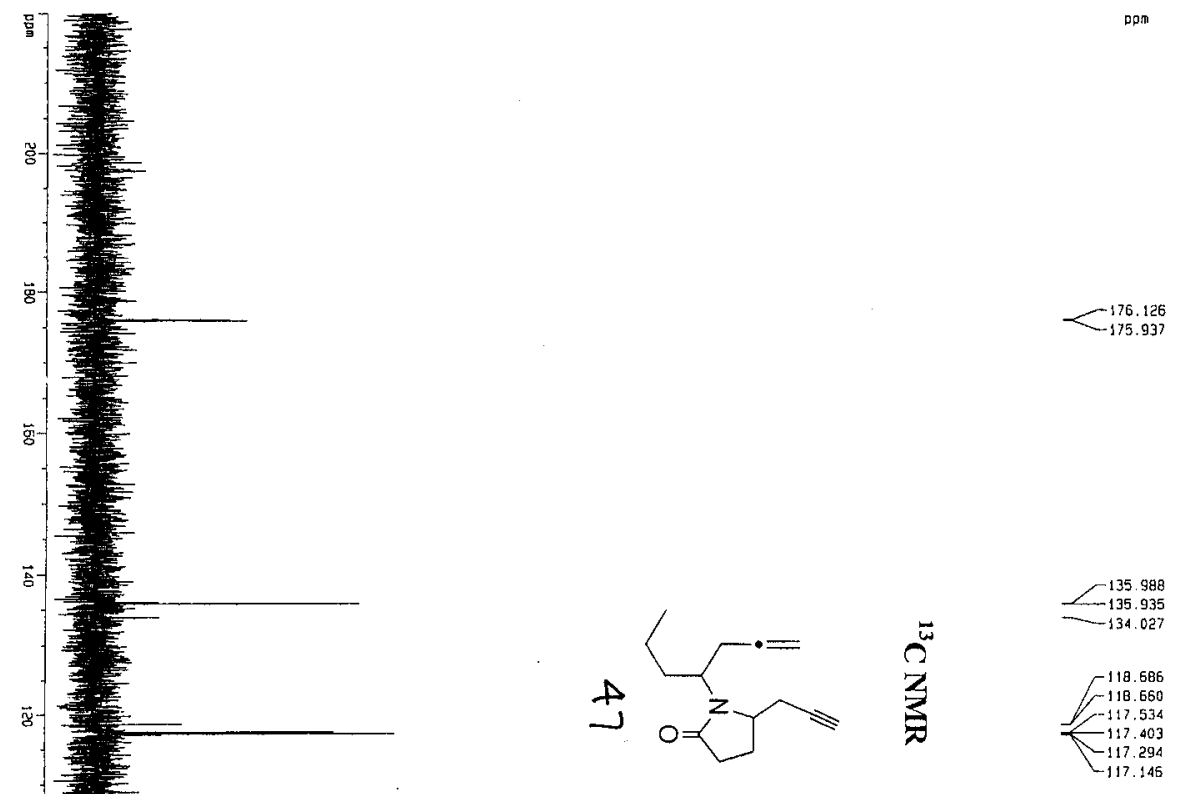

횽

\&.

$-80.552$ $-77.612$ $-77.358$ 77.103 71.284
-71.255 57.236
-56.923 / 53.937 J 53.639 $F_{-}^{39.723}$ 38.800 r 35.999 fi 36.930 If -36.440 Iff 36.440 $\begin{array}{r}34.089 \\ -33.932 \\ \hline\end{array}$ 33.932
-30.870 30.791
30.722 - 30.654 $-25.670$ 25.561
-24.652 L 24.862 L 24.621 - 24.267 20.530 L 20.419 14.320 


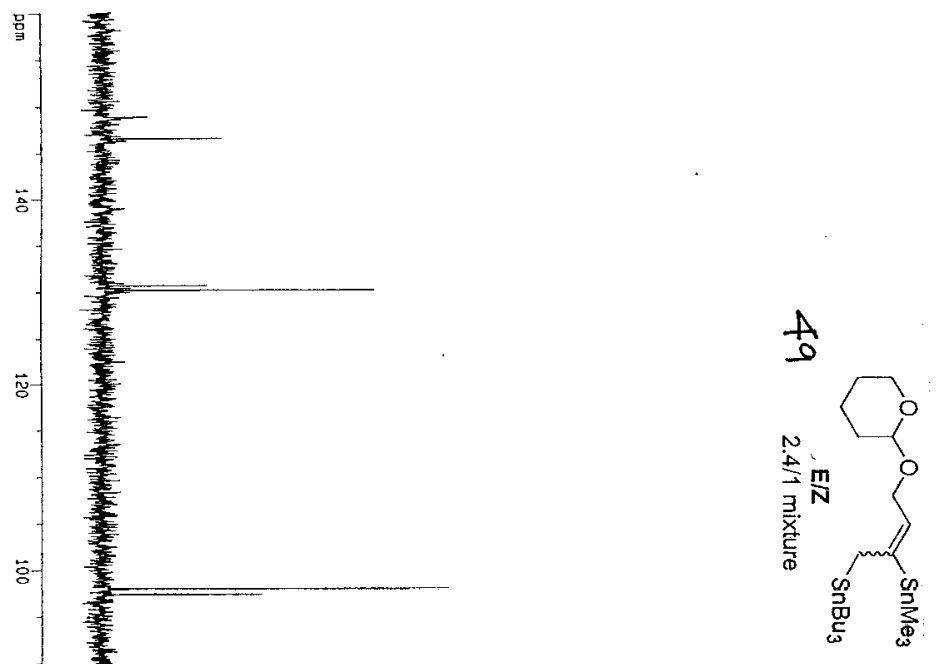

ppm 


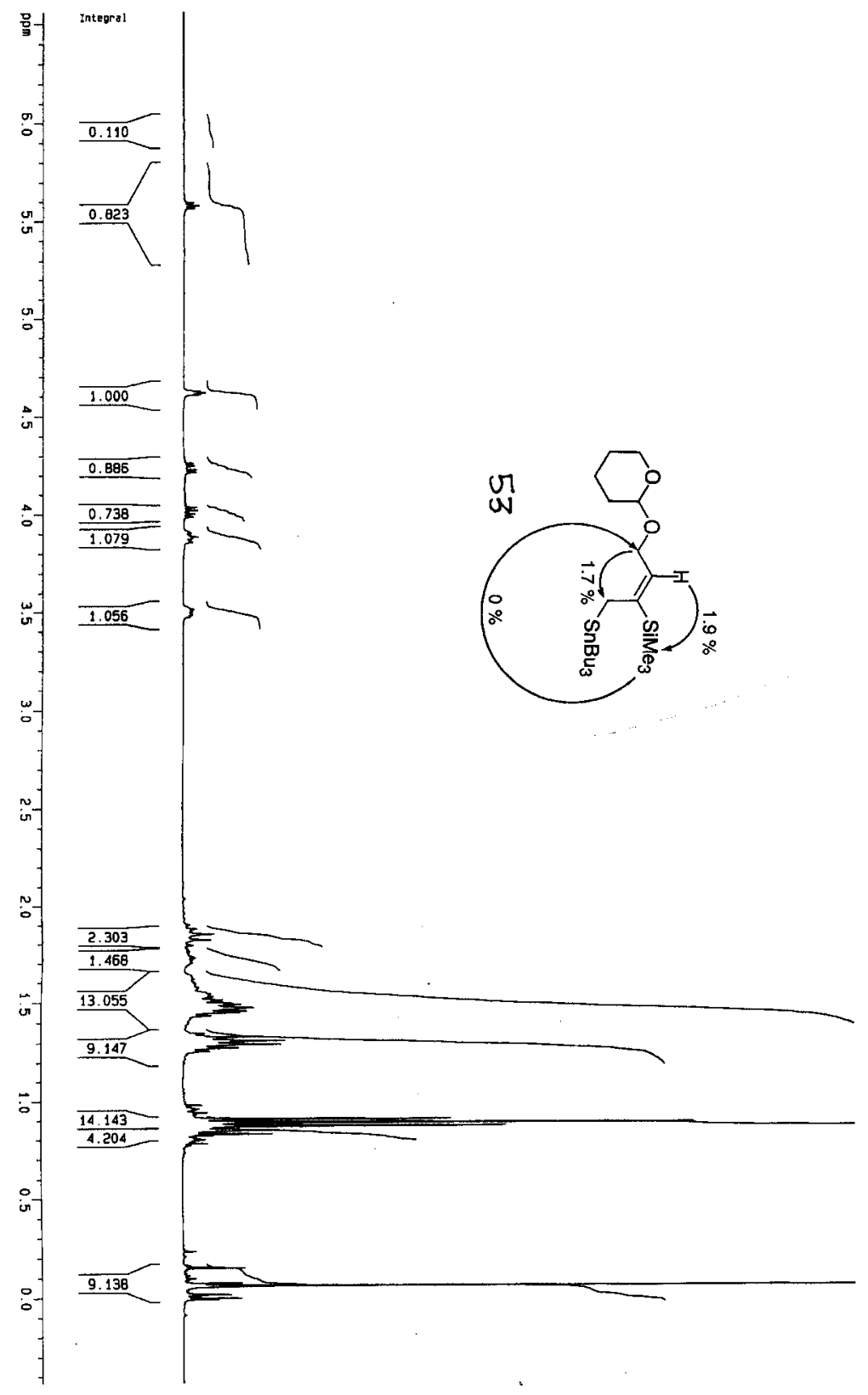




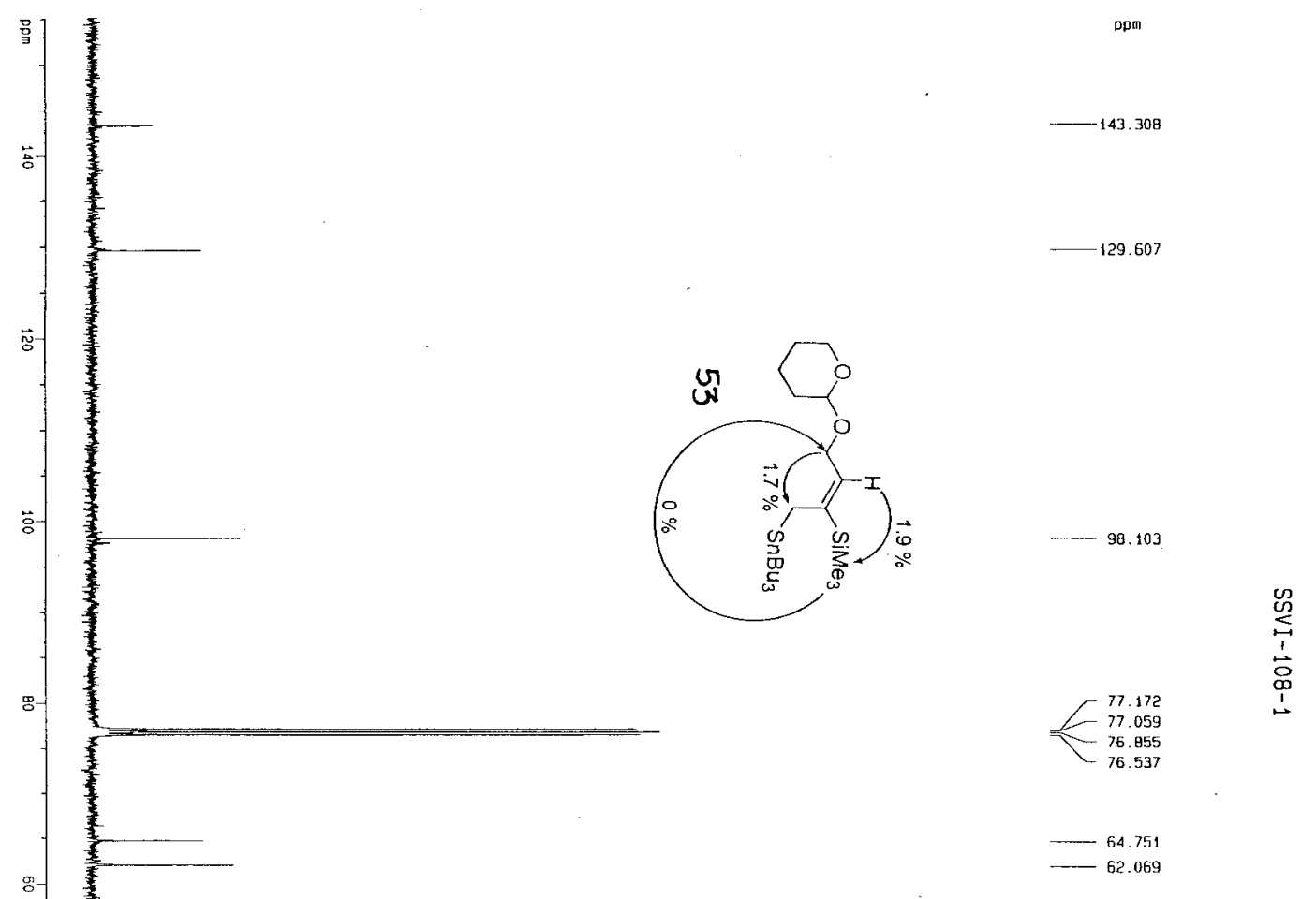

r 30.590

$\Gamma^{30.590} 30.4 \mathrm{A3}$

29.124

29.040

28.963

28. 867

$-27.323$

- 27.269

25.386

$-13.520$

$=\quad 12.887$

- 9.905

9.823

8. 602

f

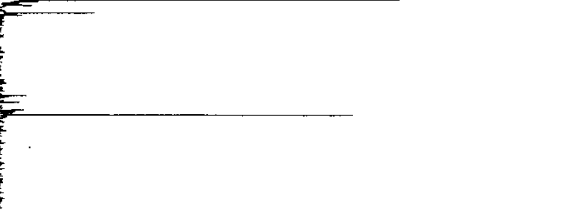

S- 

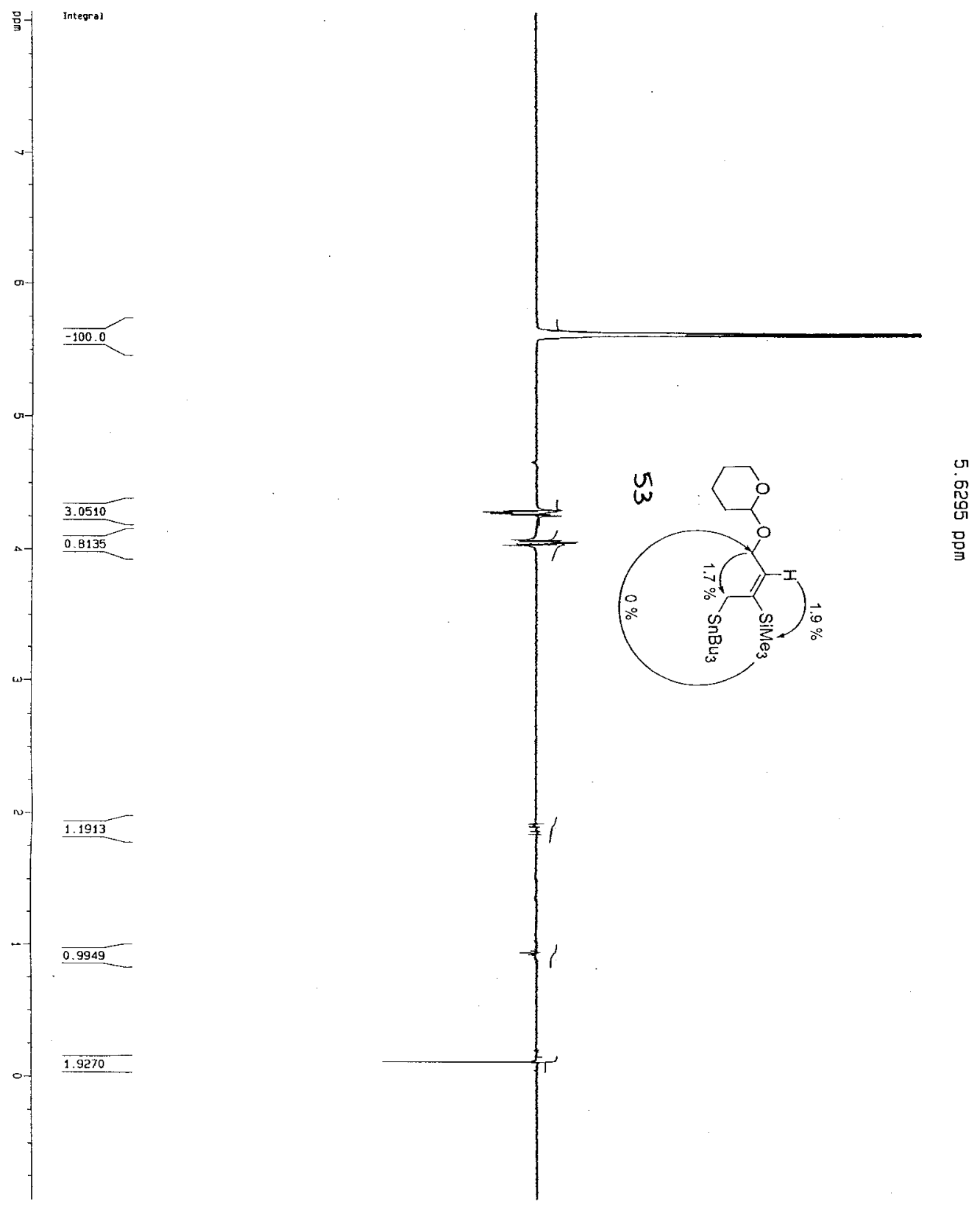



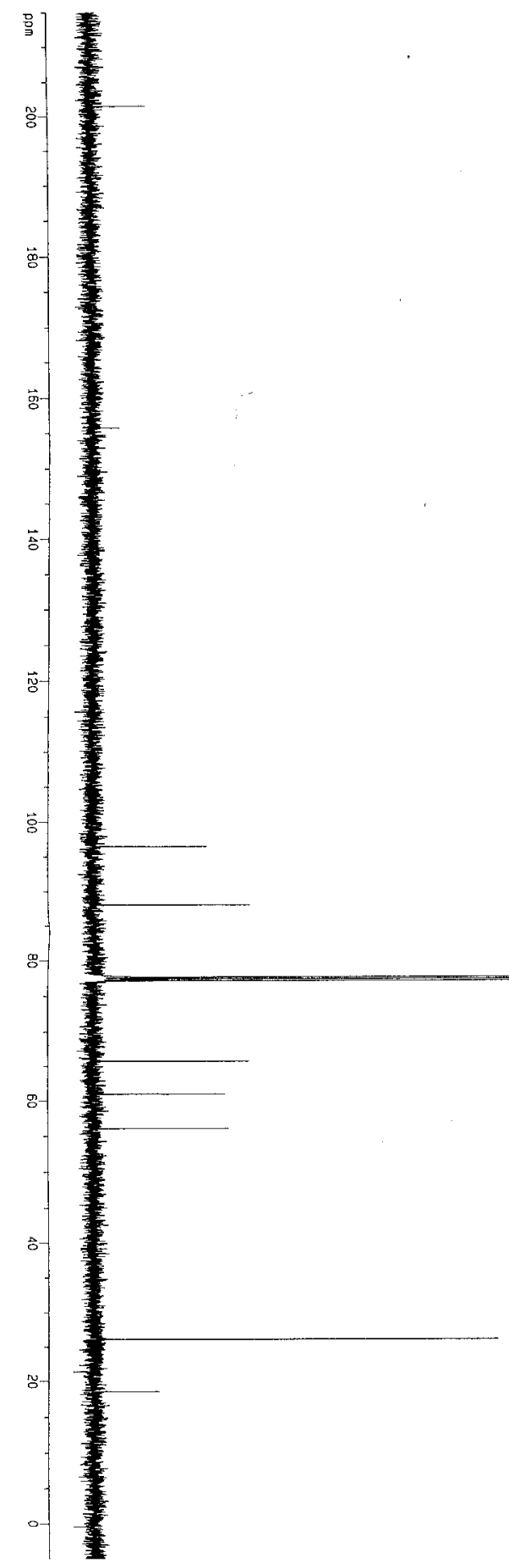

$-201.547$

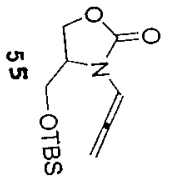

88.085 


$$
\text { 地 }
$$




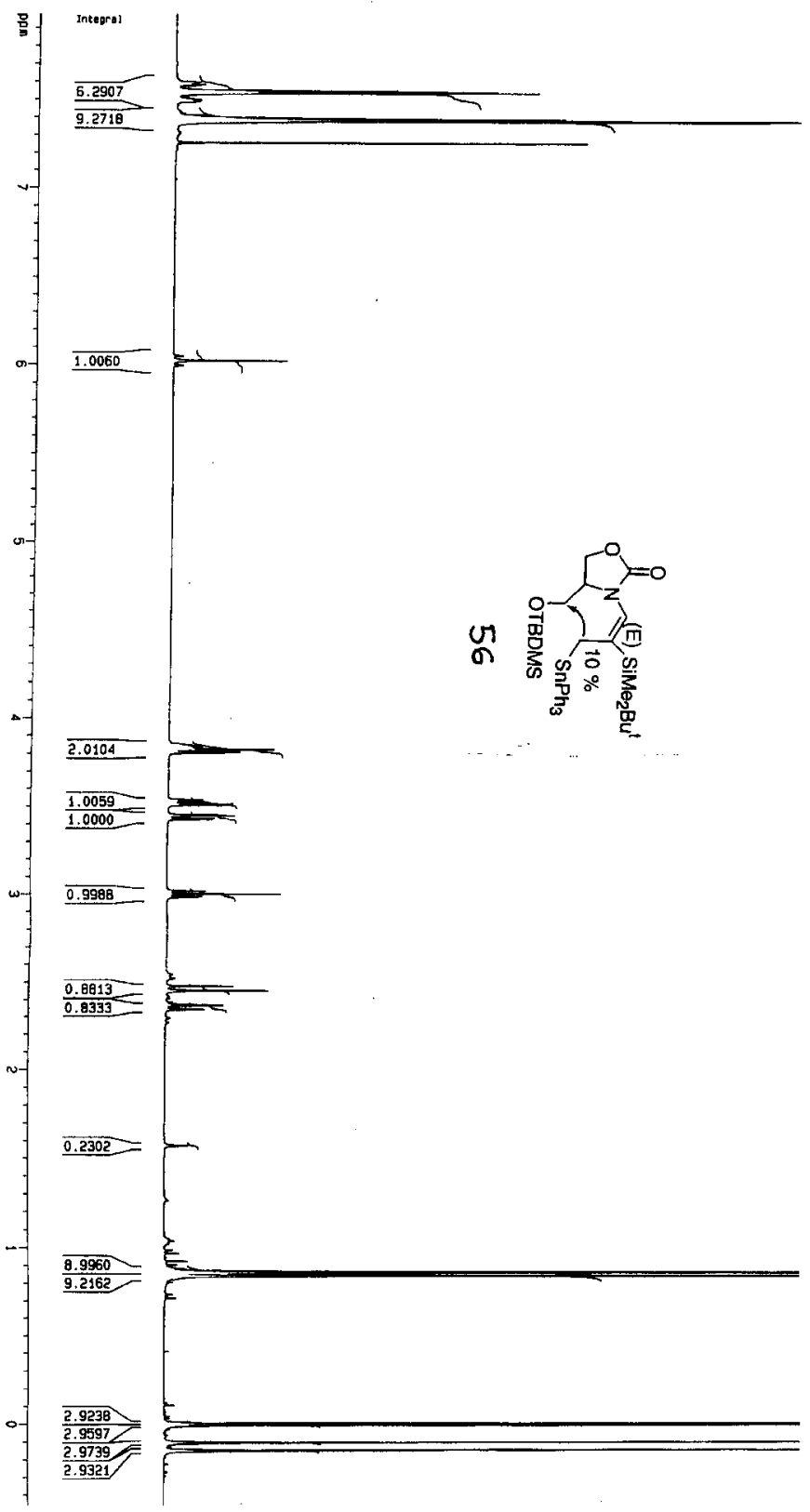




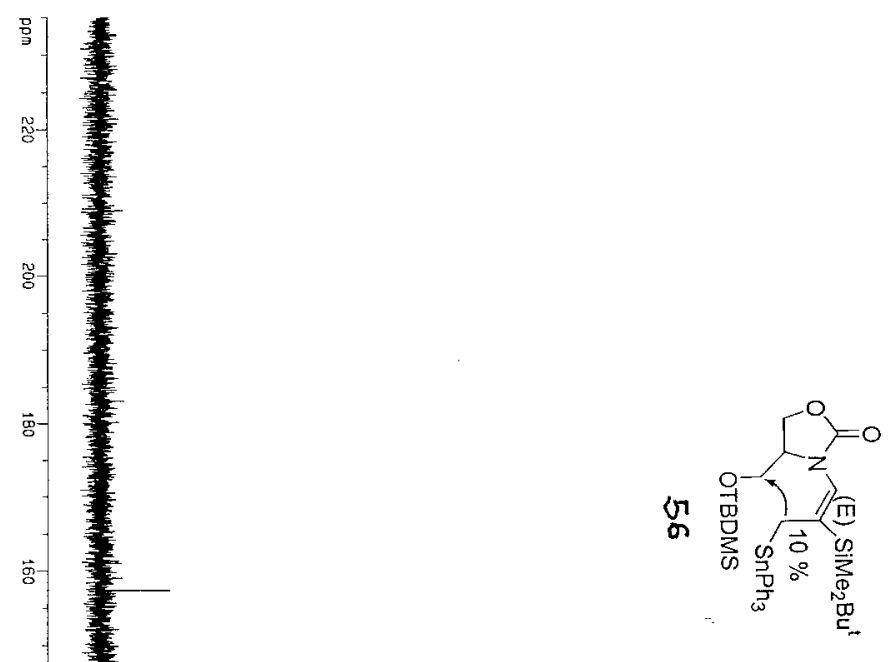

$-139.055$ 139.055
-137.616
-137.476 $-137.337$ $-129.530$ $-129.244$

$-129.051$

$-128.855$ $-126.774$ $\$ 26.569$ 


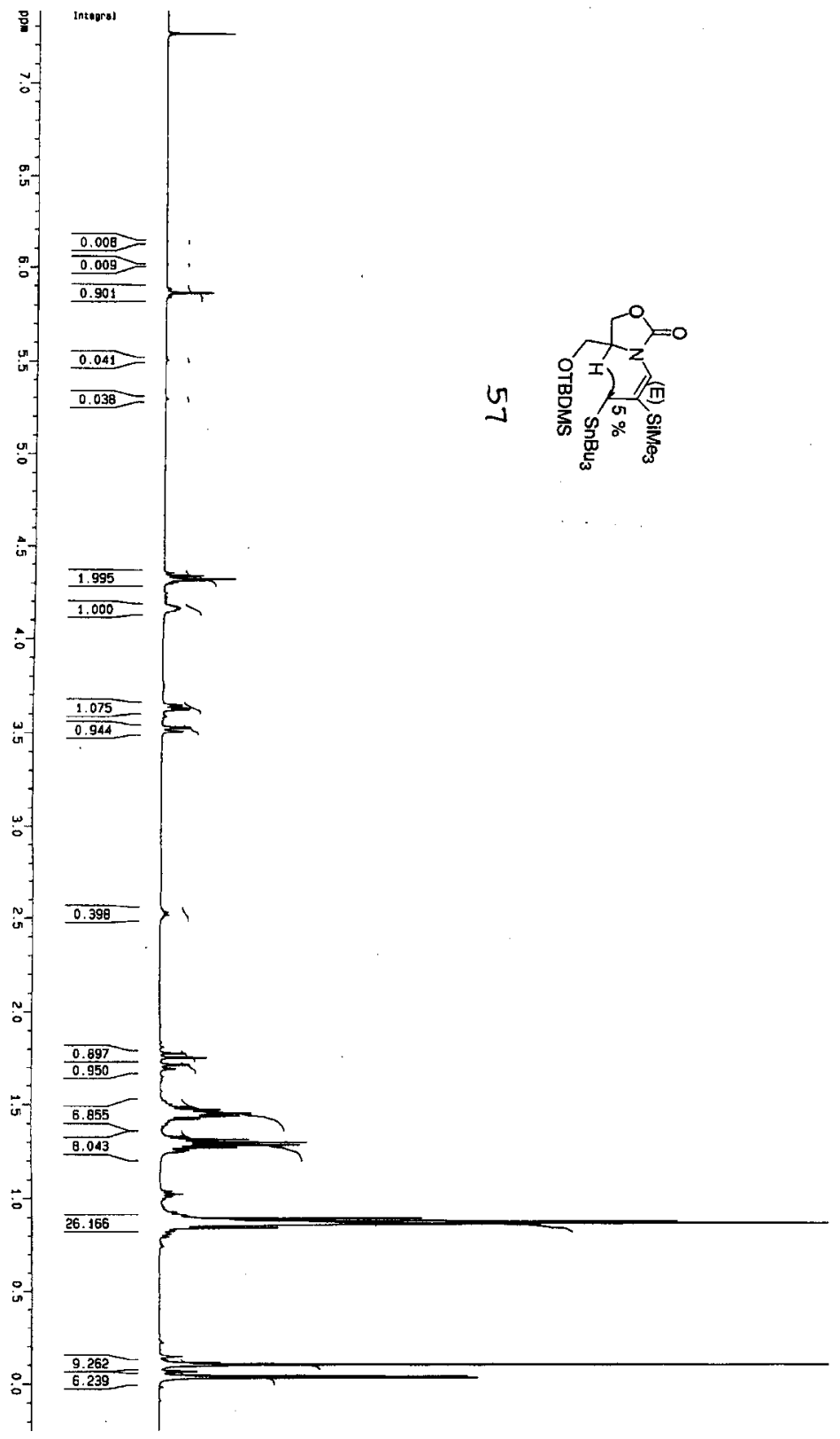



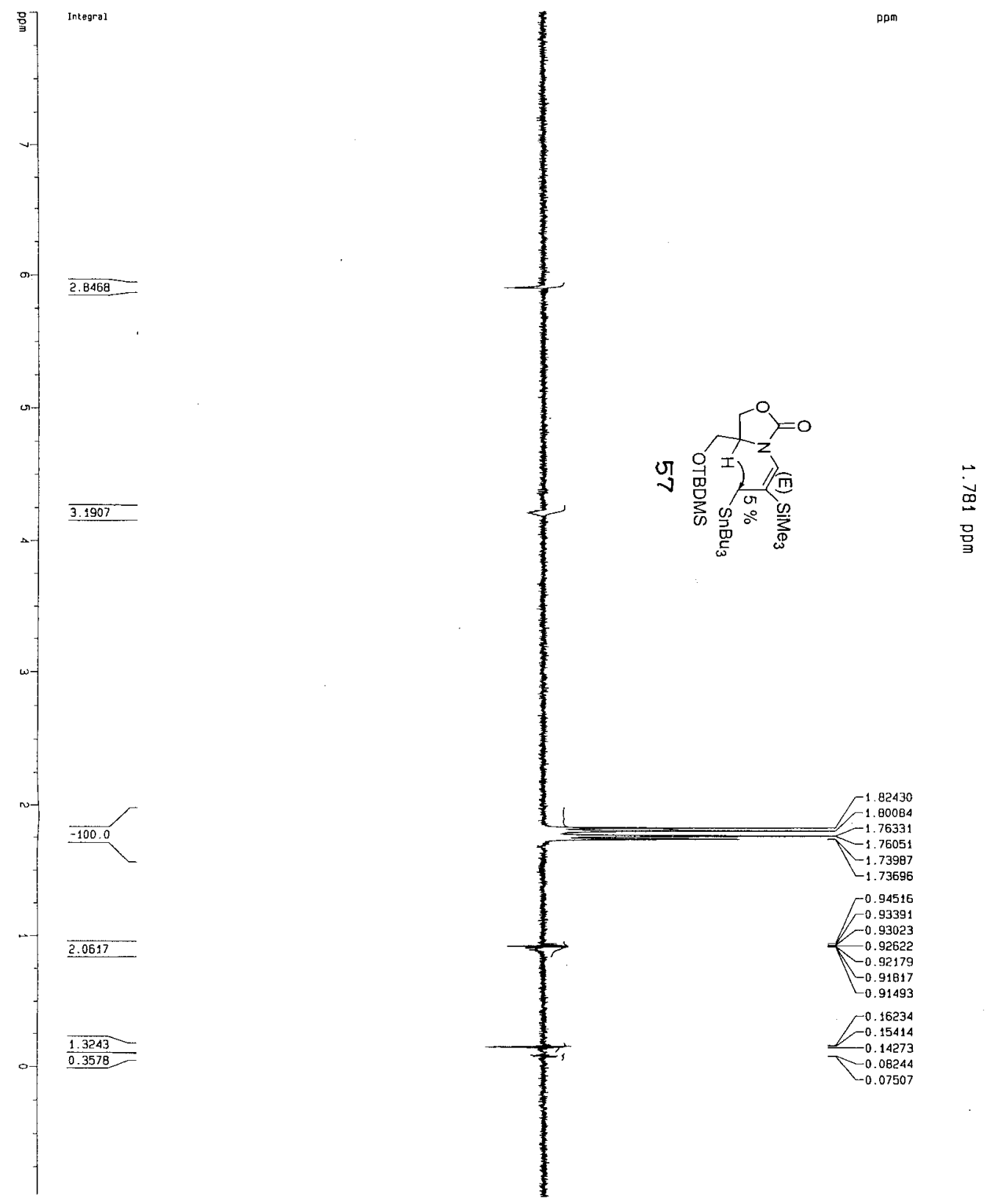


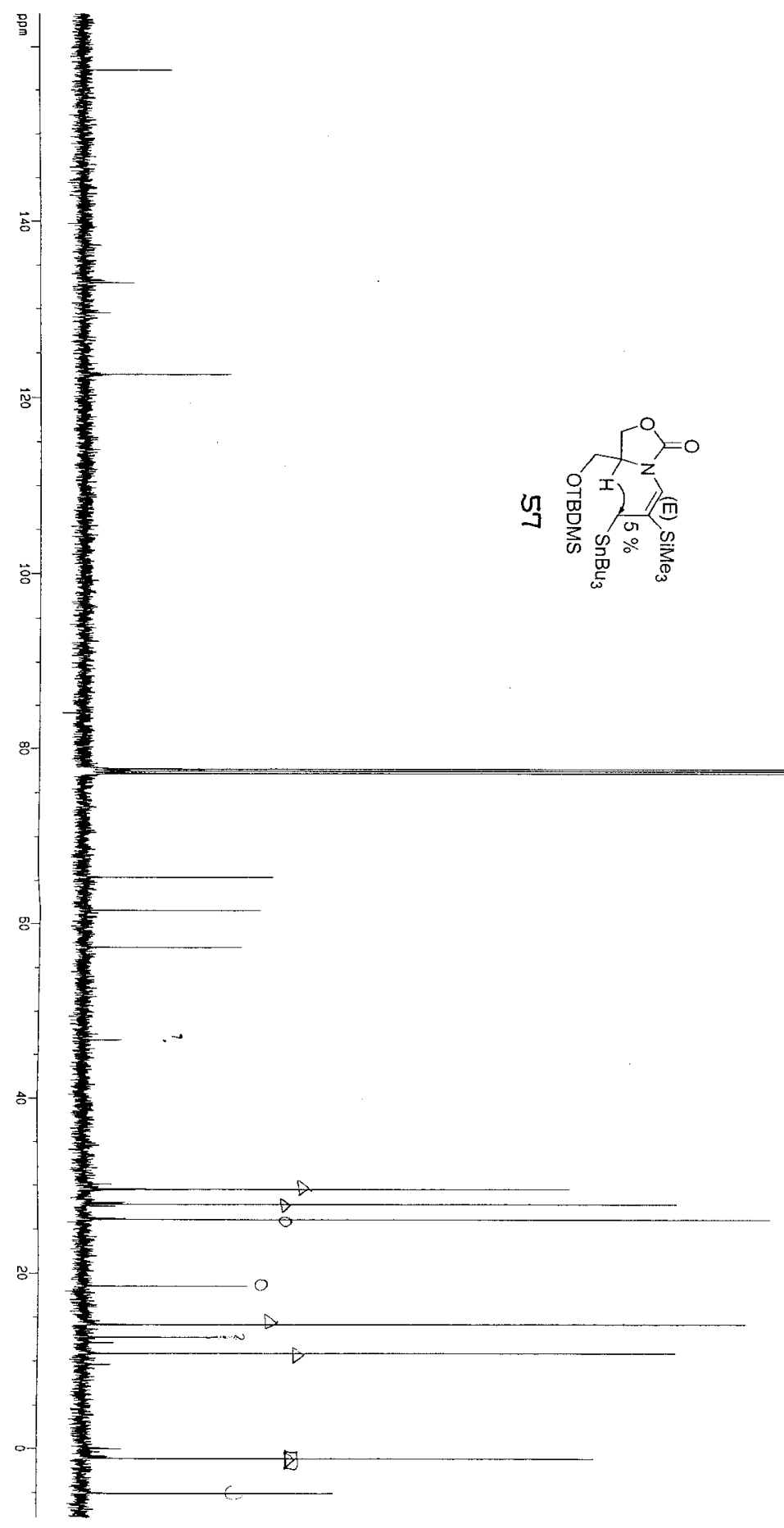



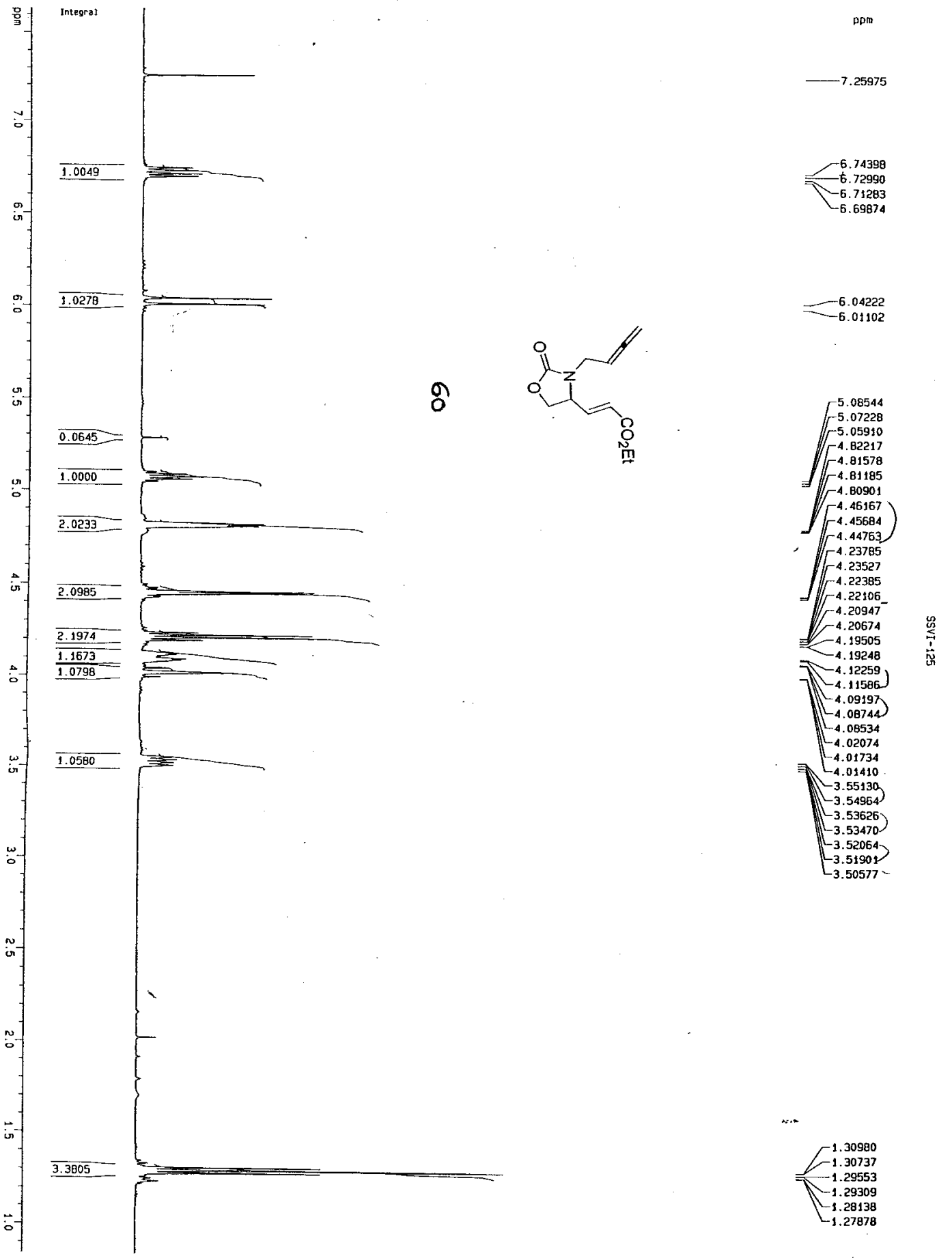


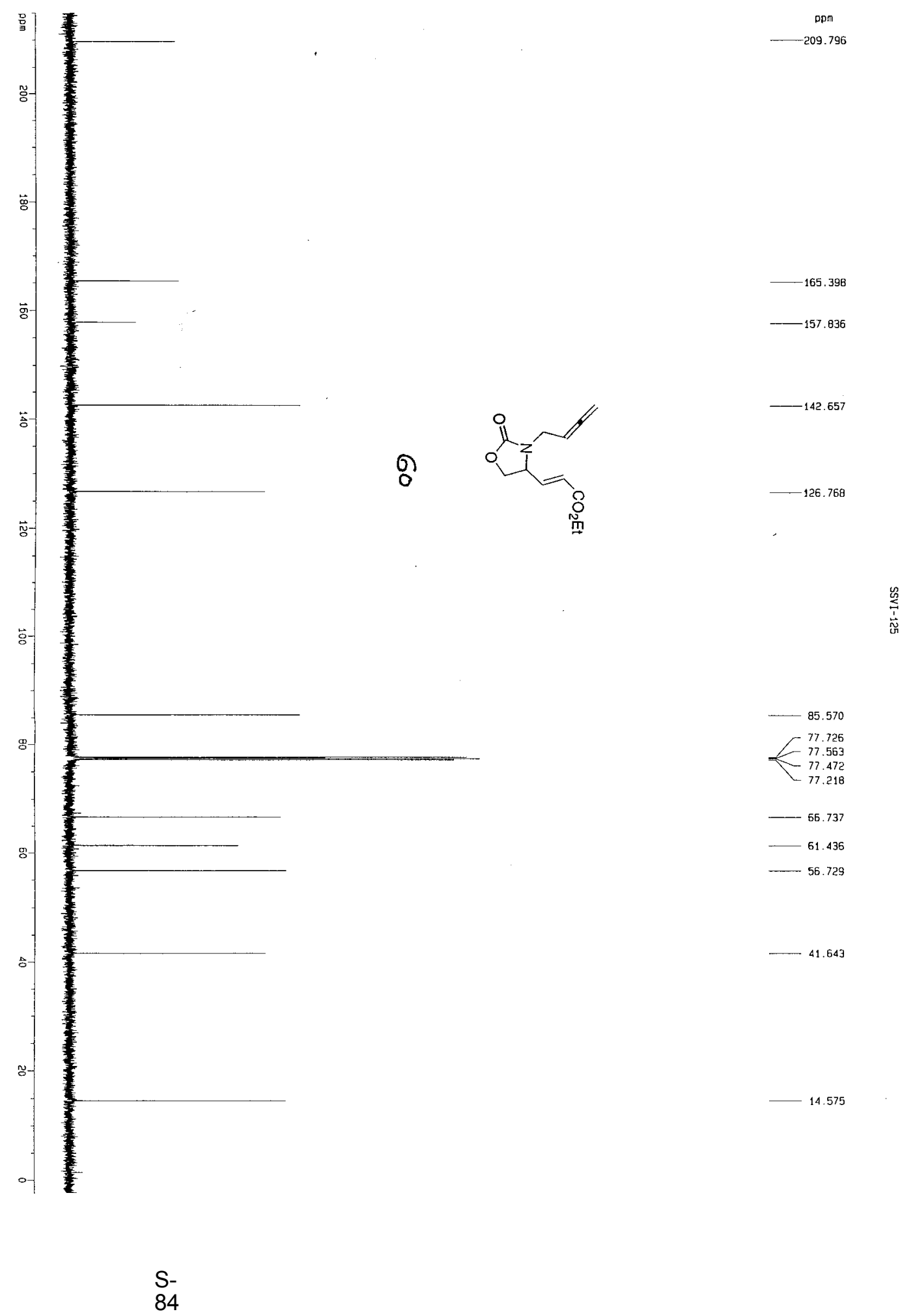




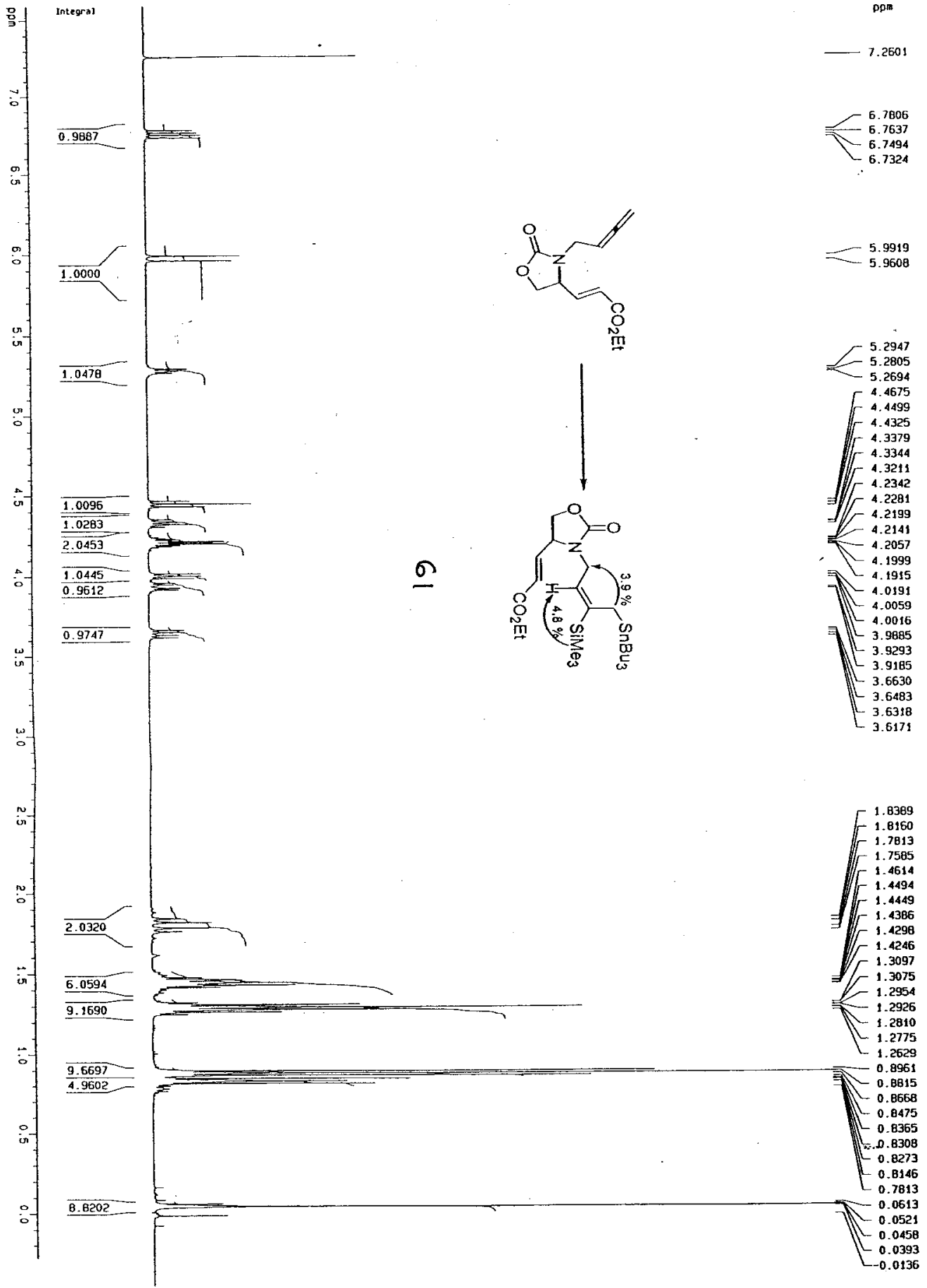



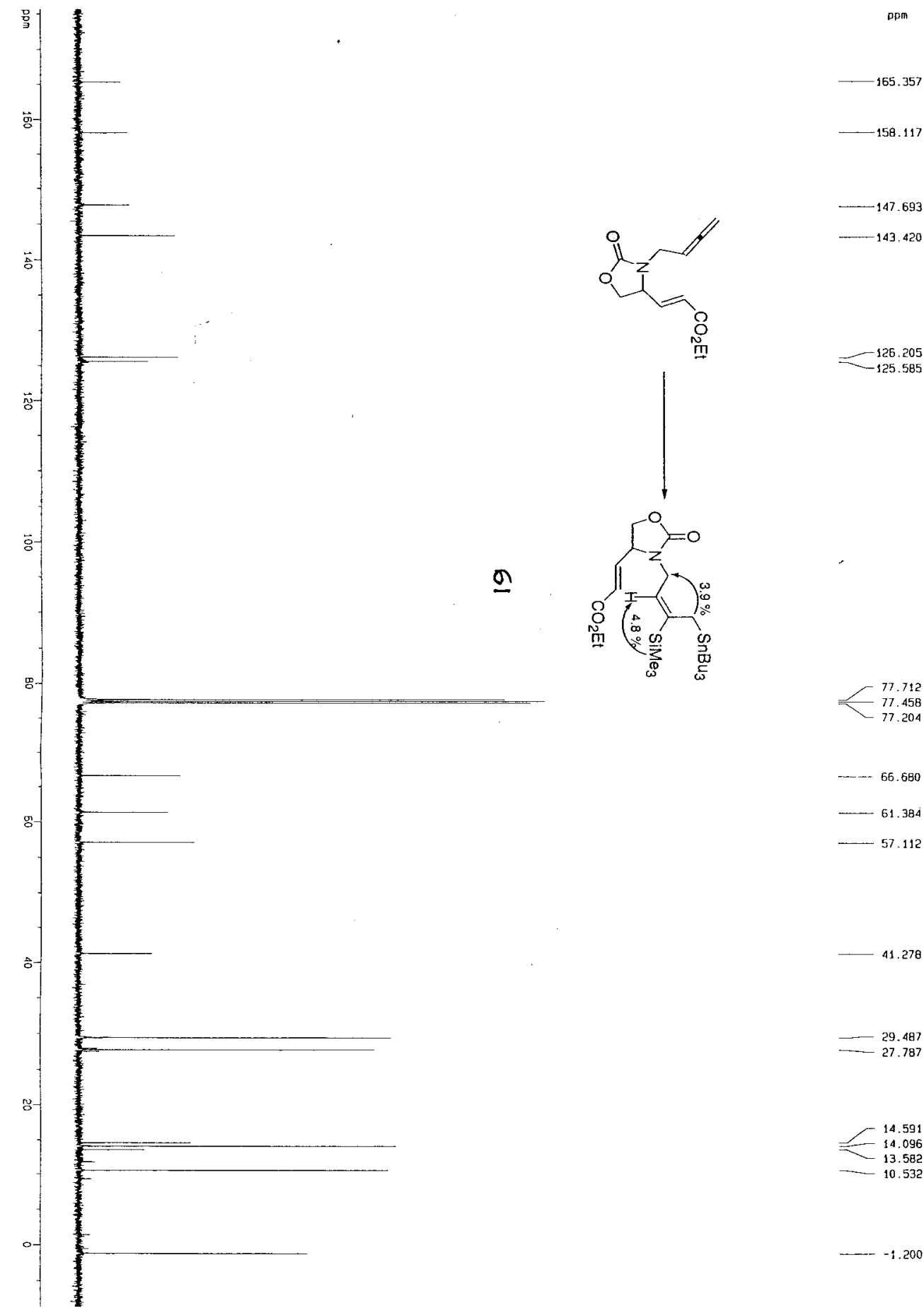

$-143.420$
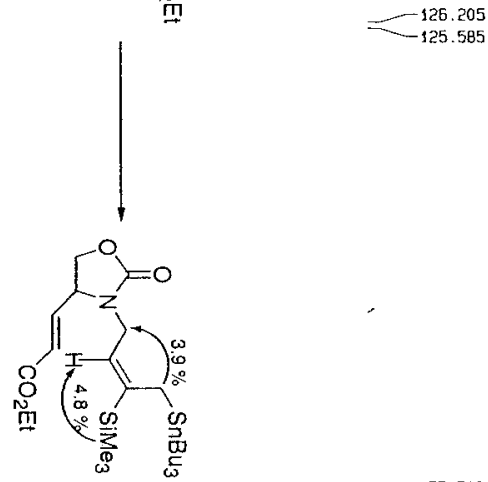

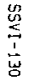

77.712 77. 458

$-77.204$

$-61.384$

57.112

41. 278

$-29.487$

27.787

14.591

14. 096

13.582

10.532

S-

86 


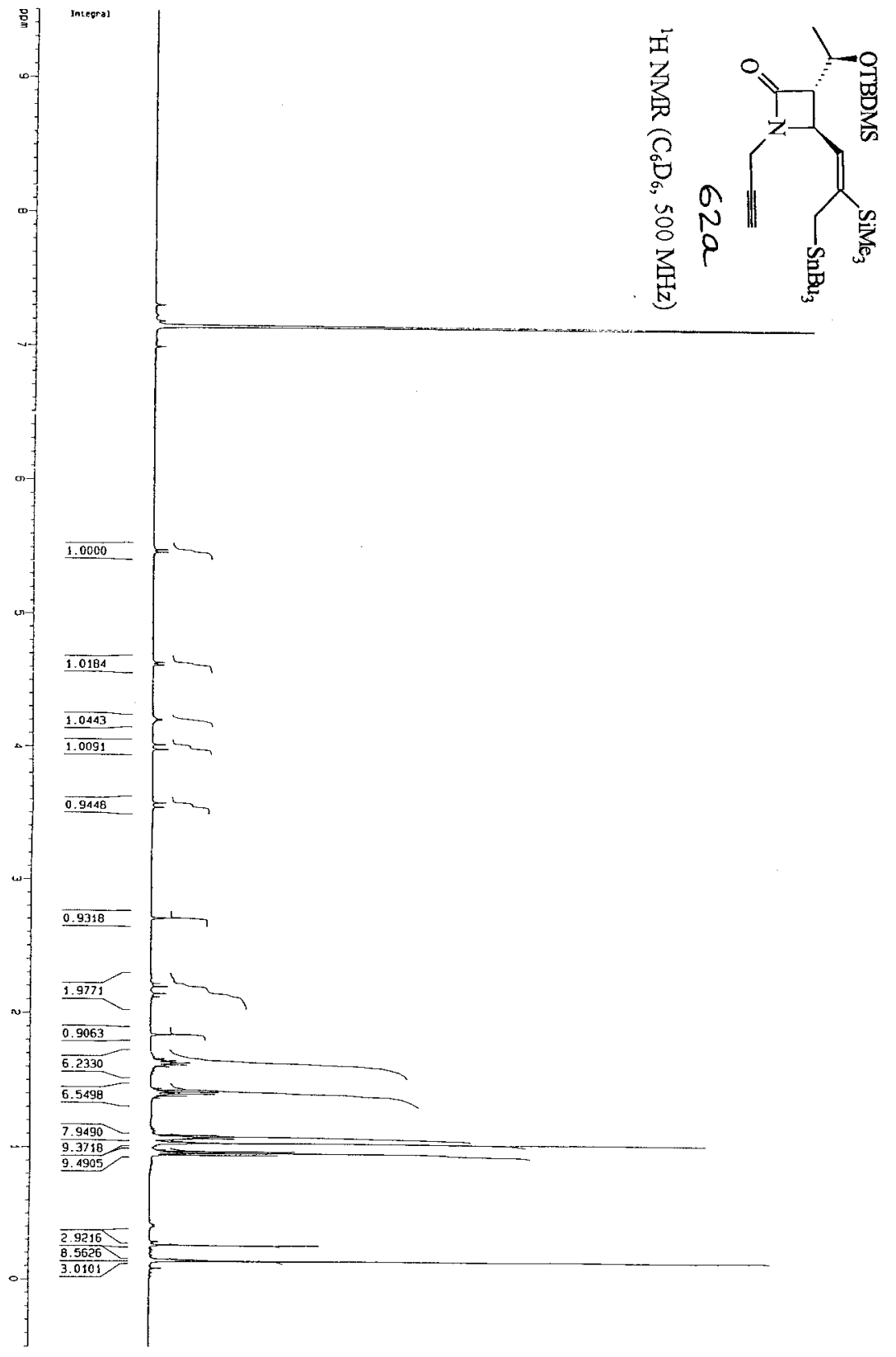




$$
E^{\prime \prime}
$$




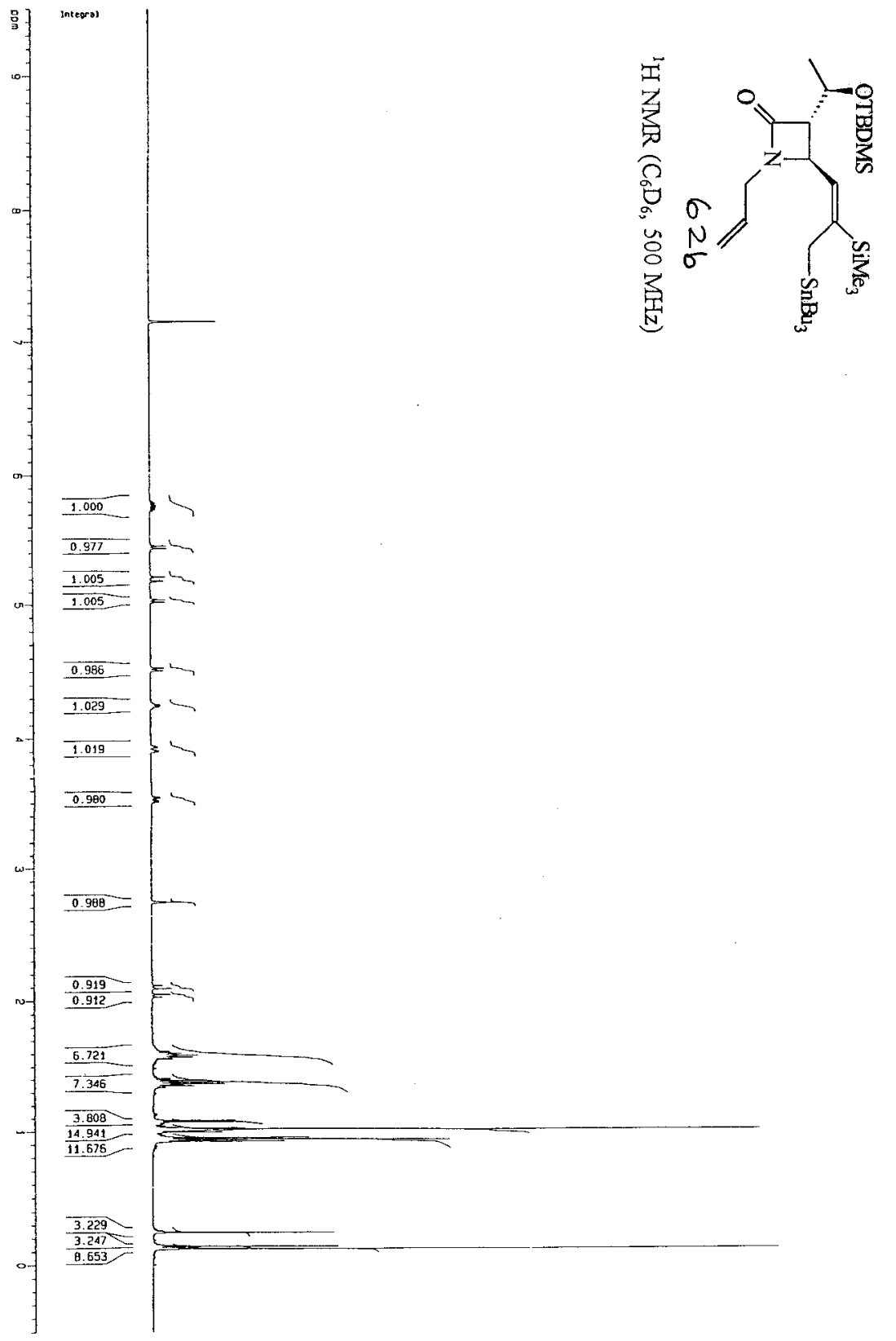

S-

89 


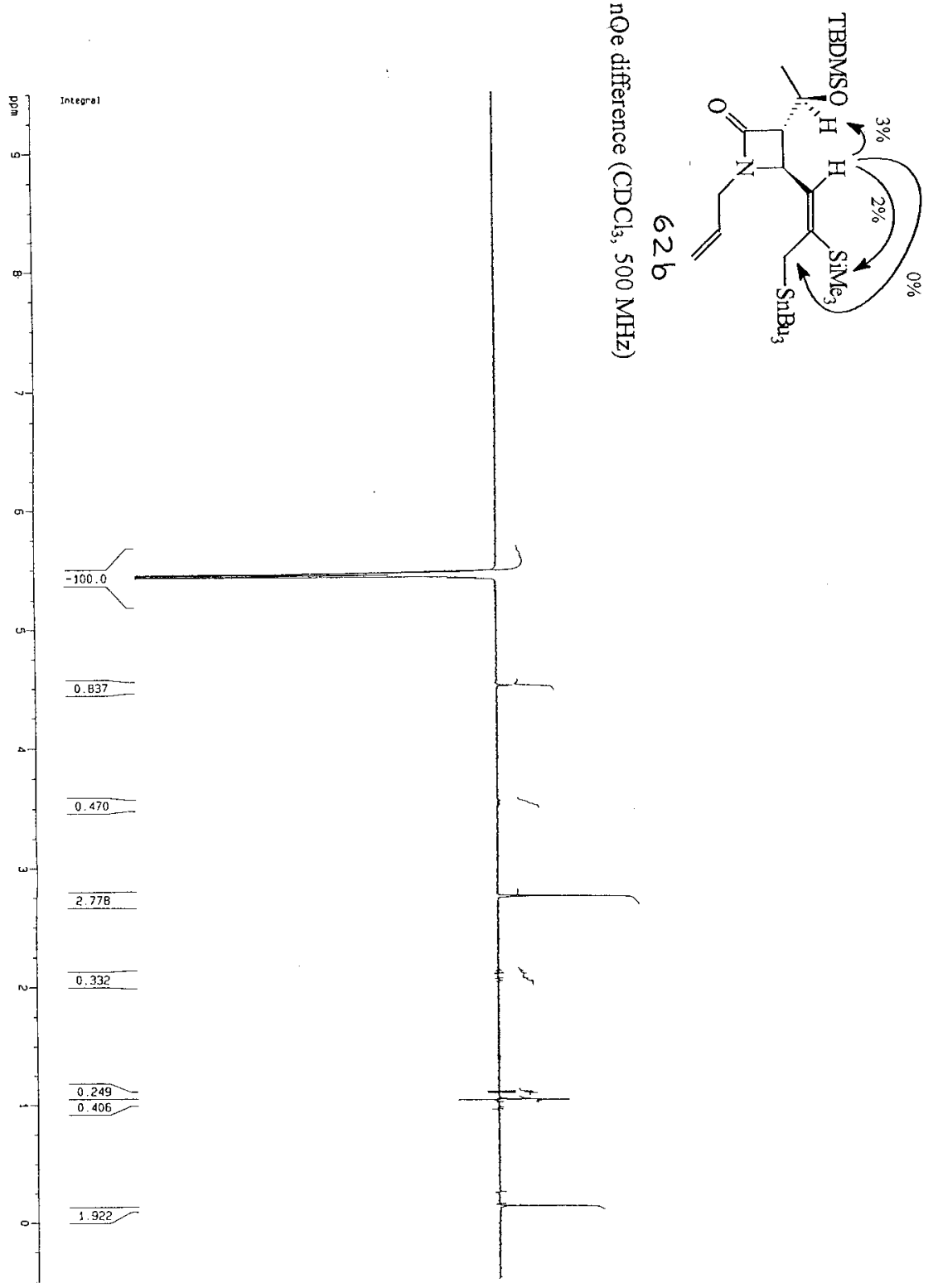

S-

90 


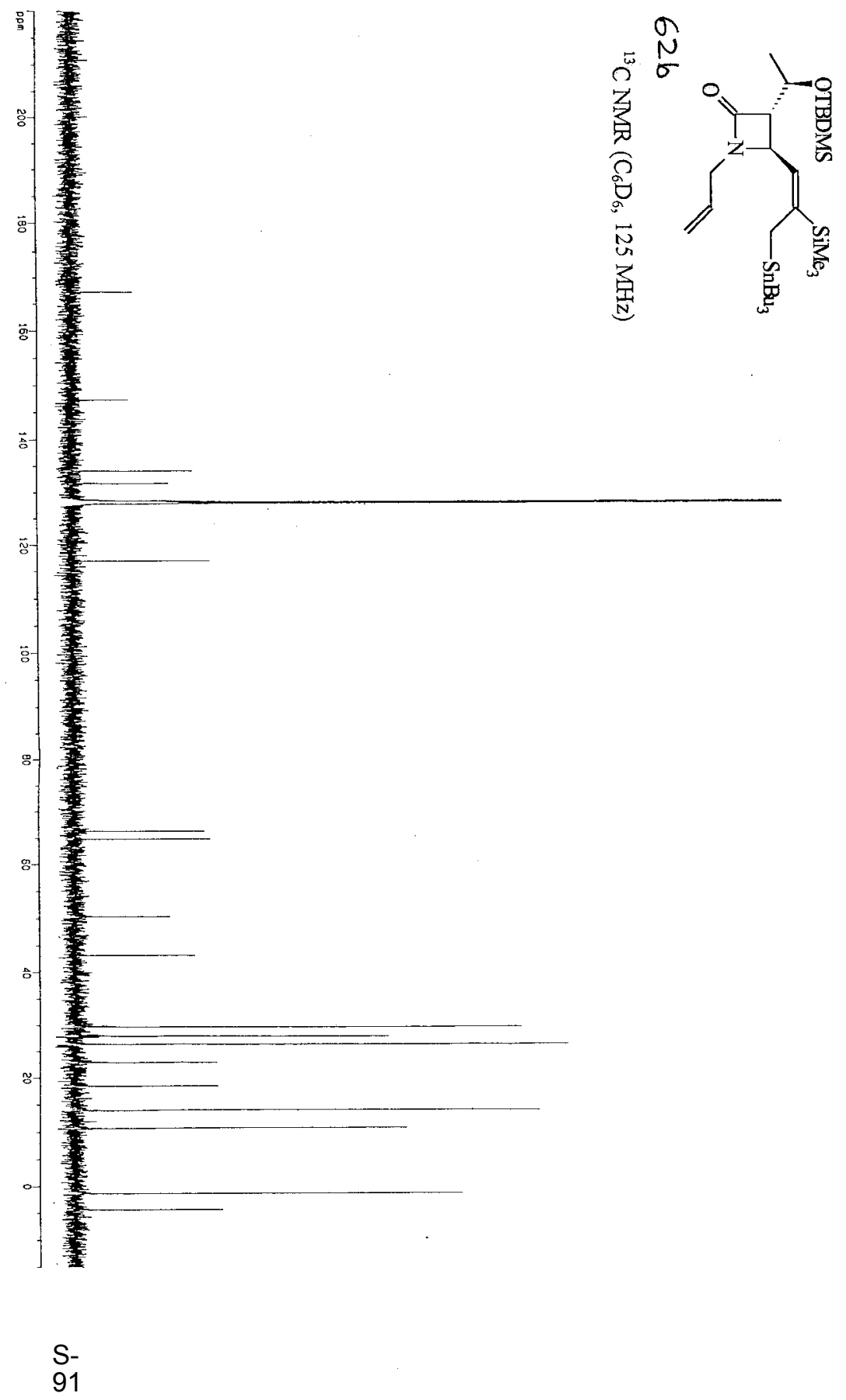




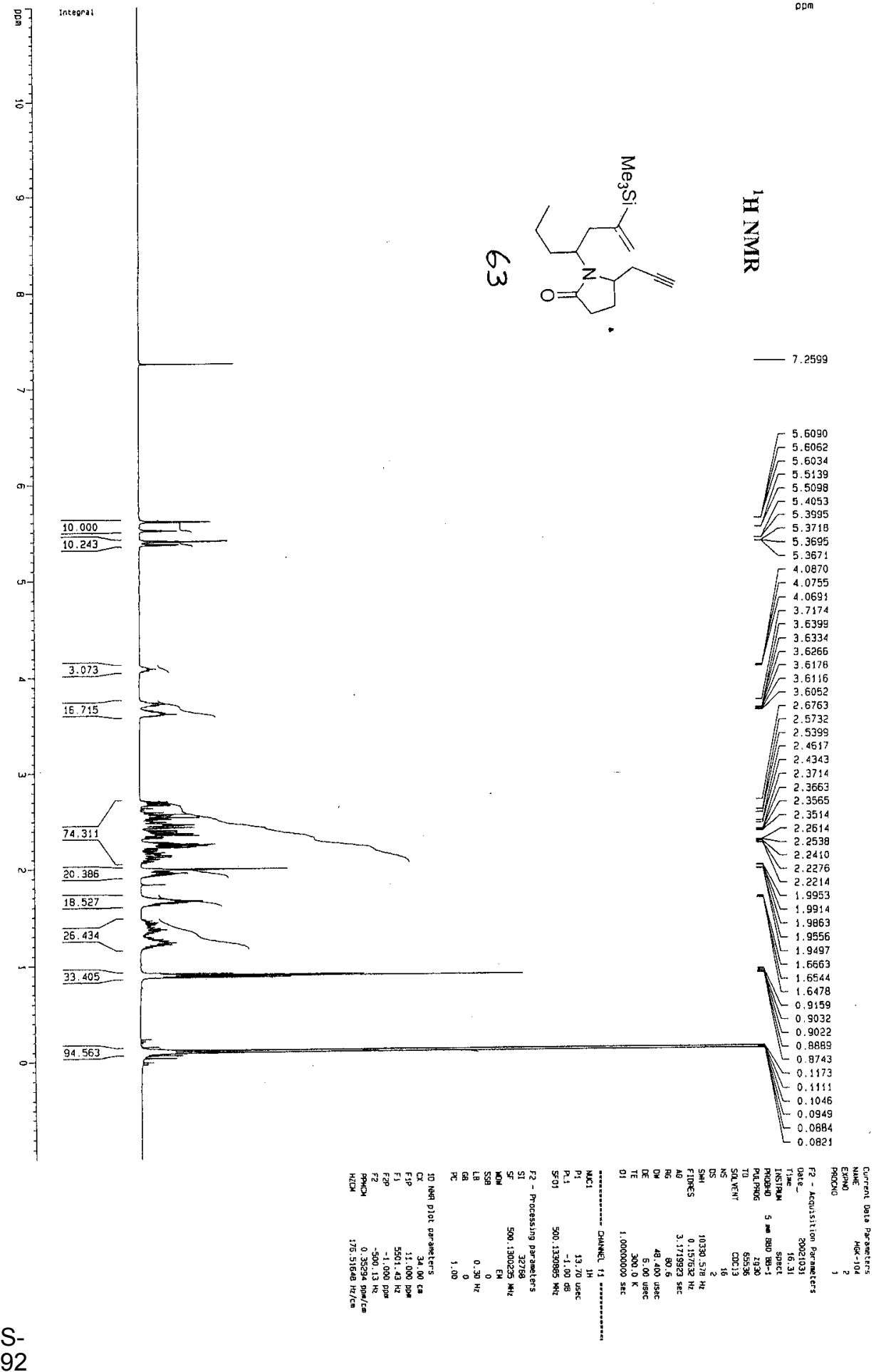




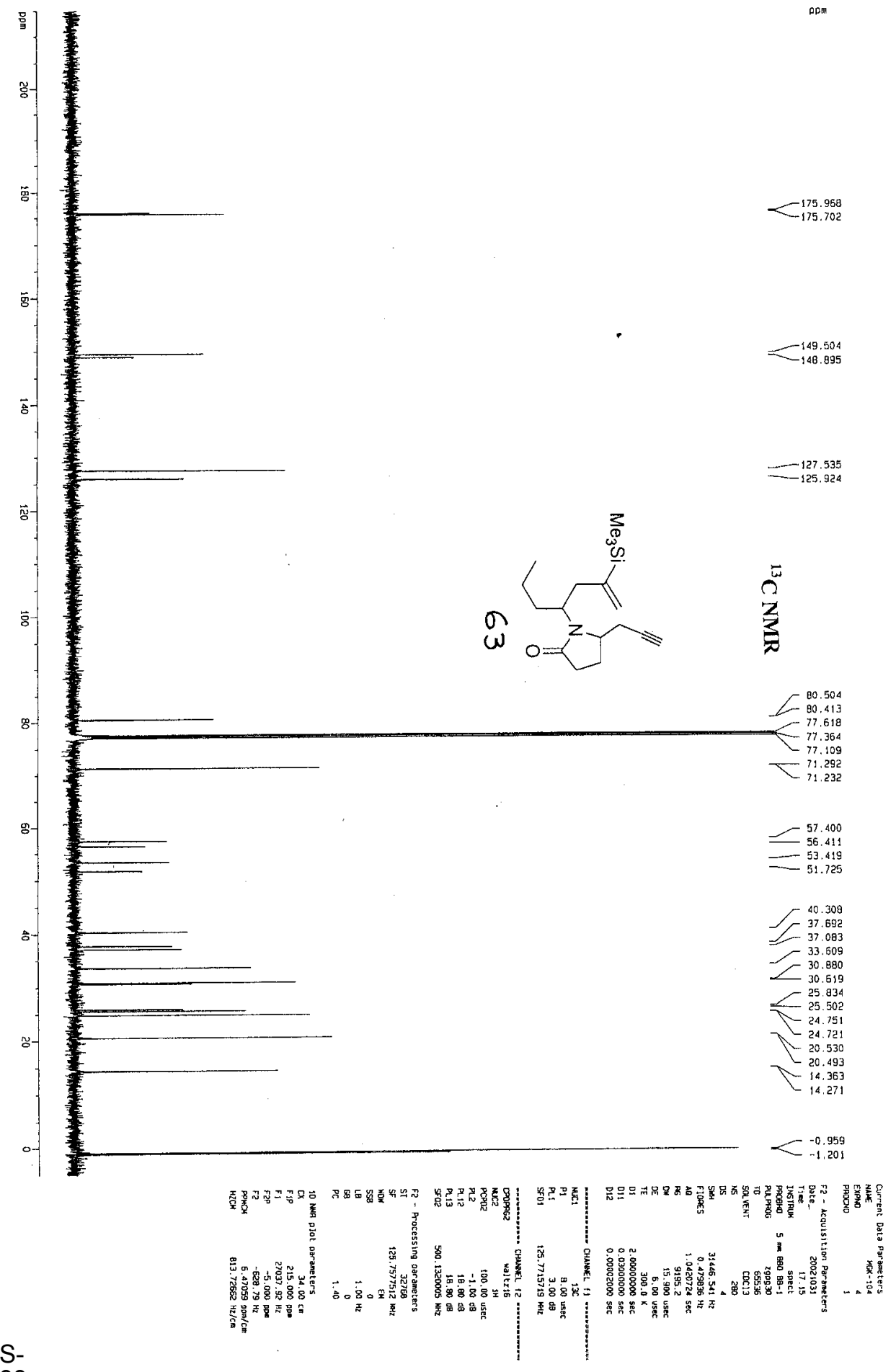




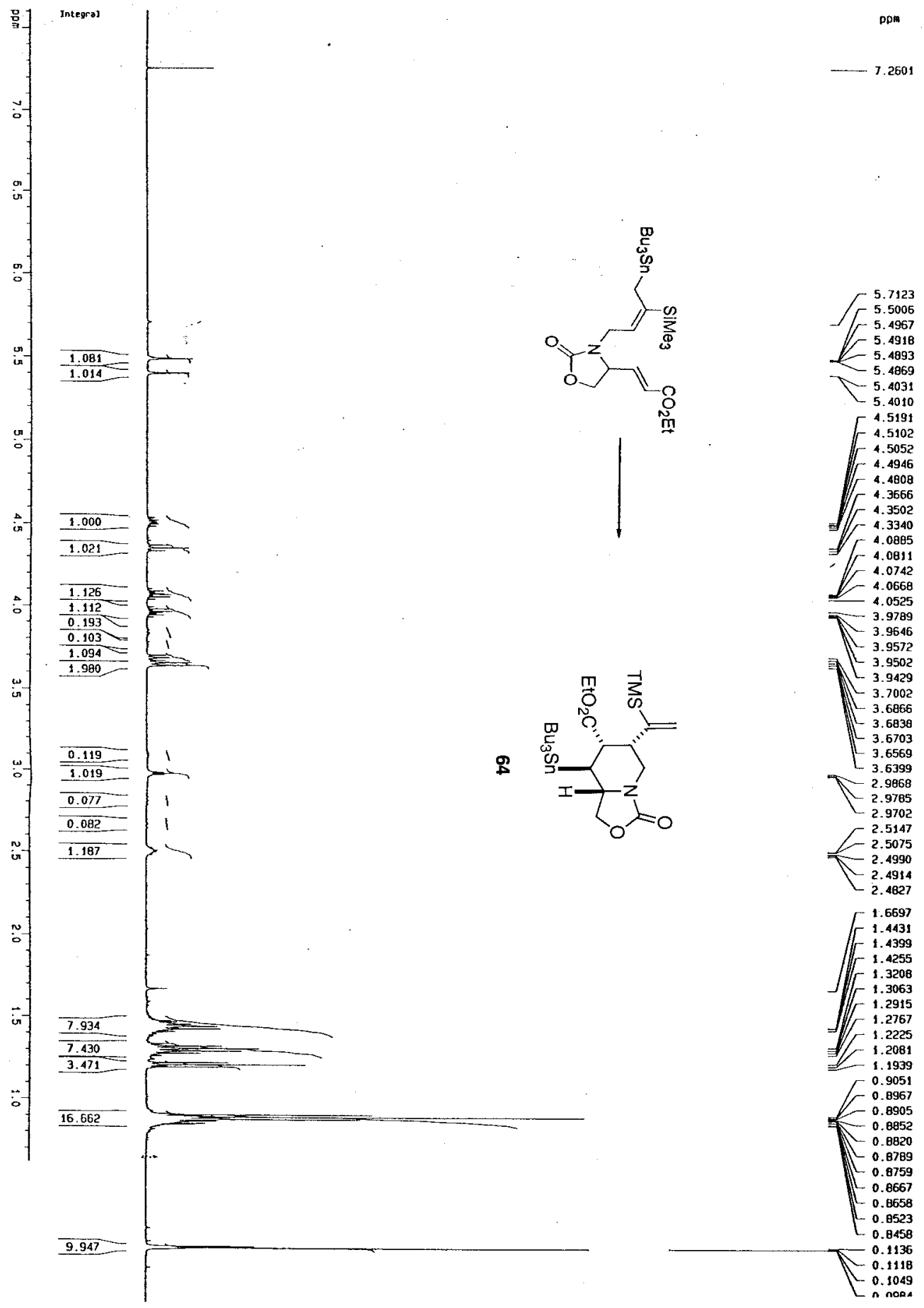




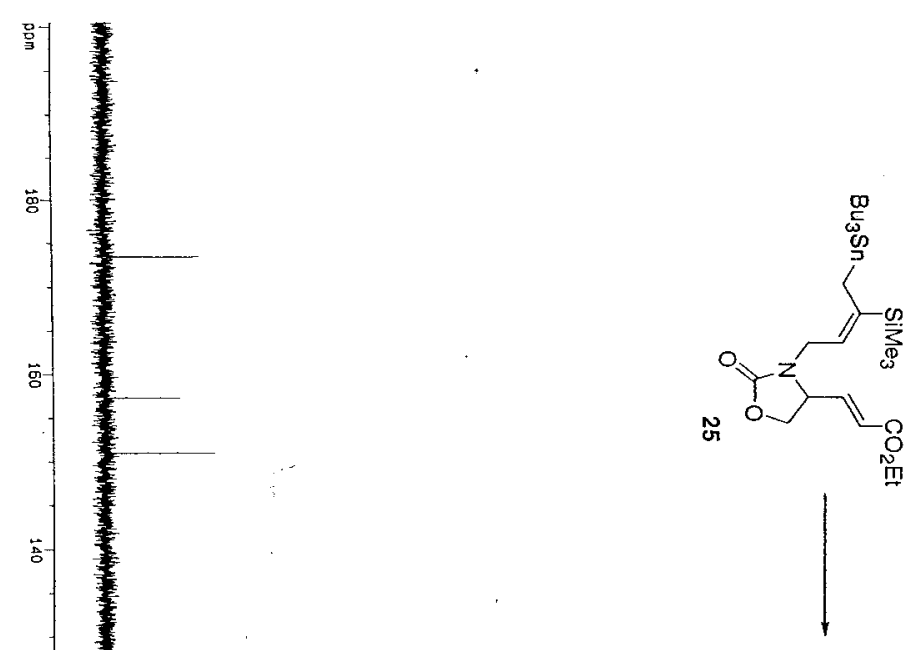

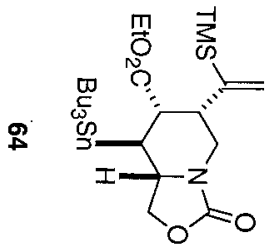
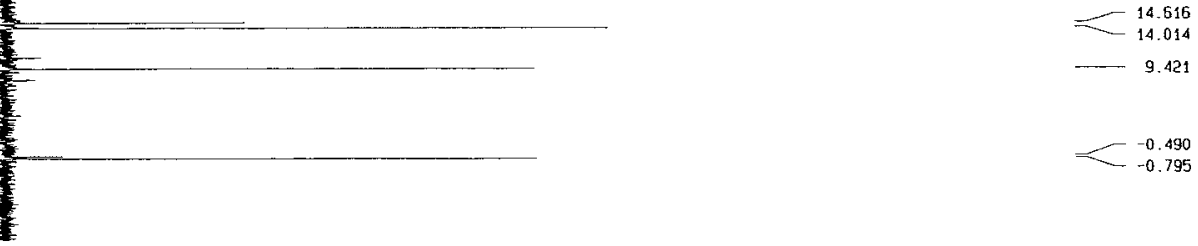

S- 


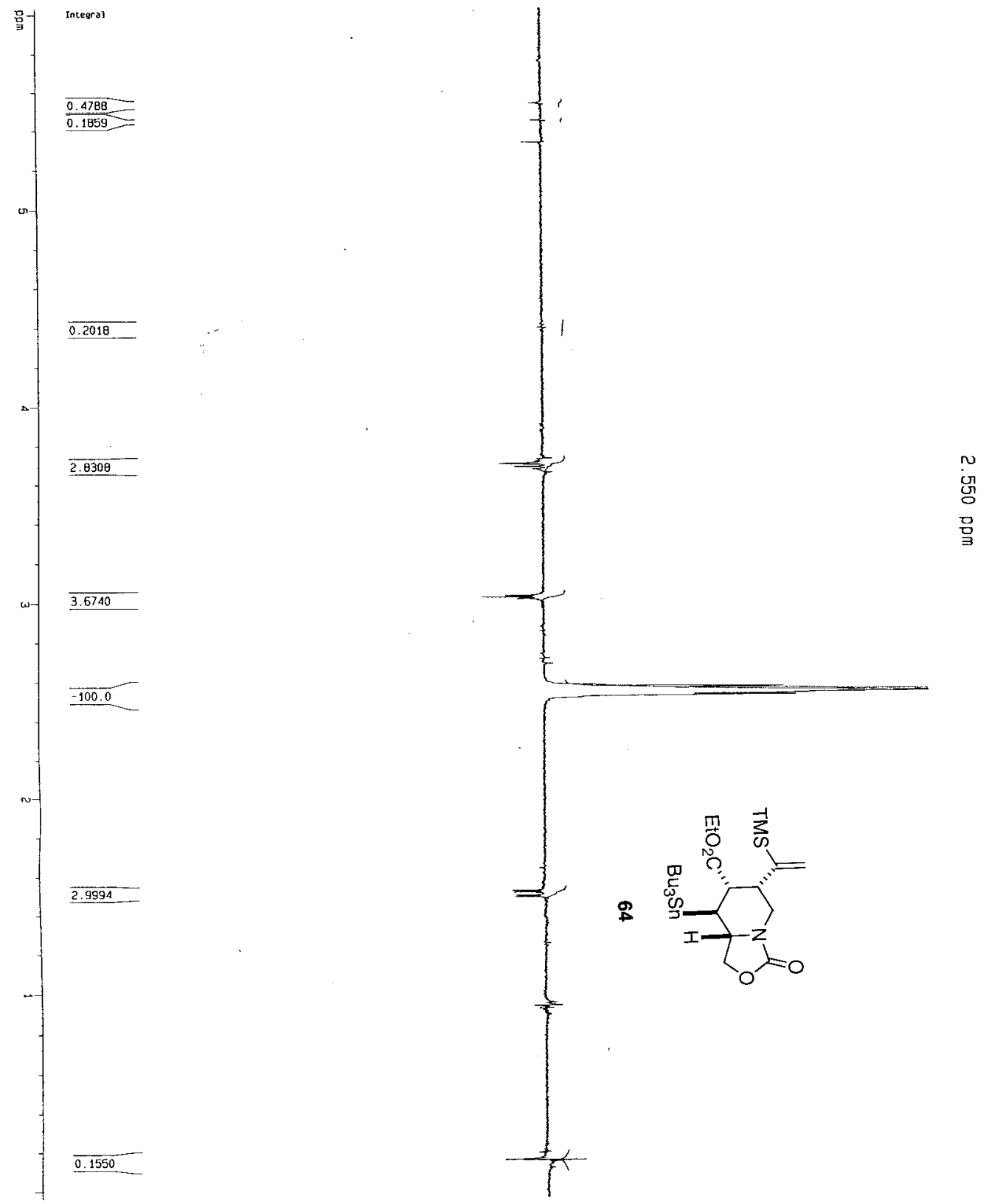

S- 